fon

WHC-MR- 0229

DE9 1007159

FEBO 71991

\title{
Status of Ground Water in the 1100 Area
}

\author{
A. G. Law \\ Date Published \\ December 1990
}

Prepared for the U.S. Department of Energy Office of Environmental Restoration and Waste Management

\footnotetext{
(2) Westinghouse Hanford Company Richland, Washington 99352

Hantord Operations and Engineering Contractor for the

U.S. Department of Energy under Contract DE-AC06-87RL10930
} 


\begin{abstract}
LEQAL DLSCLAMER
This report was prepared as an account of work sponsored by an agency of the United Statse Government. Noither the United States Government nor any agency thereof, nor any of their employees, nor any of their contractors, subcontractors or their omployeas, makes any warranty, express of impliod, or assumes any legal liability or responsibility for the accuracy, completeness, or any third party's use or the results of such use of any information, apparatus, product, or process disclosed, or represents that its use would not infringe privately owned rights. Reference herein to any specific commercial product, procese, of service by trade name. trademark, manufacturer, or otherwise, does not necessarily constitute or imply its endorsement, recommendation, or favoring by the United States Government or any agency thereof or its contractors or subcontrectors. The viewe and opinions of authors expressed herein do not necessarily state op reflect those of the United States Government or any agency thareol.
\end{abstract}

Princed in the Unilad Staine of Ameriea

DISCLW-2.CHP $(10-60)$ 
WHC-MR. 0229

8900604B R4

Attachment 1

Map of the 1100 Area Showing Location of Wells 


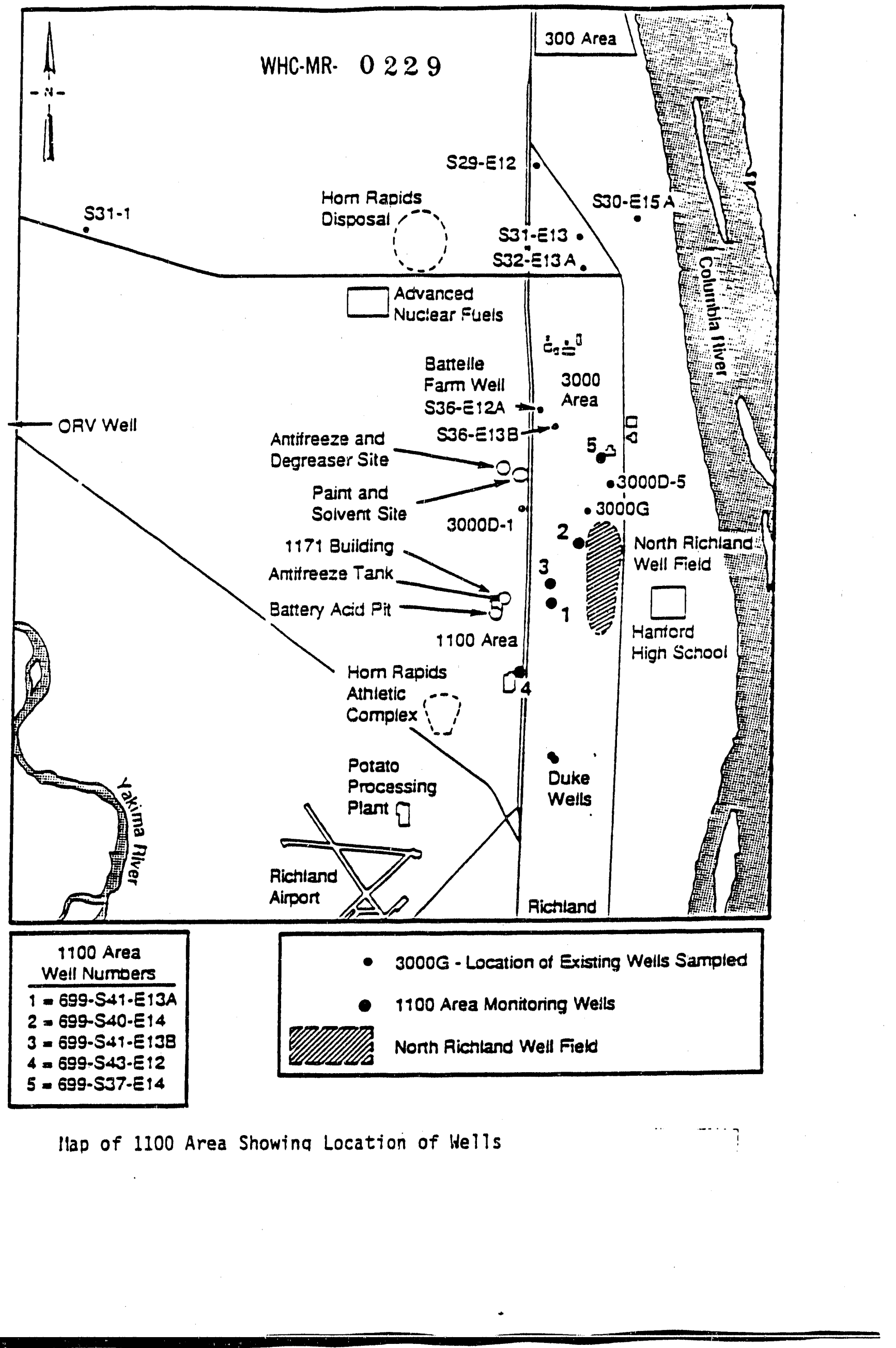


WHC-MR. 0229

$8900601 B$ R4

Attachment 2

\section{Results of Monthly Sampling of 1100 Area Wells}

The following table presents all of the results of monthly sampling and analyses between April 1989, and May 1990", for four constituents selected to be most indicative of the potential for contamination from U.S. Department of Energy facilities. The samples were collected from the three wells near the City of Richland well field. The detection limit and drinking water standards or maximum contaminant level are also listed in the table for each constituent.

* Sampling was discontinued for much of 1989 pending resolution of the purge water situation. 
WHC-MR- 0229

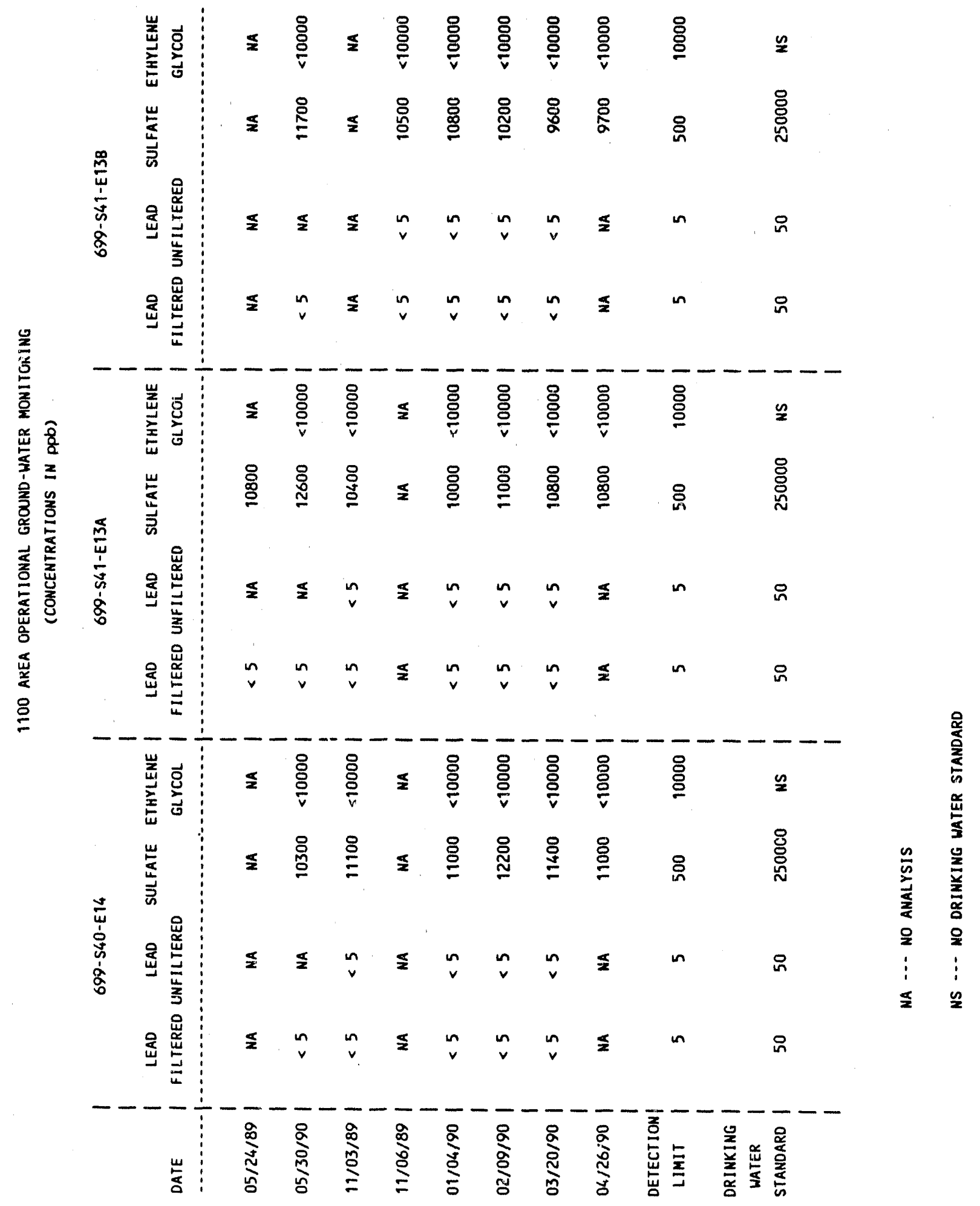


WHC-MR. 0229

$8900604 B$ R4

Attachment 3

Results for 1100 Area Ground-Water Monitoring

The following table presents a listing of the analytical results from sampling and analyses of five wells between April 1989, and May 1990* in the 1100 Area. The detection limit and drinking water standards or maximum contaminant level are also listed in the table for each constituent.

* Sampling was discontinued for much of 1989 pending resolution of the purge water situation. 
WHC-MR. 0229

$11 / 26 / 90$

Geosciences Group PARAOOX Database

Well Result Report

Page 1

1100 Area Groundwater Monitoring

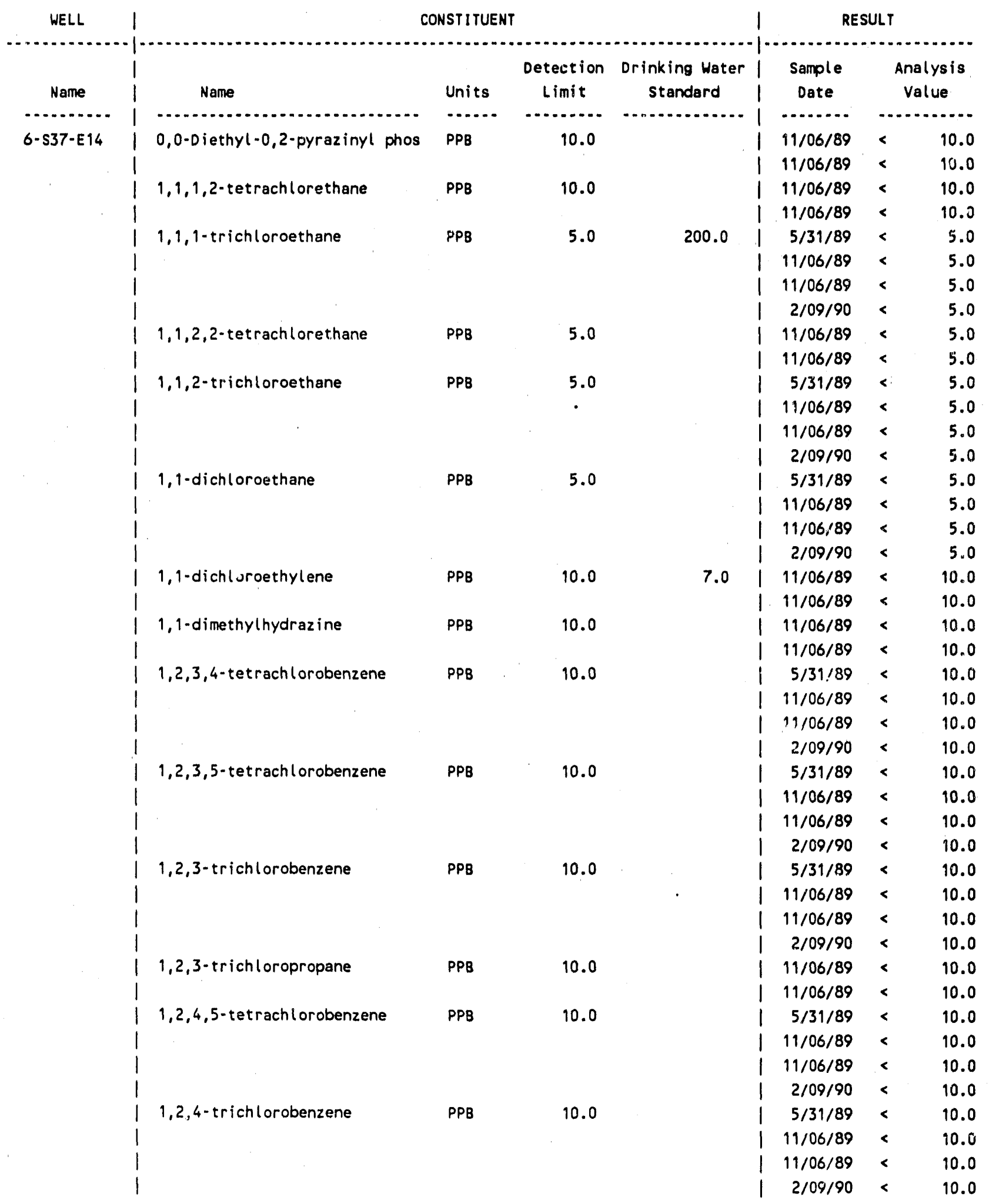




\section{WHC-MR- 0229}

$11 / 26 / 90$

Geosciences Group PARADOX Database

Well Result Report

1100 Area Groundwater Monitoring

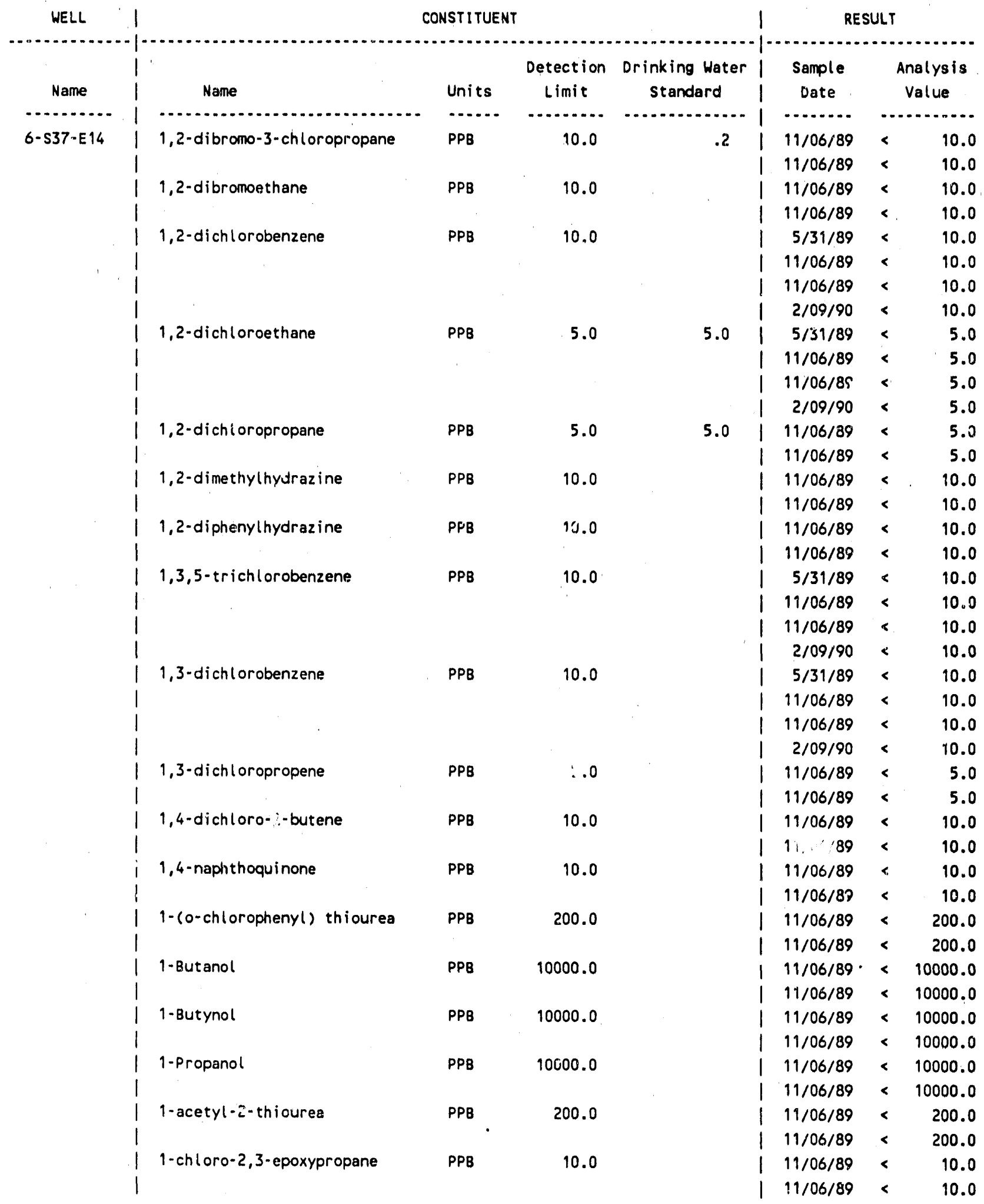


WHC-MR- 0229

$11 / 26 / 90$

Geosciences Group PARADOX Database

Well Result Report

Page 3

1100 Area Groundwater Monitoring

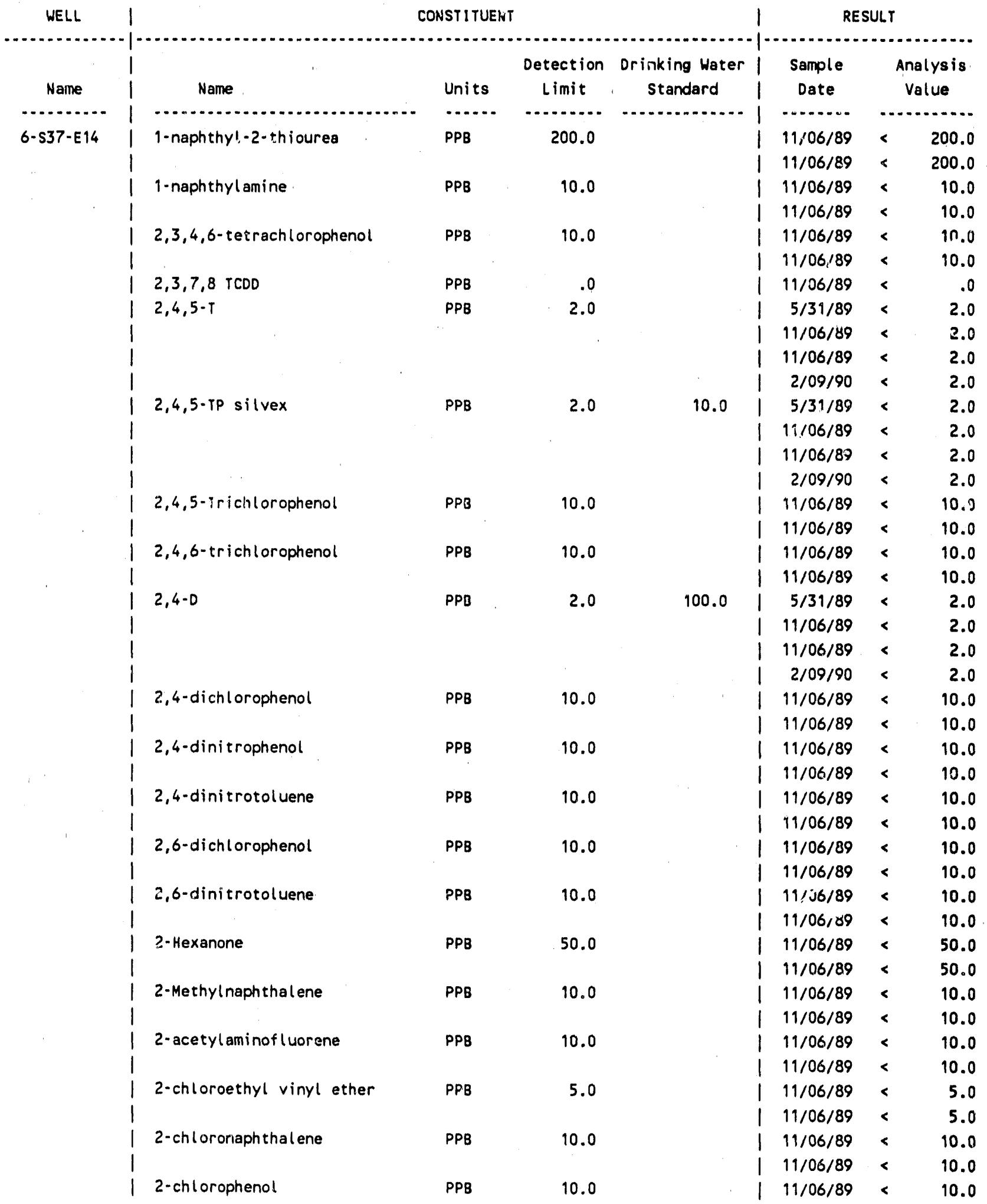


Well Result Report

1100 Area Groundwater Monitoring

\begin{tabular}{|c|c|c|c|c|c|c|c|c|}
\hline WELL & 1 & \multicolumn{4}{|c|}{ CONSTI IUENT } & \multicolumn{3}{|c|}{ RESULT } \\
\hline Name & 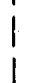 & Name & Units & $\begin{array}{l}\text { Detection } \\
\text { Limit }\end{array}$ & $\begin{array}{c}\text { Drinking Water } \\
\text { Standard }\end{array}$ & $\begin{array}{l}\text { Sample } \\
\text { Date }\end{array}$ & & $\begin{array}{l}\text { nalysis } \\
\text { Value }\end{array}$ \\
\hline ( & i & - & $\ldots . .$. & ............... & 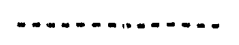 & $\ldots . . . . . .$. & $\cdots$ & .......... \\
\hline \multirow[t]{13}{*}{$6-537-E 14$} & i & 2-chtorophenol & PPB & 10.0 & & $11 / 06 / 89$ & $<$ & 10.0 \\
\hline & 1 & 2-cyclohexyl-4,6-dinitrophenol & PPB & 10.0 & & $11 / 06 / 89$ & $<$ & 10.0 \\
\hline & $i$ & & & & & $11 / 06 / 89$ & $<$ & 10.0 \\
\hline & i & 2-methyl-2-(methylthio) propio & PPB & 10.0 & & $11 / 06 / 89$ & $<$ & 10.0 \\
\hline & i & & & & & $11 / 06 / 89$ & $<$ & 10.0 \\
\hline & i & 2-methylaziridine & PPB & 10.0 & & $11 / 06 / 89$ & $<$ & 10.0 \\
\hline & 1 & & & & & $11 / 06 / 89$ & $<$ & 10.0 \\
\hline & 1 & 2-methyl lactonitrile & PPB & 10.0 & & $11 / 06 / 89$ & $<$ & 10.0 \\
\hline & i & & & & & $11 / 06 / 89$ & $<$ & 10.0 \\
\hline & i & 2-naph thylamine & PPB & 10.0 & & $11 / 06 / 89$ & $<$ & 10.0 \\
\hline & i & & & & & | 11/06/89 & $<$ & 10.0 \\
\hline & i & 2-picol ine & PPB & 10.0 & & $11 / 06 / 89$ & $<$ & 10.0 \\
\hline & i & & & & & | $11 / 06 / 89$ & $<$ & 10.0 \\
\hline \multirow[t]{31}{*}{ i } & $i$ & 2-propyn-1-ol & PPB & 10000.0 & & | $11 / 06 / 89$ & $<$ & 10000.0 \\
\hline & 1 & & & & & I 11/06/89 & $<$ & 10000.0 \\
\hline & 1 & 2-sec-butyl-4,6-dinitrophenol & PPB & 10.0 & & $111 / 06 / 89$ & $<$ & 10.0 \\
\hline & 1 & & & & & | 11/06/89 & $<$ & 10.0 \\
\hline & 1 & 3,3'-Dichlorobenzidine & PPB & 10.0 & & | $11 / 06 / 89$ & $<$ & 10.0 \\
\hline & 1 & & & & & $111 / 06 / 89$ & $<$ & 10.0 \\
\hline & 1 & 3,3'-dimethoxybenzidine & PPB & 10.0 & & I $11 / 06 / 89$ & $<$ & 10.0 \\
\hline & I & & & & & $111 / 06 / 89$ & $<$ & 10.0 \\
\hline & 1 & 3,3'-dimethylbenzidine & PPB & 10.0 & & | $1 i / 06 / 89$ & $<$ & 10.0 \\
\hline & 1 & & & & & I $11 / 06 / 89$ & $<$ & 10.0 \\
\hline & 1 & 3-chloropropionitrile & PPB & 10000.0 & & | $111 / 06 / 89$ & $<$ & 10000.0 \\
\hline & 1 & & & & & $11 / 06 / 89$ & $<$ & 10000.0 \\
\hline & 1 & 3-methylcholanthrene & PPB & 90.0 & & $111 / 06 / 89$ & $<$ & 10.0 \\
\hline & 1 & & & & & $111 / 06 / 89$ & $<$ & 10.0 \\
\hline & 1 & $4,4^{\prime}-$ methylenebis ( 2 -chloroanil & PPB & 10.0 & & | $11 / 06 / 89$ & $<$ & 10.0 \\
\hline & 1 & & & & & $111 / 06 / 89$ & $<$ & 10.0 \\
\hline & 1 & 4,6-dinitro-0-cresol and salts & PPB & 10.0 & & $111 / 06 / 89$ & $<$ & 10.0 \\
\hline & I & & & & & $111 / 06 / 89$ & $<$ & 10.0 \\
\hline & 1 & 4-Nitroquinol ine 1-oxide & PPB & 10.0 & & $111 / 06 / 89$ & $<$ & 10.0 \\
\hline & 1 & & & & & $111 / 06 / 89$ & $<$ & 10.0 \\
\hline & I & 4-aminobyphenyl & PPB & 10.0 & & I 11/06/89 & $<$ & 10.0 \\
\hline & 1 & & & & & | $11 / 06 / 89$ & $<$ & 10.0 \\
\hline & 1 & 4-bromophenyl phenyl ether & PPB & 10.0 & & $11 / 06 / 89$ & $<$ & 10.0 \\
\hline & 1 & & & & & $11 / 06 / 89$ & $<$ & 10.0 \\
\hline & 1 & 5-(aminomethyl)-3-isoxazol ol & PPB & 10.0 & & | 11/06/89 & $<$ & 10.0 \\
\hline & 1 & & & & & $111 / 06 / 80$ & $<$ & 10.0 \\
\hline & 1 & 5-nitro-o-toluidine & PPB & 10.0 & & $111 / 06 / 89$ & $<$ & 10.0 \\
\hline & 1 & & & & & $111 / 06 / 89$ & $<$ & 10.0 \\
\hline & 1 & 7,12 -dimethylbenz [a] anthracene & PPB & 10.0 & & $111 / 06 / 89$ & $<$ & 10.0 \\
\hline & 1 & & & & & $111 / 06 / 89$ & s & 10.0 \\
\hline & 1 & $7 H$-dibenzo $[C, g]$ carbazole & PPB & 10.0 & & $11 / 06 / 89$ & $<$ & 10.0 \\
\hline
\end{tabular}




\section{WHC-MR- 0229}

Geosciences Group PARADOX Database

Well Result Report

1100 Area Groundwater Monitoring

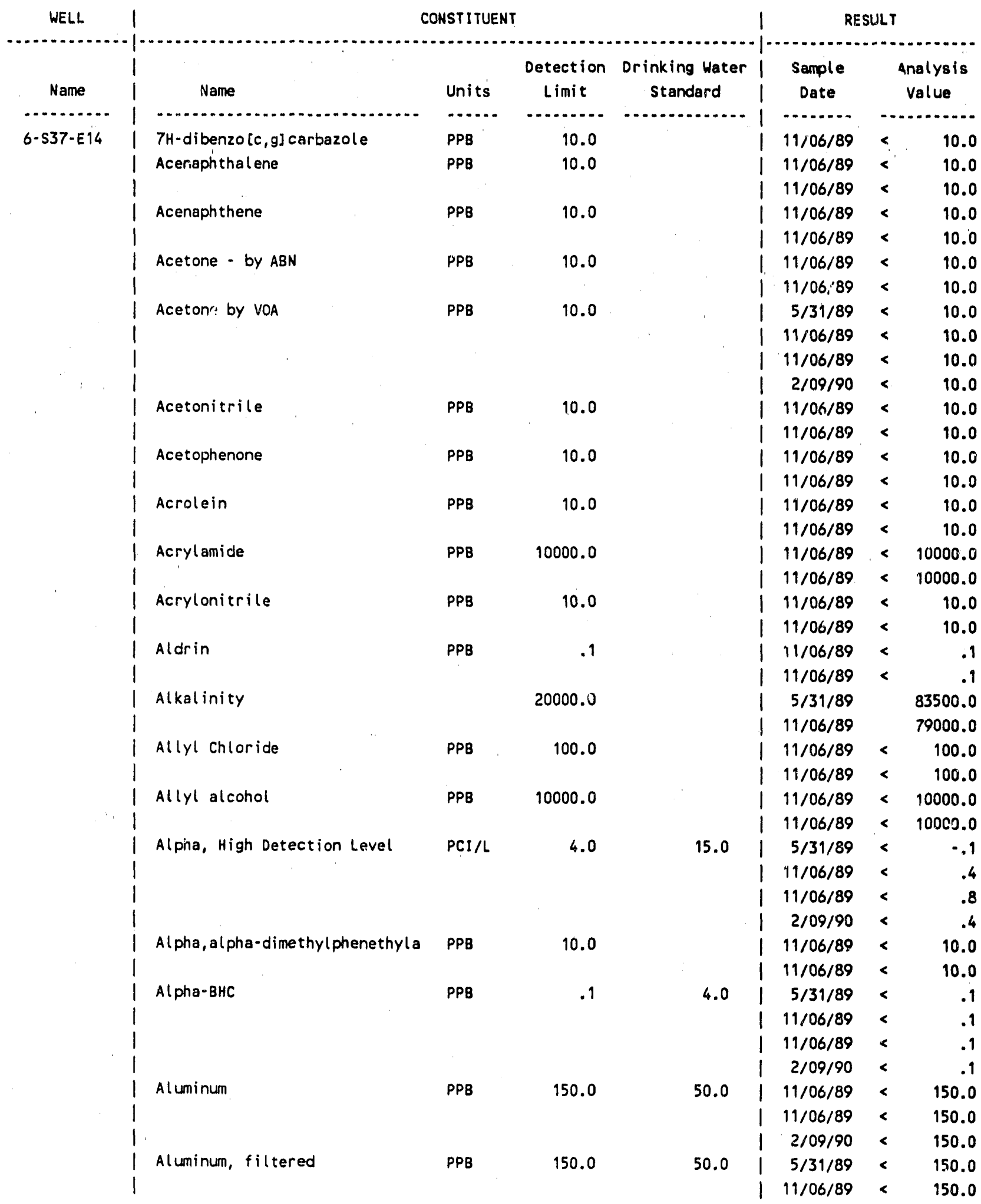


WHC-MR- 0229

$11 / 26 / 90$

Geosciences Group PARADOX Database

Hell Result Report

1100 Area Groundwater Monitoring

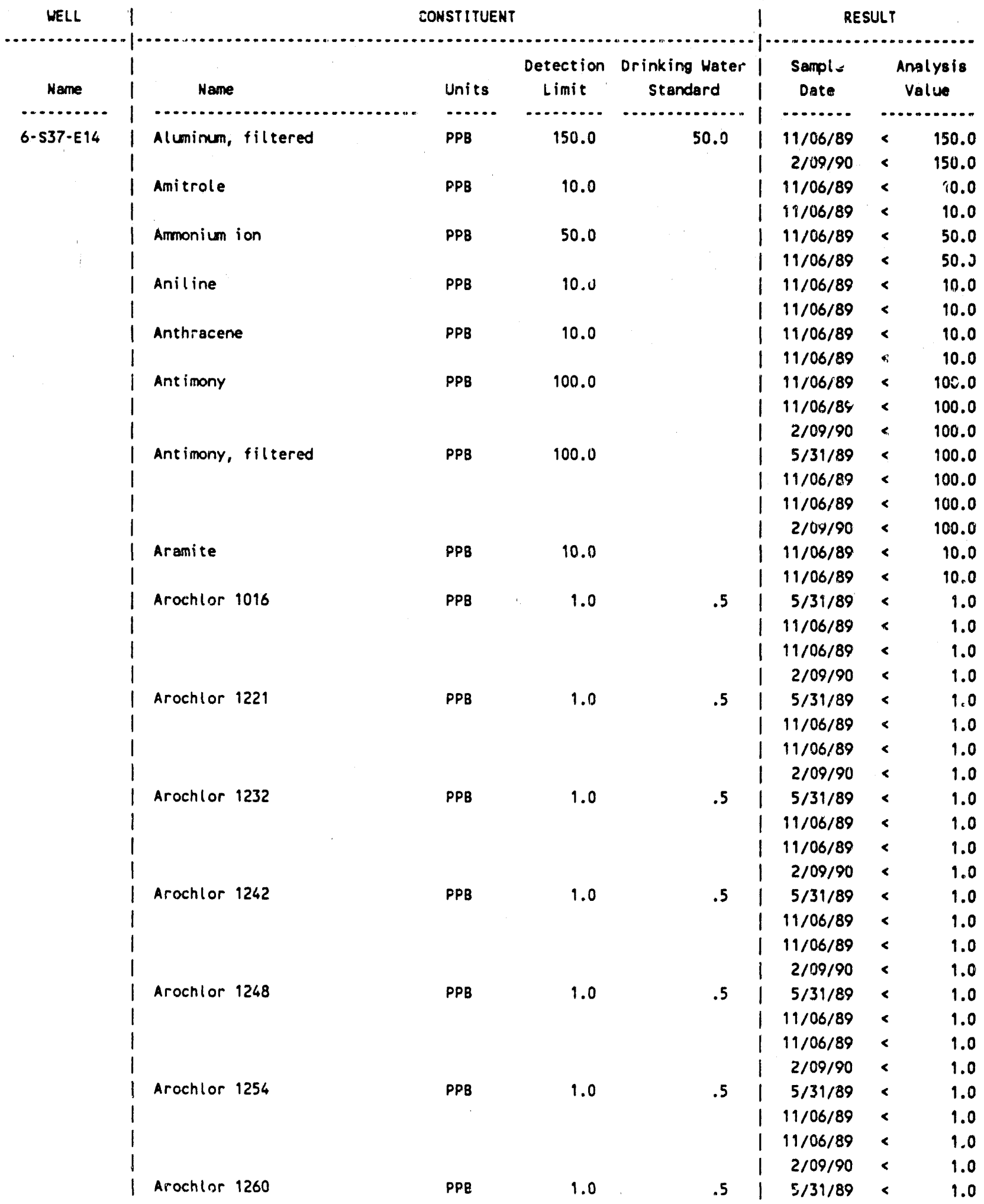




\section{WHC-MR. 0229}

$11 / 26 / 90$

Geosciences Group PARADOX Database

Well Result Report

i100 Area Groundwater Monitoring

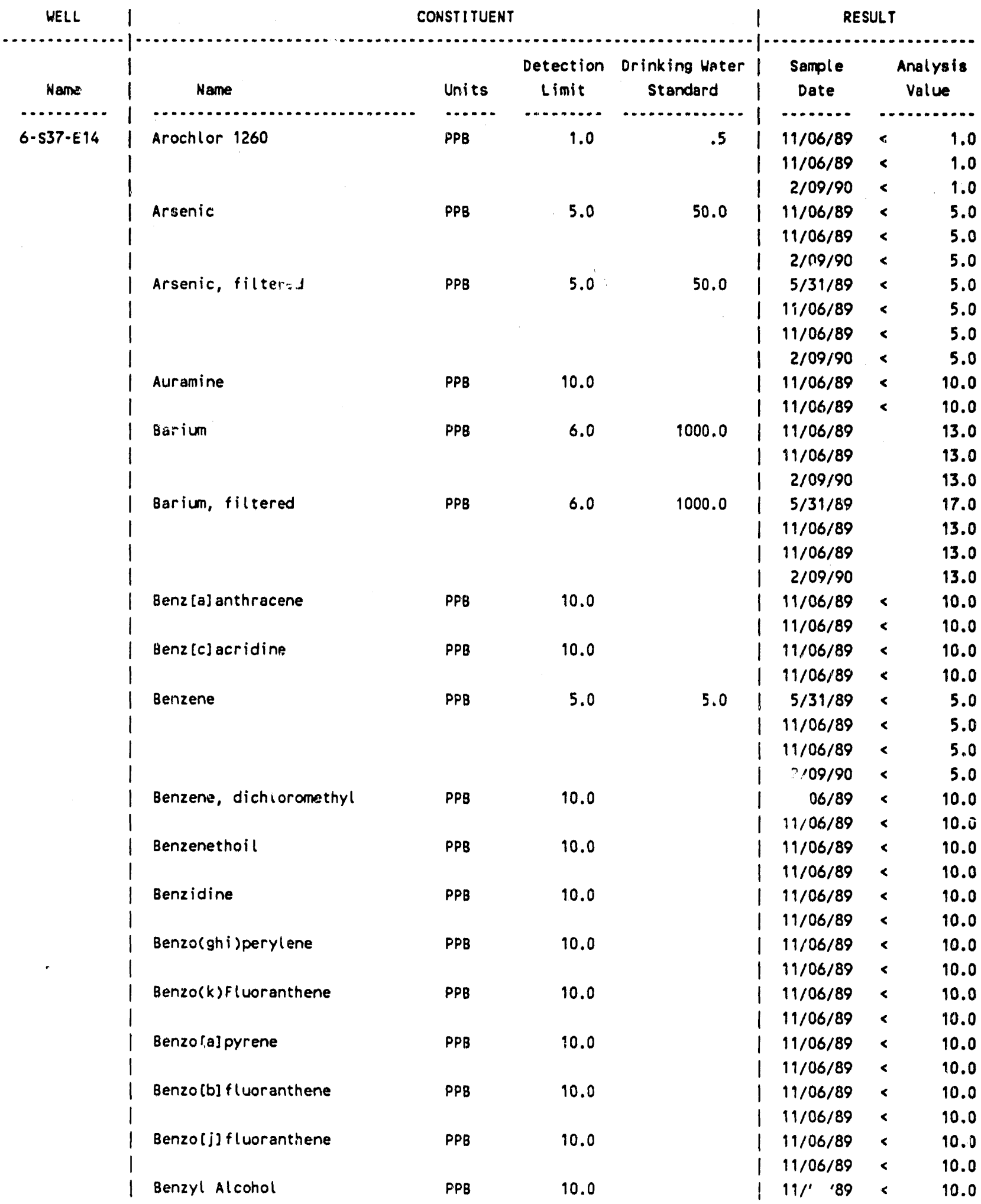


WHC-MR- 0229

$11 / 26 / 90$

Geosciences Group PARADOX Database

Well Result Report

1100 Area Groundwater Monitoring

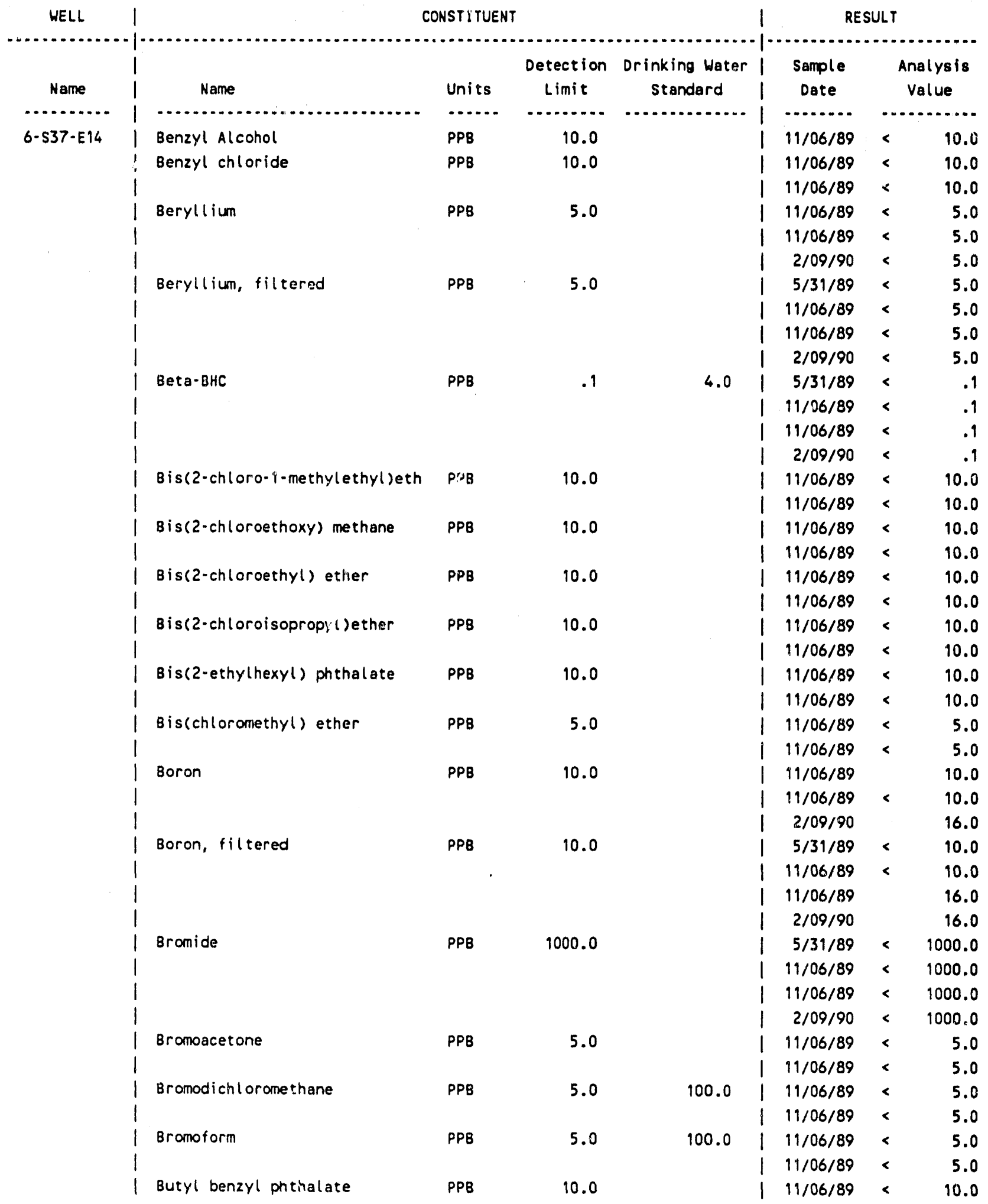




\section{IWHC-MR. 0229}

Well Result Report

1100 Area Groundwater Monitoring

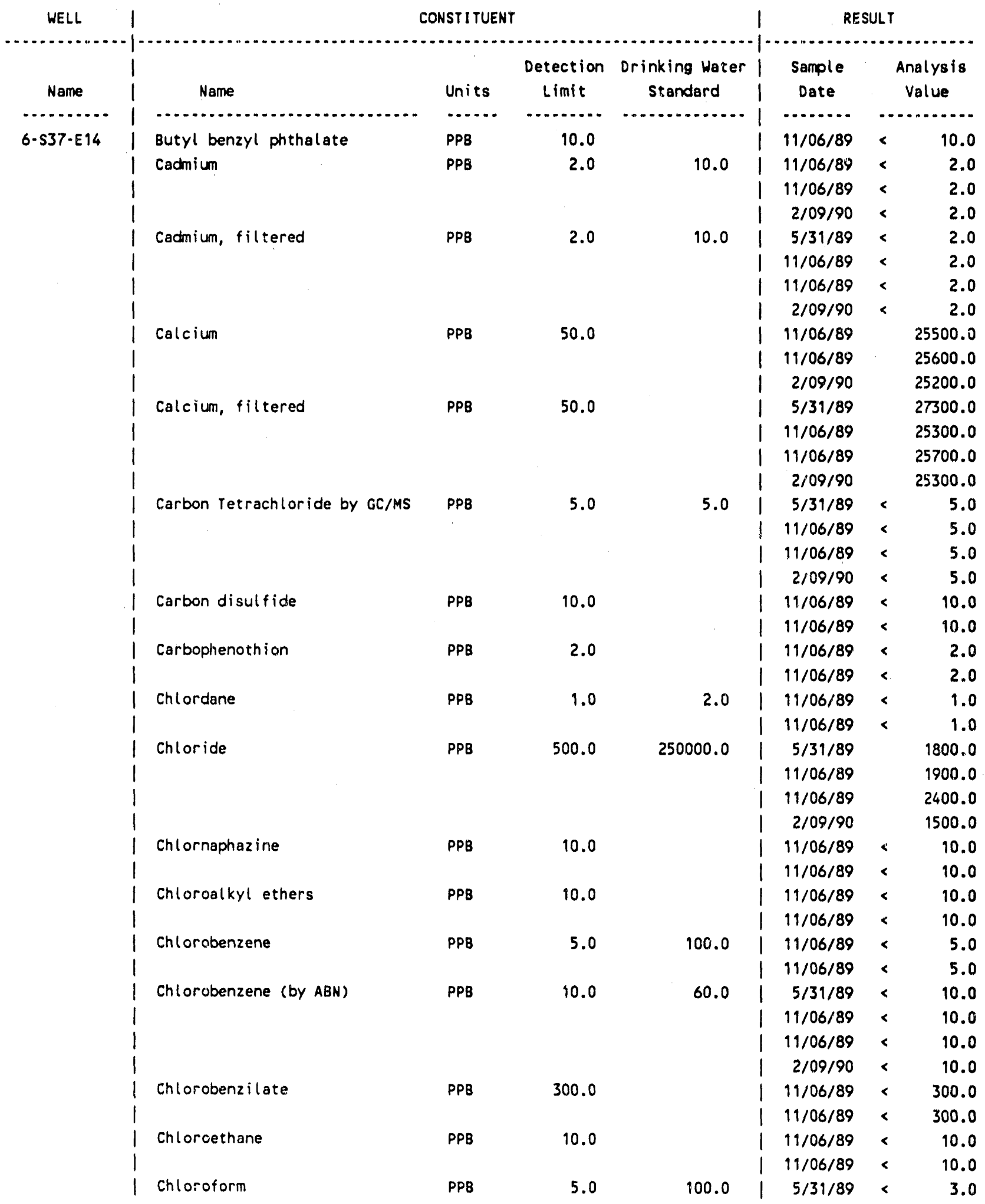


WHC-MR. 0229

Geosciences Group PARADOX Database

Well Result Report

1100 Area Groundwater Monitoring

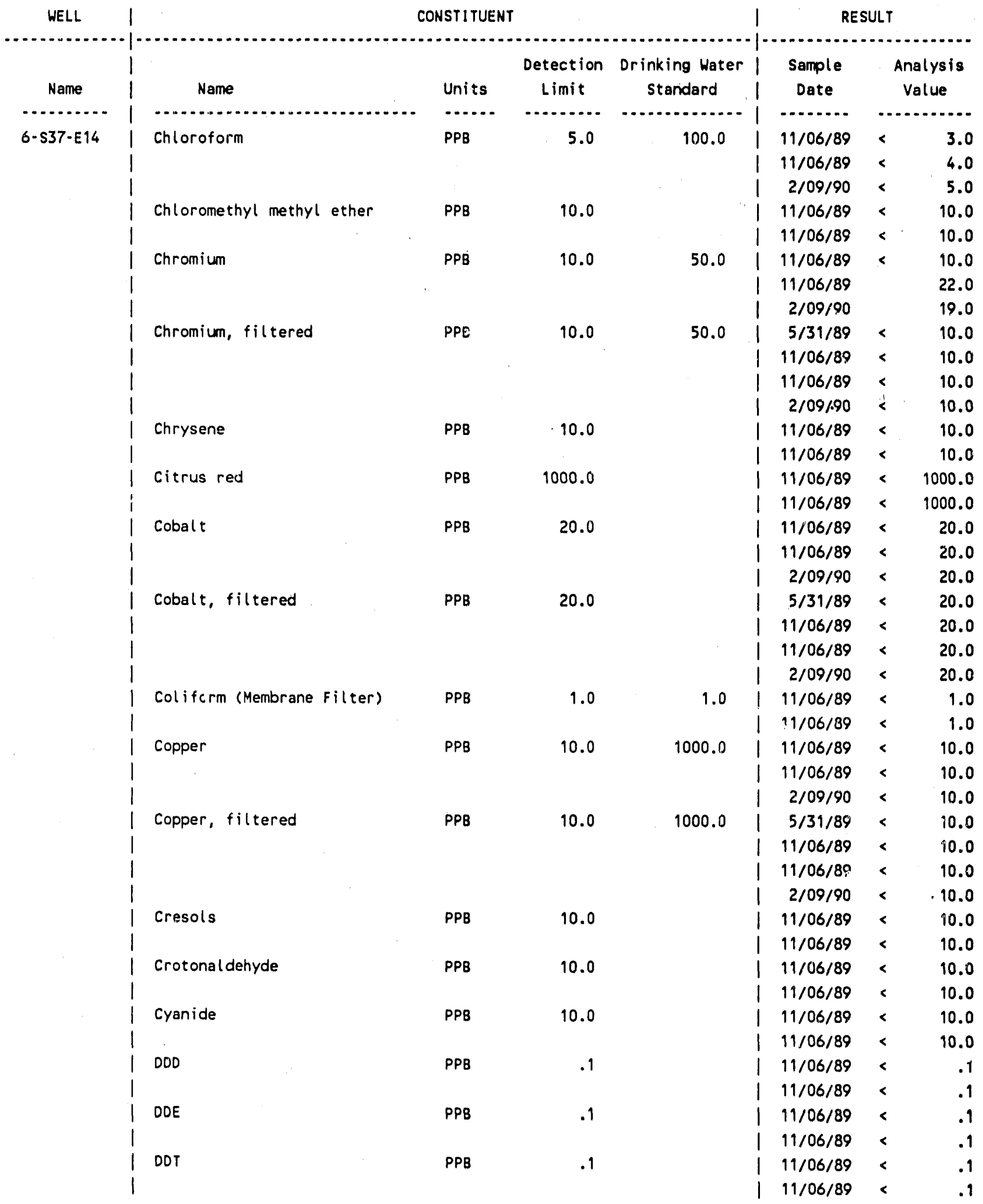


WHC-MR- 0229

$11 / 26 / 90$

Geosciences Group PARADOX Database

Well Result Report

Page 11

1100 Area Groundwater Monitoring

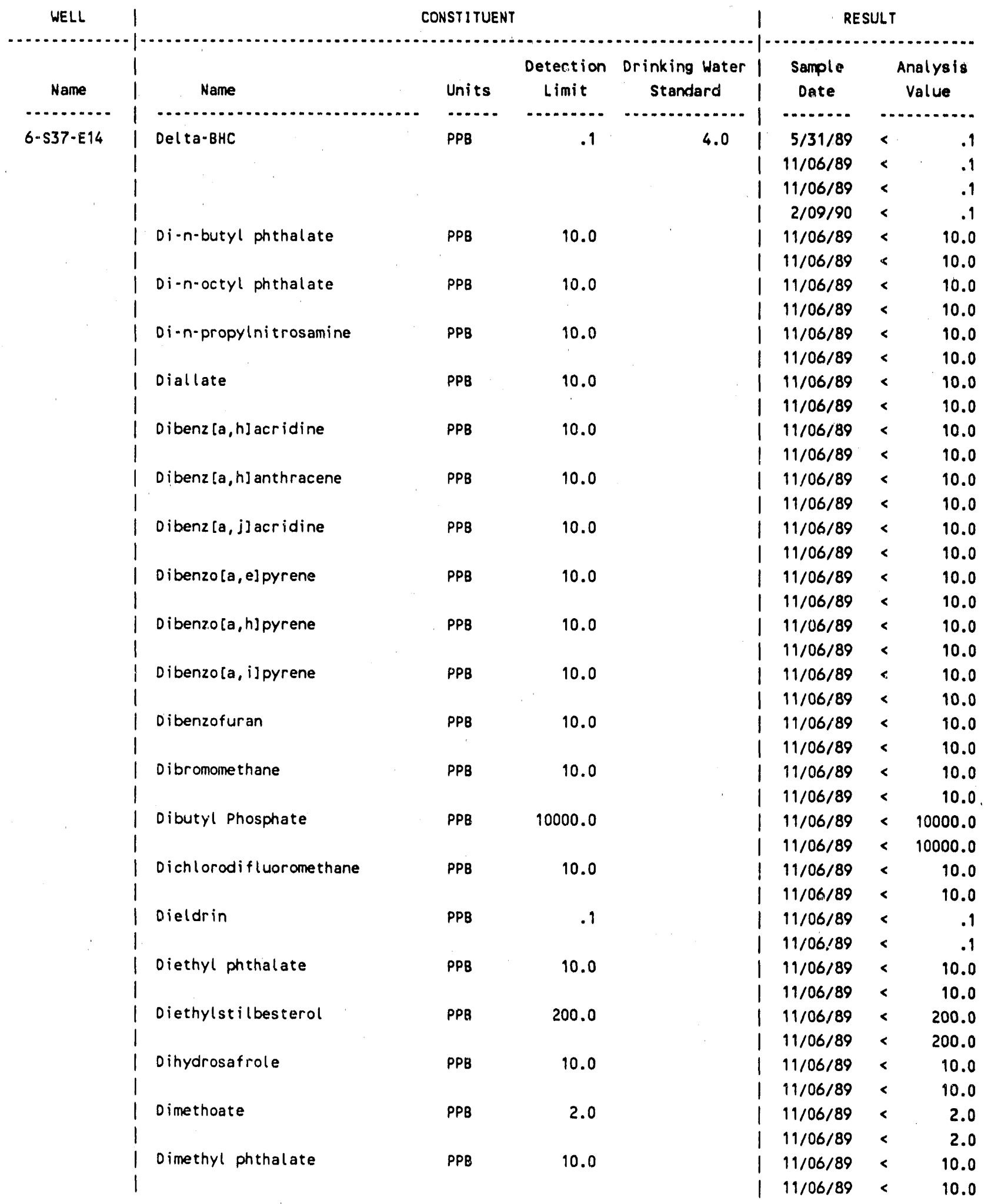




\section{WHC-MR. 0229}

Well Result Report

1100 Area Groundwater Monitoring

\begin{tabular}{|c|c|c|c|c|c|c|c|c|}
\hline WELL & 1 & & NSTITUEN & & & RE & & \\
\hline Name & 1 & Name & Units & $\begin{array}{l}\text { Detection } \\
\text { Limit }\end{array}$ & $\begin{array}{c}\text { Orinking Water } \\
\text { standard }\end{array}$ & $\left\{\begin{array}{c}\text { Sample } \\
\text { Date }\end{array}\right.$ & & $\begin{array}{l}\text { inalysis } \\
\text { Value }\end{array}$ \\
\hline$\ldots \ldots \ldots$ & 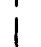 & - & $\ldots .$. & $\ldots \ldots$ & $\ldots \ldots \ldots \ldots$ & $\ldots \ldots$ & & .......... \\
\hline 6-S37-E14 & $i$ & Dinitrobenzene & PPB & 10.0 & & $11 / 06 / 89$ & $<$ & 10.0 \\
\hline & i & & & & & $11 / 06 / 89$ & $<$ & 10.0 \\
\hline & i & Dinoseb & PPB & 10.0 & & $11 / 06 / 89$ & $<$ & 10.0 \\
\hline & i & & & & & $11 / 06 / 89$ & $<$ & 10.0 \\
\hline & i & Dioxane & PPB & 500.0 & & $11 / 06 / 89$ & $<$ & 500.0 \\
\hline & i & & & & & $11 / 06 / 89$ & $<$ & 500.0 \\
\hline & i & Diphenylamine & PPB & 10.0 & & $11 / 06 / 89$ & $<$ & 10.0 \\
\hline & i & & & & & $11 / 06 / 89$ & $<$ & 10.0 \\
\hline & i & Disulfoton & PPB & 2.0 & & $11 / 06 / 89$ & $<$ & 2.0 \\
\hline & i & & & & & $11 / 06 / 89$ & $<$ & 2.0 \\
\hline & i & Endosulfan 1 & PPB & .1 & & $11 / 06 / 89$ & $<$ & .1 \\
\hline & i & & & & & $11 / 06 / 89$ & $<$ & .1 \\
\hline & I & Endosulfan 11 & PPB & .1 & & $11 / 06 / 89$ & $<$ & .1 \\
\hline & $i$ & & & & & $11 / 06 / 89$ & $<$ & .1 \\
\hline & i & Endosulfan sulfate & PPB & .5 & & $11 / 06 / 89$ & $<$ & .5 \\
\hline & i & & & & & $11 / 06 / 89$ & $<$ & .5 \\
\hline & i & Endrin & PPB & .1 & .2 & $5 / 31 / 89$ & $<$ & .1 \\
\hline & i & & & & & $11 / 06 / 89$ & $<$ & .1 \\
\hline & 1 & & & & & $11 / 06 / 89$ & $<$ & .1 \\
\hline & 1 & & & & & $2 . / 09 / 90$ & $<$ & .1 \\
\hline & 1 & Ethanol & PPB & 10000.0 & & $11 / 06 / 89$ & $<$ & 10000.0 \\
\hline & 1 & & & & & $11 / 06 / 89$ & $<$ & 10000.0 \\
\hline & i & Ethyl benzene & PPB & 5.0 & 700.0 & $11 / 06 / 89$ & $<$ & 5.0 \\
\hline & 1 & & & & & $11 / 06 / 89$ & $<$ & 5.0 \\
\hline & 1 & Ethyl carbamate & PPB & 10000.0 & & $11 / 06 / 89$ & $<$ & 10000.0 \\
\hline & 1 & & & & & $11 / 06 / 89$ & $<$ & 10000.0 \\
\hline & 1 & Ethyl cyanide & PPB & 10000.0 & & | $11 / 06 / 89$ & $<$ & 10000.0 \\
\hline & i & & & & & $11 / 06 / 89$ & $<$ & 10000.0 \\
\hline & i & Ethyl methacrylate & PPB & 10.0 & & $11 / 06 / 89$ & $<$ & 10.0 \\
\hline & 1 & & & & & $11 / 05 / 89$ & $<$ & 10.0 \\
\hline & i & Ethyl methanesulfonate & PPB & 10.0 & & $11 / 06 / 89$ & $<$ & 10.0 \\
\hline & 1 & & & & & $11 / 06 / 89$ & $<$ & 10.0 \\
\hline & 1 & Ethylene Glycol & PPB & 10000.0 & & i $11 / 06 / 89$ & $<$ & 10000.0 \\
\hline & 1 & & & & & $11 / 06 / 89$ & 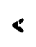 & 10000.0 \\
\hline & 1 & Ethylene glycol & PPB & 10000.0 & & $15 / 31 / 89$ & $<$ & 10000.0 \\
\hline & i & & & & & $2 / 09 / 90$ & $<$ & 10000.0 \\
\hline & 1 & Ethylene oxide & PPB & 10.0 & & | $11 / 06 / 89$ & $<$ & 10.0 \\
\hline & i & & & & & $11 / 06 / 89$ & $<$ & 10.0 \\
\hline & 1 & Ethyleneimine & PPB & 10.0 & & $111 / 06 / 89$ & $<$ & 10.0 \\
\hline & 1 & & & & & $111 / 06 / 89$ & $<$ & 10.0 \\
\hline & 1 & Ethylenethiourea & PFB & 200.0 & & | $11 / 06 / 89$ & $<$ & 200.0 \\
\hline & 1 & & & & & $11 / 06 / 89$ & $<$ & 200.0 \\
\hline & i & Fluoranthene & PPB & 10.0 & & $11 / 06 / 89$ & $<$ & 10.0 \\
\hline & 1 & & & & & $11 / 06 / 89$ & $<$ & 10.0 \\
\hline
\end{tabular}




\section{WHC-MR- 0229}

Geosciences Group PARADOX Database

Well Result Report

1100 Area Groundwater Monitoring

\begin{tabular}{|c|c|c|c|c|c|c|c|c|c|}
\hline WELL & \multicolumn{5}{|c|}{ CONST I TUENT } & 1 & \multicolumn{3}{|c|}{ RESUI.T } \\
\hline Name & 1 & Name & Units & $\begin{array}{l}\text { Detection } \\
\text { Limit }\end{array}$ & $\begin{array}{l}\text { Drinking Water } \\
\text { standard }\end{array}$ & 1 & $\begin{array}{l}\text { Sample } \\
\text { Date }\end{array}$ & & alys is \\
\hline ….... & 1 & - & -..... & ............ & n & 1 & ......... & & \\
\hline \multirow[t]{44}{*}{ 6-S37-E 14} & 1 & Fluorene & PPB & 10.0 & & 1 & $11 / 06 / 89$ & $<$ & 10.0 \\
\hline & 1 & & & & & 1 & $11 / 06 / 89$ & $<$ & 10.0 \\
\hline & 1 & Fluoride & PPB & 500.0 & 4000.0 & 1 & $5 / 31 / 89$ & $<$ & 500.0 \\
\hline & i & & & & & i & $11 / 06 / 89$ & $<$ & 500.0 \\
\hline & i & & & & & i & $11 / 06 / 89$ & $<$ & 500.0 \\
\hline & 1 & & & & & 1 & $2 / 09 / 90$ & $<$ & 500.0 \\
\hline & 1 & Formal in & PPB & 500.0 & & 1 & $11 / 06 / 89$ & $<$ & 500.0 \\
\hline & 1 & & & & & 1 & $11 / 06 / 89$ & $<$ & 500.0 \\
\hline & 1 & Eamma-BHC & PPB & .1 & 4.0 & i & $5 / 31 / 89$ & $<$ & .1 \\
\hline & 1 & & & & & 1 & $11 / 06 / 89$ & $<$ & .1 \\
\hline & 1 & & & & & 1 & $11 / 06 / 89$ & $<$ & .1 \\
\hline & 1 & & & & & 1 & $2 / 09 / 90$ & $<$ & .1 \\
\hline & 1 & Gross beta & $\mathrm{PCI} / \mathrm{L}$ & 8.0 & 50.0 & 1 & $5 / 31 / 89$ & & 2.6 \\
\hline & 1 & & & & & i & $11 / 06 / 89$ & $<$ & 1.3 \\
\hline & 1 & & & & & 1 & $11 / 06 / 89$ & & 4.7 \\
\hline & 1 & & & & & 1 & $2 / 09 / 90$ & & 4.0 \\
\hline & 1 & Heptachlor & PPB & .1 & .4 & I & $11 / 06 / 89$ & $<$ & .1 \\
\hline & 1 & & & & & 1 & $11 / 06 / 89$ & $<$ & .1 \\
\hline & 1 & Heptchlor epoxide & PPB & .1 & & i & $11 / 06 / 89$ & $<$ & .1 \\
\hline & 1 & & & & & i & $11 / 06 / 89$ & $<$ & .1 \\
\hline & 1 & Hexachlorobenzene & PPB & 10.0 & & i & $5 / 31 / 89$ & $<$ & 10.0 \\
\hline & 1 & & & & & 1 & $11 / 06 / 89$ & $<$ & 10.0 \\
\hline & 1 & & & & & 1 & $11 / 06 / 89$ & $<$ & 10.0 \\
\hline & 1 & & & & & i & $2 / 09 / 90$ & $<$ & 10.0 \\
\hline & 1 & Hexachlor obutadiene & PPB & 10.0 & & 1 & $11 / 06 / 89$ & $<$ & 10.0 \\
\hline & 1 & & & & & i & $11 / 06 / 89$ & $<$ & 10.0 \\
\hline & 1 & Hexachlorocycl opentadi ene & PPB & 10.0 & & 1 & $11 / 06 / 89$ & $<$ & 10.0 \\
\hline & 1 & & & & & i & $11 / 06 / 89$ & s & 10.0 \\
\hline & 1 & Hexachloroethane & PPB & 10.0 & & i & $11 / 06 / 89$ & $<$ & 10.0 \\
\hline & 1 & & & & & 1 & $11 / 06 / 89$ & $<$ & 10.0 \\
\hline & $i$ & Hexachlo: ophene & PPB & 10.0 & & 1 & $5 / 31 / 89$ & $<$ & 10.0 \\
\hline & 1 & & & & & i & $11 / 06 / 89$ & $<$ & 10.0 \\
\hline & 1 & & & & & 1 & $11 / 06 / 89$ & $<$ & 10.0 \\
\hline & 1 & & & & & 1 & $2 / 09 / 90$ & $<$ & 10.0 \\
\hline & 1 & Hexachloropropene & PPB & 10.0 & & 1 & $11 / 06 / 89$ & $<$ & 10.0 \\
\hline & 1 & & & & & 1 & $11 / 06 / 89$ & $<$ & 10.0 \\
\hline & 1 & Hydrazine, Low Detection Level & PPB & 30.0 & & 1 & $11 / 06 / 89$ & $<$ & 30.0 \\
\hline & I & & & & & 1 & $11 / 06 / 89$ & $<$ & 30.0 \\
\hline & 1 & Indeno(1,2,3-cd)pyrene & PPB & 10.0 & & 1 & $11 / 06 / 89$ & $<$ & 10.0 \\
\hline & 1 & & & & & 1 & $11 / 06 / 89$ & $<$ & 10.0 \\
\hline & 1 & lodome thane & PPB & 10.0 & & 1 & $11 / 06 / 89$ & $<$ & 10.0 \\
\hline & 1 & & & & & 1 & $11 / 06 / 89$ & $<$ & 10.0 \\
\hline & I & Iron & PPB & 30.0 & 300.0 & 1 & $11 / 06 / 89$ & & 101.0 \\
\hline & 1 & & & & & i & $11 / 06 / 89$ & & 103.0 \\
\hline
\end{tabular}




\section{WHC-MR. 0229}

$11 / 26 / 90$

Geosciences Group PARADOX Database

Well Result Report

1100 Area Groundwater Monitoring

\begin{tabular}{|c|c|c|c|c|c|c|c|}
\hline \multirow{2}{*}{$\begin{array}{l}\text { WELL } \\
\text { Name }\end{array}$} & \multicolumn{4}{|c|}{ CONSTI TUENT } & \multicolumn{3}{|c|}{ RESULT } \\
\hline & Name & Units & $\begin{array}{l}\text { Detection } \\
\text { Limit }\end{array}$ & $\begin{array}{c}\text { Drinking Water } \\
\text { Standard }\end{array}$ & $\begin{array}{l}\text { Sample } \\
\text { Date }\end{array}$ & & $\begin{array}{l}\text { Analysis } \\
\text { Value }\end{array}$ \\
\hline & | & $\cdots \ldots$ & -.......... & $\ldots+\ldots$ & 1 & & .......... \\
\hline \multirow[t]{44}{*}{$6-537-E 14$} & I Iron & PPB & 30.0 & 300.0 & i $2 / 09 / 90$ & & 114.0 \\
\hline & I Iron, filtered & PPB & 30.0 & 300.0 & $5 / 31 / 89$ & $<$ & 30.0 \\
\hline & i n n & & & & $11 / 06 / 89$ & $<$ & 30.0 \\
\hline & 1 & & & & I $11 / 06 / 89$ & $<$ & 30.0 \\
\hline & 1 & & & & $2 / 09 / 90$ & $<$ & 30.0 \\
\hline & I I sobutyl alcohol & PPB & 10000.0 & & 1 $11 / 06 / 89$ & $<$ & 10000.0 \\
\hline & 1 & & & & I 11/06/89 & $<$ & 10000.0 \\
\hline & I Isodrin & PPB & 10.0 & & I $11 / 06 / 89$ & $<$ & 10.0 \\
\hline & i & & & & i 11/06/89 & $<$ & 10.0 \\
\hline & I sophorone & PPB & 10.0 & & I $11 / 06 / 89$ & $<$ & 10.0 \\
\hline & 1 & & & & I $11 / 06 / 89$ & $<$ & 10.0 \\
\hline & I Isosafrole & PPB & 10.0 & & i $11 / 06 / 89$ & $<$ & 10.0 \\
\hline & 1 & & & & 1. $11 / 06 / 80$ & $<$ & 10.0 \\
\hline & I Kepone & PPB & 1.0 & & I 11/06/89 & $<$ & 1.0 \\
\hline & 1 & & & & $1 \quad 11 / 06 / 89$ & $<$ & 1.0 \\
\hline & I Kerosene & PPB & 10000.0 & & $15 / 31 / 89$ & $<$ & 10000.0 \\
\hline & i & & & & I 11/06/89 & $<$ & 10000.0 \\
\hline & 1 & & & & I 11/06/89 & $<$ & 10000.0 \\
\hline & 1 & & & & $12 / 09 / 90$ & $<$ & 10000.0 \\
\hline & | Lead (graphite furnace) & PPB & 5.0 & 50.0 & I $11 / 06 / 89$ & $<$ & 5.0 \\
\hline & 1 & & & & I 11/06/89 & $<$ & 5.0 \\
\hline & 1 & & & & $1 \quad 2 / 09 / 90$ & $<$ & 5.0 \\
\hline & I Lead, filtered & PPB & 5.0 & 50.0 & i $5 / 31 / 89$ & $<$ & 5.0 \\
\hline & 1 & & & & I 11/06/89 & $<$ & 5.0 \\
\hline & 1 & & & & | 11/06/89 & $<$ & 5.0 \\
\hline & 1 & & & & $12 / 09 / 90$ & $<$ & 5.0 \\
\hline & I Lithium & PPB & 10.0 & & I $11 / 06 / 89$ & $<$ & 10.0 \\
\hline & 1 & & & & I $11 / 06 / 89$ & $<$ & 10.0 \\
\hline & 1 & & & & $12 / 09 / 90$ & $<$ & 10.0 \\
\hline & I Lithium, filtered & PPB & 10.0 & & $5 / 31 / 89$ & $<$ & 10.0 \\
\hline & 1 & & & & I 11/06/89 & $<$ & 10.0 \\
\hline & 1 & & & & $111 / 06 / 89$ & $<$ & 10.0 \\
\hline & 1 & & & & $1 \quad 2 / 09 / 90$ & $<$ & 10.0 \\
\hline & I Magnesium & PPB & 50.0 & & I $11 / 06 / 89$ & & 4620.0 \\
\hline & 1 & & & & I 19/06/89 & & 4650.0 \\
\hline & 1 & & & & i $2 / 09 / 90$ & & 4530.0 \\
\hline & I Magnesium, filtered & PPB & 50.0 & & $5 / 31 / 89$ & & 5000.0 \\
\hline & 1 & & & & $11 / 06 / 89$ & & 4600.0 \\
\hline & 1 & & & & I 11/06/89 & & 4660.0 \\
\hline & 1 & & & & $1 \quad 2 / 09 / 90$ & & 4510.0 \\
\hline & I Maleic hydrizide & PPB & 500.0 & & I 11/06/89 & $<$ & 500.0 \\
\hline & 1 & & & & I 11/06/89 & $<$ & 500.0 \\
\hline & I Malononitrile & PPB & 10.0 & & I 11/06/89 & $<$ & 10.0 \\
\hline & 1 & & & & I 11/06/89 & $<$ & 10.0 \\
\hline
\end{tabular}




\section{WHC-MR. 0229}

$11 / 26 / 90$

Geosciences Group PARADOX Database

Well Result Report

1100 Area Groundwater Monitoring

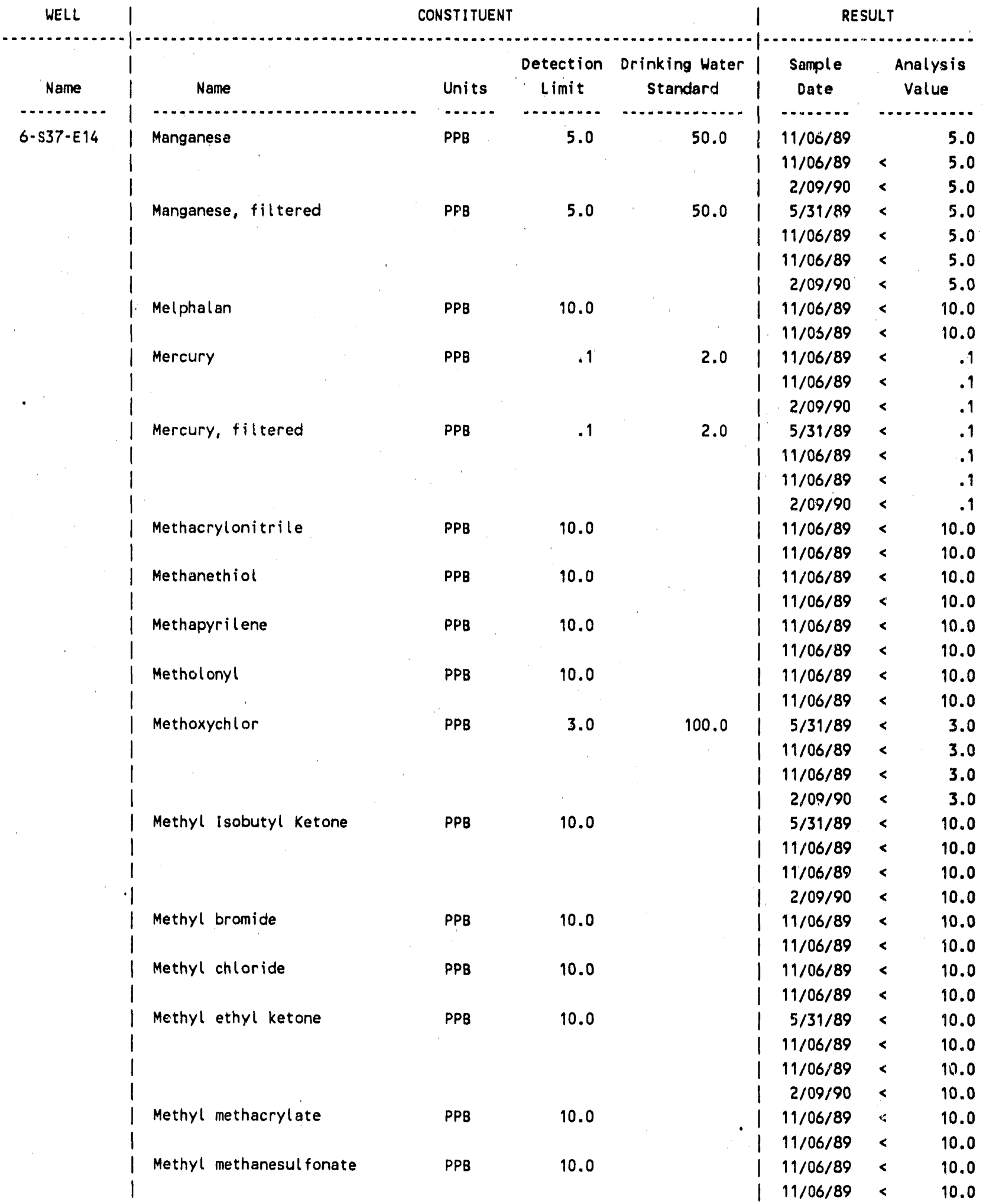




\section{WHC-MR- 0229}

$11 / 26 / 90$

Geosciences Group PARADOX Database

Well Result Report

Page 16

1100 Area Groundwater Monitoring

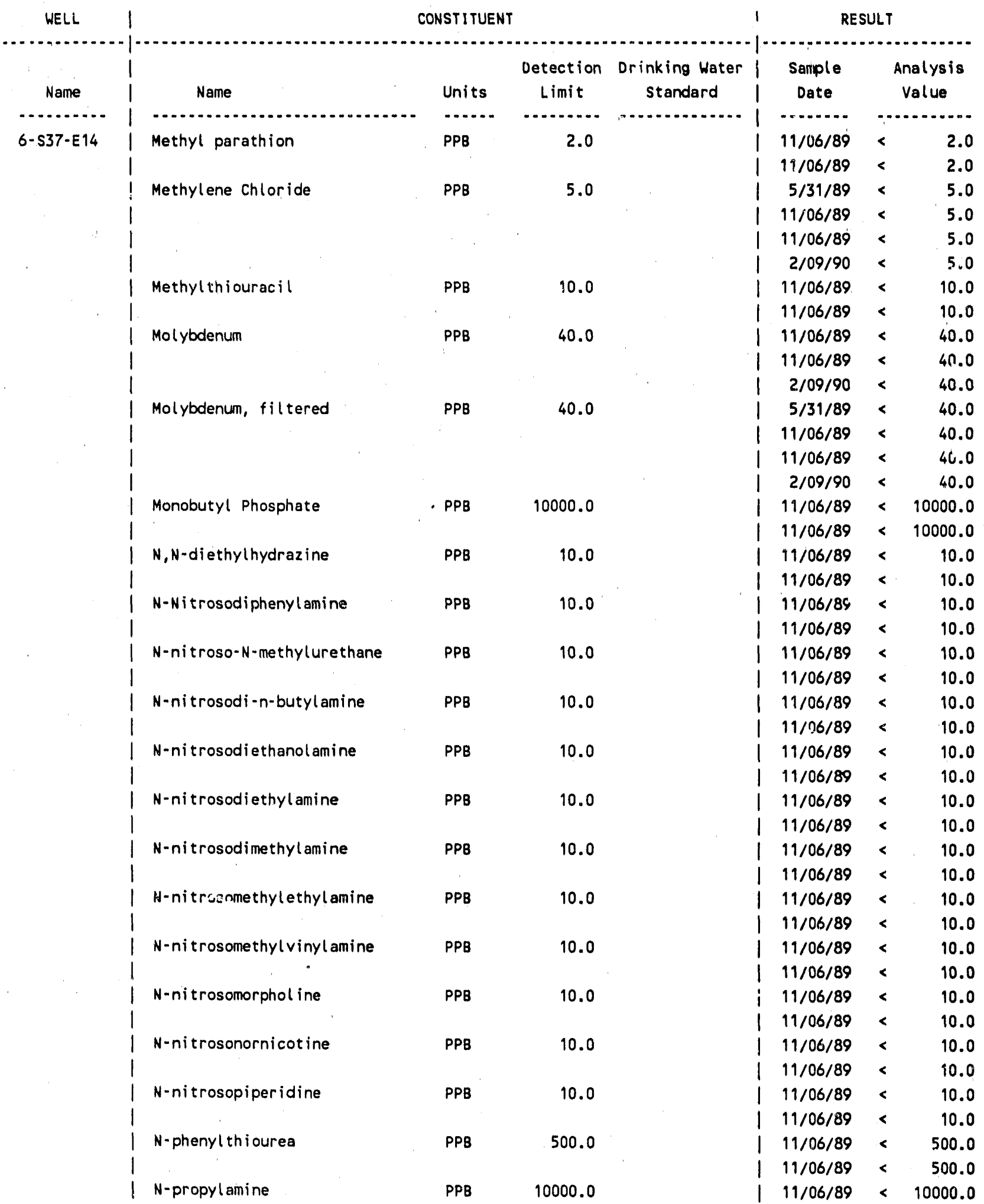


WHC-MR. 0229

Geosciences Group PARADOX Database

$11 / 26 / 90$

Well Result Report

Page 17

1100 Area Groundwater Monitoring

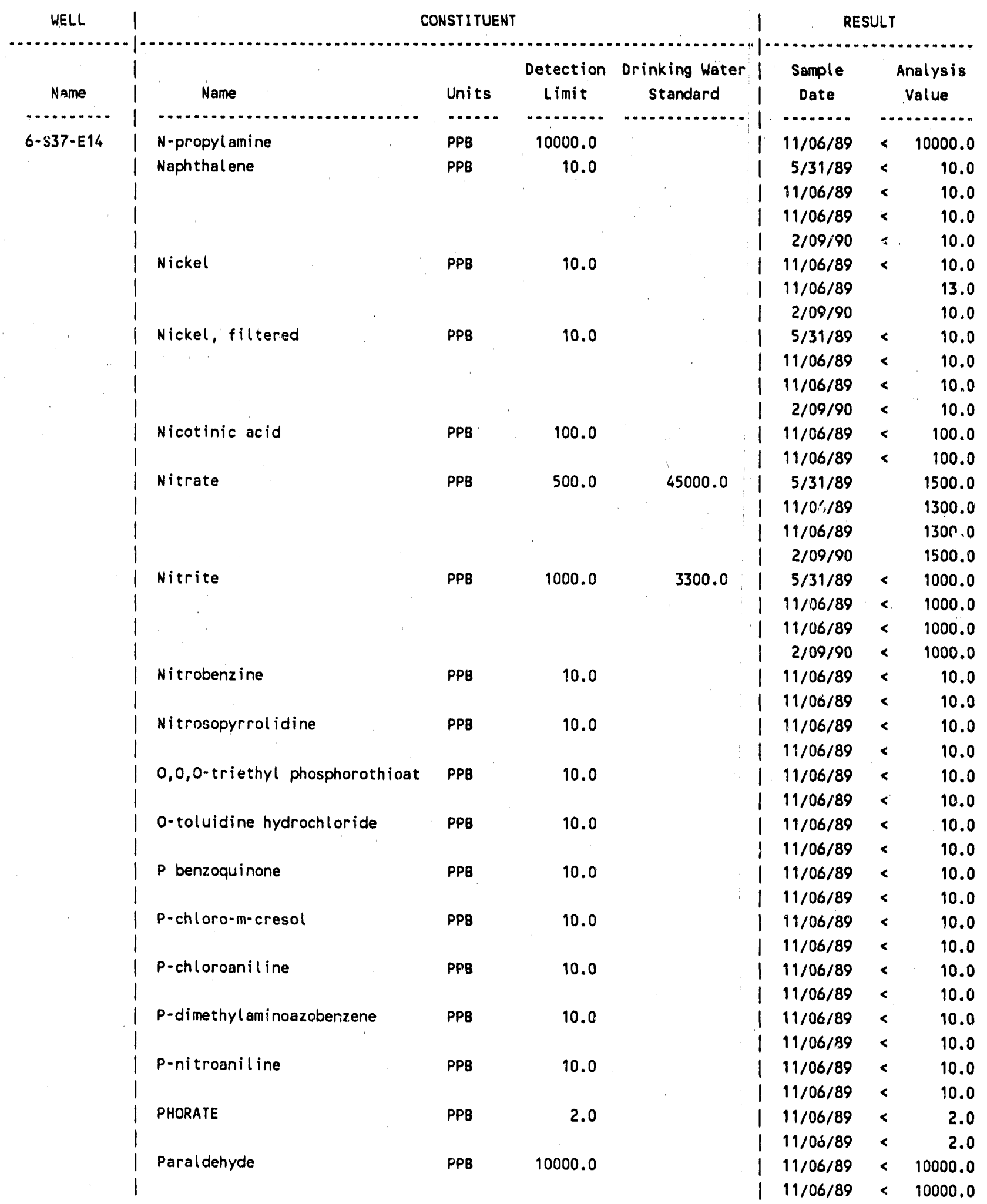


WHC-MR- 0229

Geosciences Group PARADOX Database

$11 / 26 / 90$

Well Result Report

Page 18

1100 Area Groundwater Monitoring

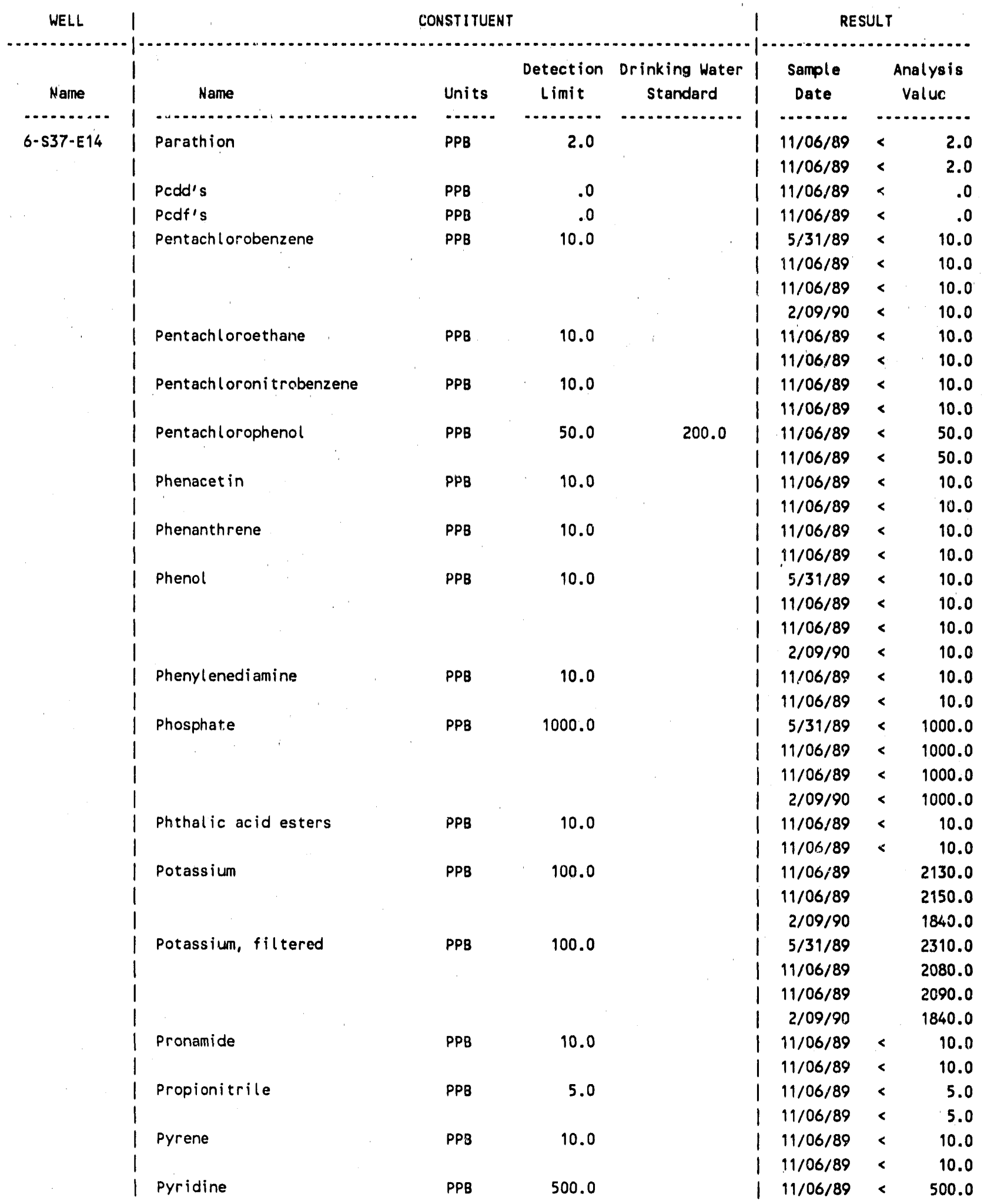




\section{WHC-MR. 0229}

$11 / 26 / 90$

G.yosciences Group PARADOX Database

Well Result Report

1100 Area Groundwater Monitoring

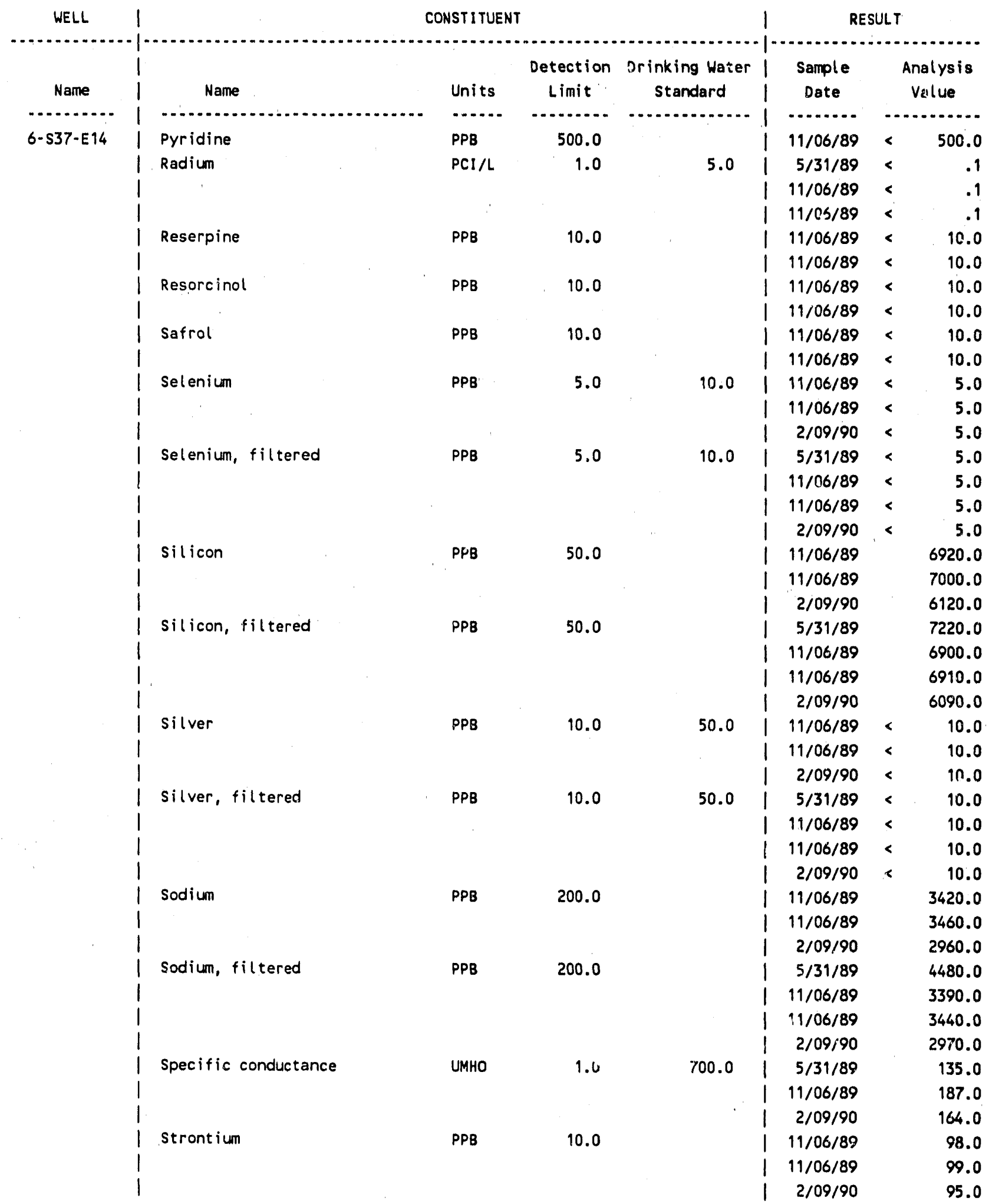


WHC-MR- 0229

$11 / 26 / 90$

Geosciences Group PARADOX Database

Hell Result Report

Page 20

1100 Area Groundwater Monitoring

\begin{tabular}{|c|c|c|c|c|c|c|c|c|c|}
\hline \multirow{2}{*}{$\begin{array}{c}\text { HELL } \\
\text { Name }\end{array}$} & \multicolumn{5}{|c|}{ CONSTI TUENT } & 1 & \multicolumn{3}{|c|}{ RESULT } \\
\hline & $\begin{array}{l}1 \\
1\end{array}$ & Name & Units & $\begin{array}{l}\text { Detection } \\
\text { Limit }\end{array}$ & $\begin{array}{l}\text { Orinking water } \\
\text { Standard }\end{array}$ & 1 & $\begin{array}{l}\text { Sample } \\
\text { Date }\end{array}$ & & $\begin{array}{l}\text { Analysis } \\
\text { Value }\end{array}$ \\
\hline ........... & 1 & 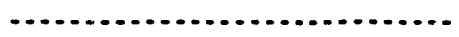 & ...... & .......... & $\ldots \ldots \ldots \ldots \ldots$ & 1 & .......... & & .......... \\
\hline \multirow[t]{44}{*}{ 6-537-E14 } & 1 & strontium, filtered & PPB & 10.0 & & i & $5 / 31 / 89$ & & 106.0 \\
\hline & 1 & & & & & i & $11 / 06 / 89$ & & 98.0 \\
\hline & 1 & & & & & 1 & $11 / 06 / 89$ & & 99.0 \\
\hline & 1 & & & & & 1 & $2 / 09 / 90$ & & 93.0 \\
\hline & 1 & Strychnine & PPB & 50.0 & & 1 & 11/06/oy & $<$ & 50.0 \\
\hline & 1 & & & & & 1 & $11 / 06 / 89$ & $<$ & 50.0 \\
\hline & $i$ & Styrene & PPB & 5.0 & 5.0 & I & $11 / 06 / 89$ & $<$ & 5.0 \\
\hline & 1 & & & & & 1 & $11 / 06 / 89$ & $<$ & 5.0 \\
\hline & 1 & Sulfate & PPB & 500.0 & 250000.0 & I & $5 / 31 / 89$ & & 14800.0 \\
\hline & 1 & & & & & I & $11 / 06 / 89$ & & 11100.0 \\
\hline & 1 & & & & & 1 & $11 / 06 / 89$ & & 11100.0 \\
\hline & 1 & & & & & 1 & $2 / 09 / 90$ & & 19500.0 \\
\hline & 1 & Sulfide & PPB & 1000.0 & & 1 & $11 / 06 / 89$ & $<$ & 1000.0 \\
\hline & 1 & & & & & 1 & $11 / 06 / 89$ & $<$ & 1000.0 \\
\hline & 1 & Sym-trinitrobenzene & PPB & 10.0 & & 1 & $11 / 06 / 89$ & $<$ & 10.0 \\
\hline & 1 & & & & & 1 & $11 / 06 / 89$ & $<$ & 10.0 \\
\hline & 1 & Tetrachloroethylene & PPB & 5.0 & 5.0 & I & $5 / 31 / 89$ & $<$ & 5.0 \\
\hline & 1 & & & & & 1 & $11 / 06 / 89$ & $<$ & 5.0 \\
\hline & 1 & & & & & 1 & $11 / 06 / 89$ & $<$ & 5.0 \\
\hline & 1 & & & & & 1 & $2 / 09 / 90$ & $<$ & 5.0 \\
\hline & 1 & Tetraethylpyrophosphate & PPB & 2.0 & & 1 & $11 / 06 / 89$ & $<$ & 2.0 \\
\hline & 1 & & & & & 1 & $11 / 06 / 89$ & $<$ & 2.0 \\
\hline & 1 & Tetrahydrofuran & PPB & 10.0 & & 1 & $5 / 31 / 89$ & $<$ & 10.0 \\
\hline & 1 & & & & & 1 & $11 / 06 / 89$ & $<$ & 10.0 \\
\hline & 1 & & & & & I & $11 / 06 / 89$ & $<$ & 10.0 \\
\hline & 1 & & & & & 1 & $2 / 09 / 90$ & $<$ & 10.0 \\
\hline & 1 & Thallium & PPB & 5.0 & & 1 & $11 / 06 / 89$ & $<$ & 5.0 \\
\hline & 1 & & & & & 1 & $11 / 06 / 89$ & $<$ & 5.0 \\
\hline & 1 & & & & & 1 & $2 / 09 / 90$ & $<$ & 5.0 \\
\hline & 1 & Thallium, filtered & PPB & 5.0 & & I & $5 / 31 / 89$ & $<$ & 5.0 \\
\hline & 1 & & & & & 1 & $11 / 06 / 89$ & $<$ & 5.0 \\
\hline & 1 & & & & & I & $11 / 06 / 89$ & $<$ & 5.0 \\
\hline & 1 & & & & & 1 & $2 / 09 / 90$ & $<$ & 5.0 \\
\hline & 1 & Thiofanox & PPB & 10.0 & & 1 & $11 / 06 / 89$ & $<$ & 10.0 \\
\hline & 1 & & & & & 1 & $11 / 06 / 89$ & $<$ & 10.0 \\
\hline & 1 & Thiourea & PPB & 200.0 & & 1 & $11 / 06 / 89$ & $<$ & 200.0 \\
\hline & $i$ & & & & & 1 & $11 / 06 / 89$ & $<$ & 200.0 \\
\hline & 1 & Thiuram & PPB & 10.0 & & 1 & $11 / 06 / 89$ & $<$ & 10.0 \\
\hline & 1 & & & & & 1 & $11 / 06 / 89$ & $<$ & 10.0 \\
\hline & 1 & Tin & PPB & 30.0 & & 1 & $11 / 06 / 89$ & $<$ & 30.0 \\
\hline & 1 & & & & & 1 & $11 / 06 / 89$ & $<$ & 30.0 \\
\hline & 1 & & & & & 1 & $2 / 09 / 90$ & $<$ & 30.0 \\
\hline & 1 & Tin, filtered & PPB & 30.0 & & 1 & $5 / 31 / 89$ & $<$ & 30.0 \\
\hline & 1 & & & & & I & $11 / 06 / 89$ & $<$ & 30.0 \\
\hline
\end{tabular}




\section{WHC.MR. 0229}

Well Result Report

1100 Area Groundwater Monitoring

\begin{tabular}{|c|c|c|c|c|c|c|c|c|c|}
\hline WELL & 1 & & NSTITUEN & & & 1 & & & \\
\hline Name & 1 & Name & Units & $\begin{array}{l}\text { Detection } \\
\text { Limit }\end{array}$ & $\begin{array}{c}\text { Drinking Water } \\
\text { standard }\end{array}$ & 1 & $\begin{array}{l}\text { Sample } \\
\text { Date }\end{array}$ & & $\begin{array}{l}\text { lalysis } \\
\text { lalue }\end{array}$ \\
\hline .......... & i & $\cdots \ldots \ldots \ldots \ldots \ldots \ldots \ldots$ & $\cdots \cdot . \cdot$ & …...... & (n........... & 1 & …..... & & (........ \\
\hline $6.537-E 14$ & 1 & Tin, filtered & PPB & 30.0 & & 1 & $11 / 06 / 89$ & $<$ & 30.0 \\
\hline & 1 & & & & & 1 & $2 / 09 / 90$ & $<$ & 30.0 \\
\hline & 1 & ritanium & PPB & 60.0 & & 1 & $11 / 06 / 89$ & $<$ & 60.0 \\
\hline & 1 & & & & & 1 & $11 / 06 / 89$ & $<$ & 60.0 \\
\hline & 1 & & & & & i & $2 / 09 / 90$ & « & 60.0 \\
\hline & 1 & Titanium, filtered & PPB & 60.0 & & 1 & $5 / 31 / 89$ & $<$ & 60.0 \\
\hline & $i$ & & & & & i & $11 / 06 / 89$ & $<$ & 60.0 \\
\hline & 1 & & & & & 1 & $11 / 06 / 89$ & $<$ & 60.0 \\
\hline & $!$ & & & & & 1 & $2 / 09 / 90$ & $<$ & 60.0 \\
\hline & 1 & Toluene & PPB & 5.0 & 2000.0 & I & $5 / 31 / 89$ & $<$ & 5.0 \\
\hline & 1 & & & & & 1 & $11 / 06 / 89$ & $<$ & 5.0 \\
\hline & 1 & & & & & 1 & $11 / 06 / 89$ & $<$ & 5.0 \\
\hline & 1 & & & & & I & $2 / 09 / 90$ & $<$ & 5.0 \\
\hline & 1 & Toluenediamine & PPB & 10.0 & & 1 & $11 / 06 / 89$ & $<$ & 10.0 \\
\hline & 1 & & & & & 1 & $11 / 06 / 89$ & $<$ & 10.0 \\
\hline & 1 & Total Organic Halogen, Low Det & PPB & 10.0 & & 1 & $5 / 31 / 89$ & & 34.0 \\
\hline & 1 & & & & & 1 & $11 / 06 / 89$ & & 22.0 \\
\hline & 1 & & & & & 1 & $2 / 09 / 90$ & $<$ & 6.0 \\
\hline & 1 & Total carbon & PPB & 1000.0 & & 1 & $5 / 31 / 89$ & & 20600.0 \\
\hline & i & & & & & i & $11 / 06 / 89$ & & 19400.0 \\
\hline & 1 & & & & & 1 & $11 / 06 / 89$ & & 19500.0 \\
\hline & 1 & & & & & 1 & $2 / 09 / 90$ & & 18100.0 \\
\hline & 1 & Total organic carbon & PPB & 1000.0 & & 1 & $5 / 31 / 89$ & $<$ & 800.0 \\
\hline & 1 & & & & & I & $11 / 06 / 89$ & $<$ & 800.0 \\
\hline & 1 & & & & & 1 & $2 / 09 / 90$ & $<$ & 700.0 \\
\hline & 1 & Toxaphene & PAB & 1.0 & 5.0 & 1 & $5 / 31 / 89$ & $<$ & 1.0 \\
\hline & i & & & & & i & $11 / 06 / 89$ & $<$ & 1.0 \\
\hline & 1 & & & & & 1 & $11 / 06 / 89$ & $<$ & 1.0 \\
\hline & 1 & & & & & 1 & $2 / 09 / 90$ & $<$ & 1.0 \\
\hline & 1 & Trans-1,2-dichloroe thene & PPB & 5.0 & 70.0 & 1 & $5 / 31 / 89$ & $<$ & 5.0 \\
\hline & 1 & & & & & 1 & $11 / 06 / 89$ & $<$ & 5.0 \\
\hline & 1 & & & & & 1 & $11 / 06 / 89$ & $<$ & 5.0 \\
\hline & 1 & & & & & 1 & $2 / 09 / 90$ & $<$ & 5.0 \\
\hline & 1 & Tributylphosphoric Acid & PPB & 10.0 & & 1 & $5 / 3 ? / 89$ & $<$ & 10.0 \\
\hline & 1 & & & & & 1 & $11 / 06 / 89$ & \& & 10.0 \\
\hline & 1 & & & & & 1 & $11 / 06 / 89$ & $<$ & 10.0 \\
\hline & 1 & & & & & 1 & $2 / 09 / 90$ & $<$ & 10.0 \\
\hline & 1 & Trichloroethylene & PPB & 5.0 & 5.0 & 1 & $5 / 31 / 89$ & $<$ & 5.0 \\
\hline & 1 & & & & & 1 & $11 / 06 / 89$ & $<$ & 5.0 \\
\hline & 1 & & & & & 1 & $11 / 06 / 89$ & $<$ & 5.0 \\
\hline & 1 & & & & & 1 & $2 / 09 / 90$ & $<$ & 5.0 \\
\hline & 1 & Trichloromethanethiol & PPB & 10.0 & & 1 & $11 / 06 / 89$ & $<$ & 10.0 \\
\hline & I & & & & & i & $11 / 06 / 89$ & $<$ & 10.0 \\
\hline & 1 & Trichloromonof luorome thane & PPB & 10.0 & & 1 & $11 / 06 / 89$ & $<$ & 10.0 \\
\hline
\end{tabular}




\section{WHC-MR- 0229}

$11 / 26 / 90$

Geosciences Group PARADOX Database

Well Result Report

1100 Area Groundwater Monitoring

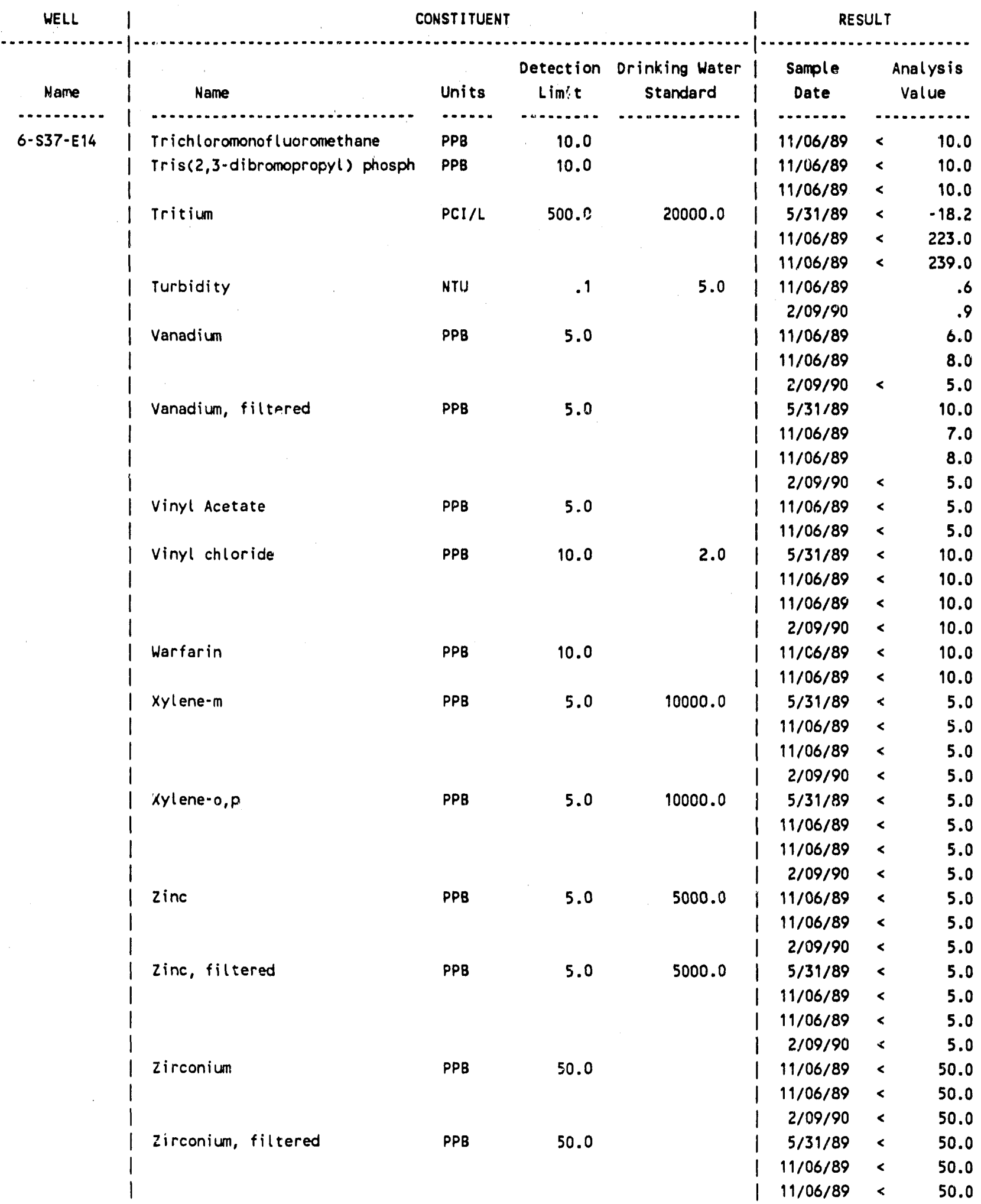




\section{WHC-MR- 0229}

$11 / 26 / 90$

Geosciences Group PARADOX Database

Hell Result Report

1100 Area Groundwater Monitoring

\begin{tabular}{|c|c|c|c|c|c|c|c|c|}
\hline \multirow{2}{*}{$\begin{array}{l}\text { WELL } \\
\text { Name }\end{array}$} & \multicolumn{5}{|c|}{ CONSTI TUENT } & \multicolumn{3}{|c|}{ RESULT } \\
\hline & 1 & Name & Units & $\begin{array}{c}\text { Detectio } \\
\text { Limit }\end{array}$ & $\begin{array}{l}\text { Drinking Water } \\
\text { standard }\end{array}$ & $\begin{array}{l}\text { Sample } \\
\text { Date }\end{array}$ & & $\begin{array}{l}\text { lysis } \\
\text { lue }\end{array}$ \\
\hline & I & 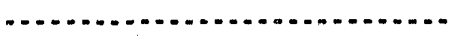 & ....... & -......... & 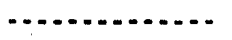 & -........ & & \\
\hline \multirow[t]{26}{*}{ 6-537-E14 } & 1 & Zirconium, filtered & PPB & 50.0 & & $2 / 09 / 90$ & $<$ & 50.0 \\
\hline & $i$ & dibromochlorome thane & PPB & 5.0 & 100.0 & $11 / 06 / 89$ & $<$ & 5.0 \\
\hline & 1 & & & & & | $11 / 06 / 89$ & $<$ & 5.0 \\
\hline & 1 & $\mathrm{~m}-\mathrm{Ni}$ troaniline & PPB & 10.0 & & I 11/06/89 & $<$ & 10.0 \\
\hline & 1 & & & & & | 11/06/89 & $<$ & 10.0 \\
\hline & 1 & o-Mitroaniline & PPB & 10.0 & & | $11 / 06 / 89$ & $<$ & 10.0 \\
\hline & 1 & & & & & | 11/06/89 & $<$ & 10.0 \\
\hline & 1 & O-Nitrophenol & PPB & 10.0 & & | 11/06/89 & $<$ & 10.0 \\
\hline & 1 & & & & & $111 / 06 / 89$ & $<$ & 10.0 \\
\hline & 1 & p-oichlorobenzene & PPB & 5.0 & 750.0 & I $5 / 31 / 89$ & $<$ & 5.0 \\
\hline & 1 & & & & & $1 \quad 11 / 06 / 89$ & $<$ & 5.0 \\
\hline & 1 & & & & & I 11/06/89 & $<$ & 5.0 \\
\hline & 1 & & & & & $1 \quad 2 / 09 / 90$ & $<$ & 5.0 \\
\hline & 1 & & & 10.0 & 750.0 & $5 / 31 / 89$ & $<$ & 10.0 \\
\hline & 1 & & & & & I $11 / 06 / 89$ & $<$ & 10.0 \\
\hline & 1 & & & & & | 11/06/89 & $<$ & 10.0 \\
\hline & 1 & & & & & $1 \quad 2 / 09 / 90$ & $<$ & 10.0 \\
\hline & 1 & p-Nitrophenol & PPB & 10.0 & & | $i 1 / 06 / 89$ & $<$ & 10.0 \\
\hline & 1 & & & & & $111 / 06 / 89$ & $<$ & 10.0 \\
\hline & 1 & pH, field Measurement & & .1 & 8.5 & $5 / 31 / 89$ & & 7.5 \\
\hline & 1 & & & & & | $11 / 06 / 89$ & & 8.0 \\
\hline & 1 & & & & & $2 / 09 / 90$ & & 8.1 \\
\hline & I & pH, Laboratory Measurement & & .0 & 8.5 & $5 / 31 / 89$ & & 8.1 \\
\hline & 1 & & & & & | 11/06/89 & & 7.9 \\
\hline & 1 & & & & & $1 \quad 2 / 09 / 90$ & & 7.8 \\
\hline & 1 & & & & & 1 & & \\
\hline \multirow[t]{18}{*}{ 6-540-E14 } & 1 & 0,0-Diethyl-0,2-pyrazinyl phos & PPB & 10.0 & & $11 / 03 / 89$ & $<$ & 10.0 \\
\hline & 1 & $1,1,1,2$ - tetrachlorethane & PPB & 10.0 & & $11 / 03 / 89$ & $<$ & 10.0 \\
\hline & 1 & $1,1,1-\operatorname{trichloroethane}$ & PPB & 5.0 & 200.0 & $5 / 30 / 89$ & $<$ & 5.0 \\
\hline & I & & & & & | 11/03/89 & $<$ & 5.0 \\
\hline & 1 & & & & & $2 / 09 / 90$ & $<$ & 5.0 \\
\hline & 1 & 1,1,2.2-tetrachlorethane & PPB & 5.0 & & | 11/03/89 & $<$ & 5.0 \\
\hline & 1 & 1,1,2-trichloroethane & PPB & 5.0 & & $5 / 30 / 89$ & $<$ & 5.0 \\
\hline & 1 & & & & & I 11/03/89 & $<$ & 5.0 \\
\hline & 1 & & & & & 1 $2 / 09 / 90$ & $<$ & 5.0 \\
\hline & 1 & 1,1-dichloroethane & PPB & 5.0 & & $5 / 30 / 89$ & $<$ & 5.0 \\
\hline & 1 & & & & & | 11/03/89 & $<$ & 5.0 \\
\hline & 1 & & & & & 1 $2 / 09 / 90$ & $<$ & 5.0 \\
\hline & 1 & 1,1-dichloroethylene & PPB & 10.0 & 7.0 & I 11/03/89 & $<$ & 10.0 \\
\hline & 1 & 1,1-dimethylhydrazine & PPB & 10.0 & & | 11/03/89 & $<$ & 10.0 \\
\hline & 1 & $1,2,3,4$-tetrachlorobenzene & PPB & 10.0 & & I $5 / 30 / 89$ & $<$ & 10.0 \\
\hline & 1 & & & & & | 11/03/89 & $<$ & 10.0 \\
\hline & 1 & & & & & $12 / 09 / 90$ & $<$ & 10.0 \\
\hline & 1 & 1,2,3,5-tetrachlorobenzene & PPB & 10.0 & & $5 / 30 / 89$ & $<$ & 10.0 \\
\hline
\end{tabular}




\section{WHC-MR. 0229}

Well Result Report

1100 Area Groundwater Monitoring

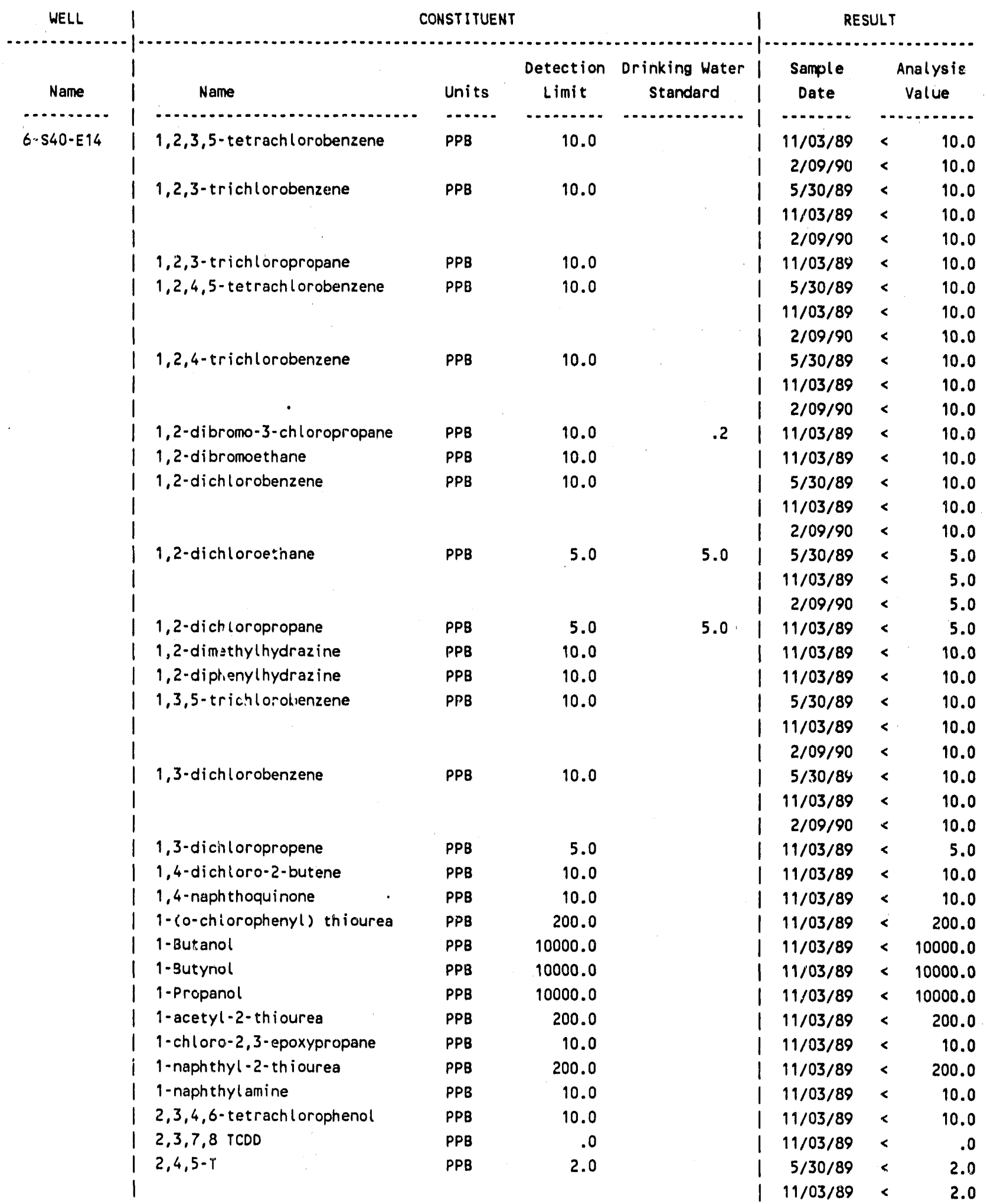




\section{WHC-MR. 0229}

$11 / 26 / 90$

Geosciences Group PARADOX Database

Well Result Report

Page 25

1100 Area Groundwater Monitoring

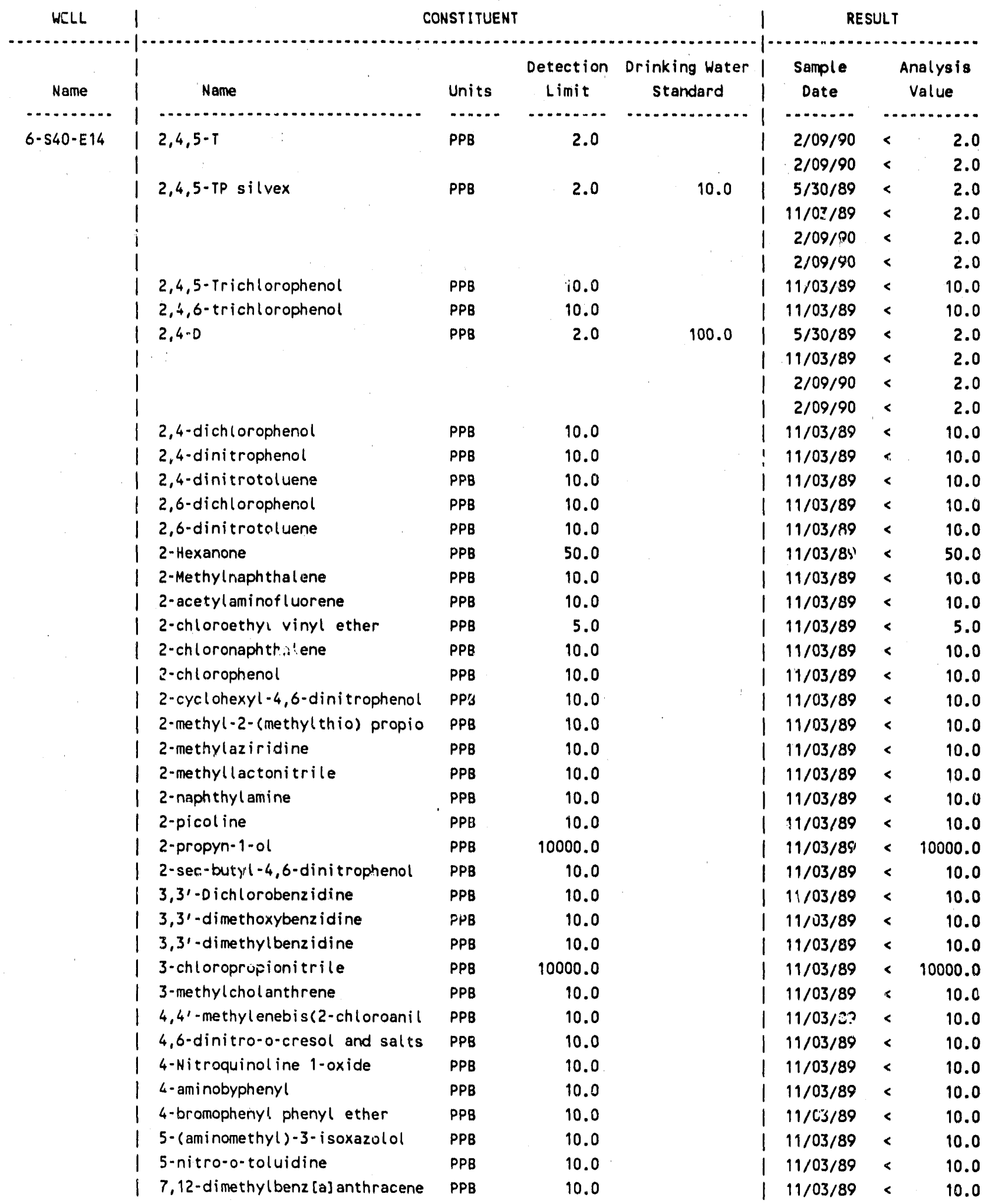


WHC-MR. 0229

$11 / 26 / 90$

Geosciences Group PARADOX Database

Page 2/J

Well Result Report

1100 Area Groundwater Monitoring

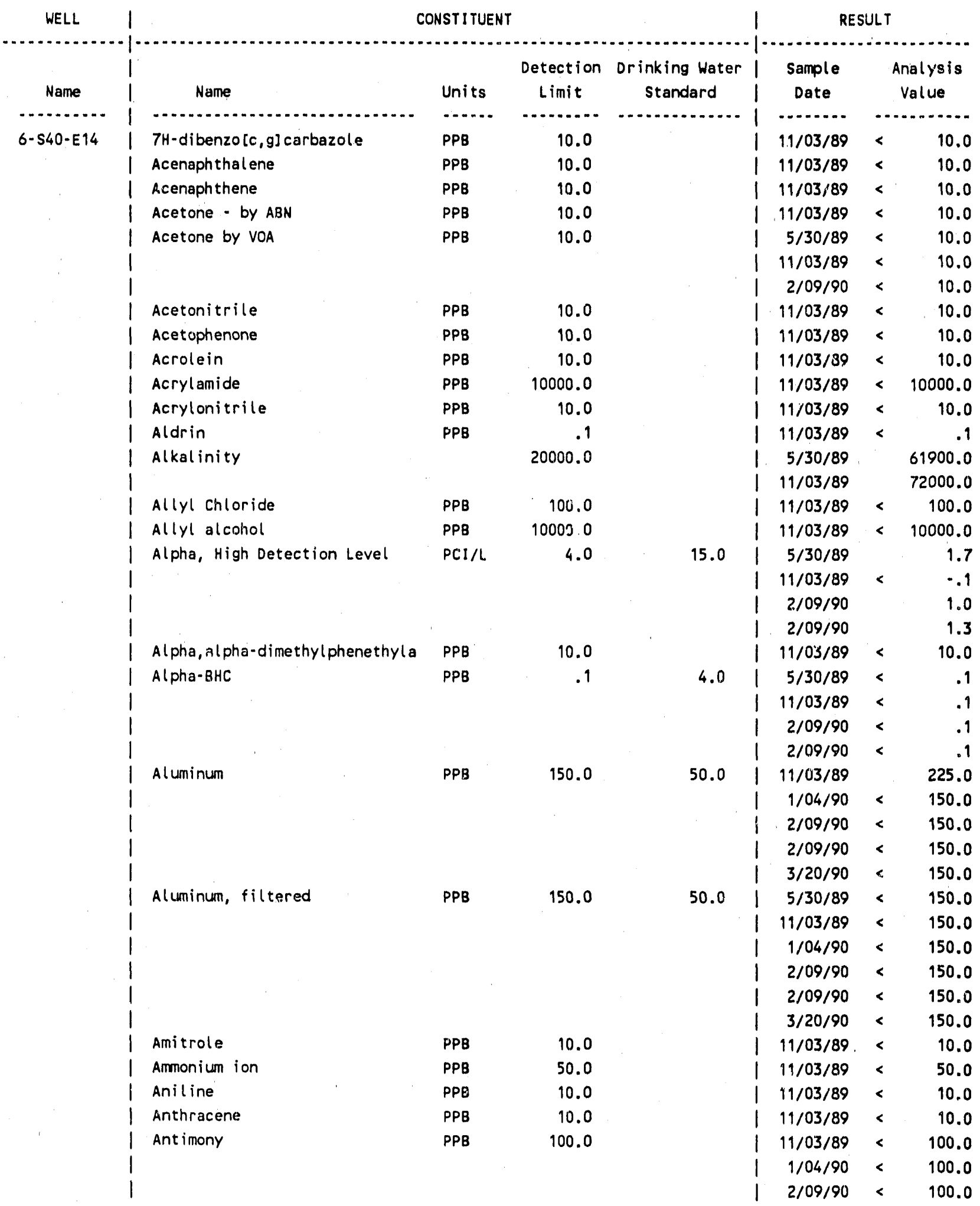


WHC.MR. 0229

$11 / 26 / 90$

Geosciences Group PARADOX Database

Page 27

Well Result Report

1100 Area Groundwater Monitoring

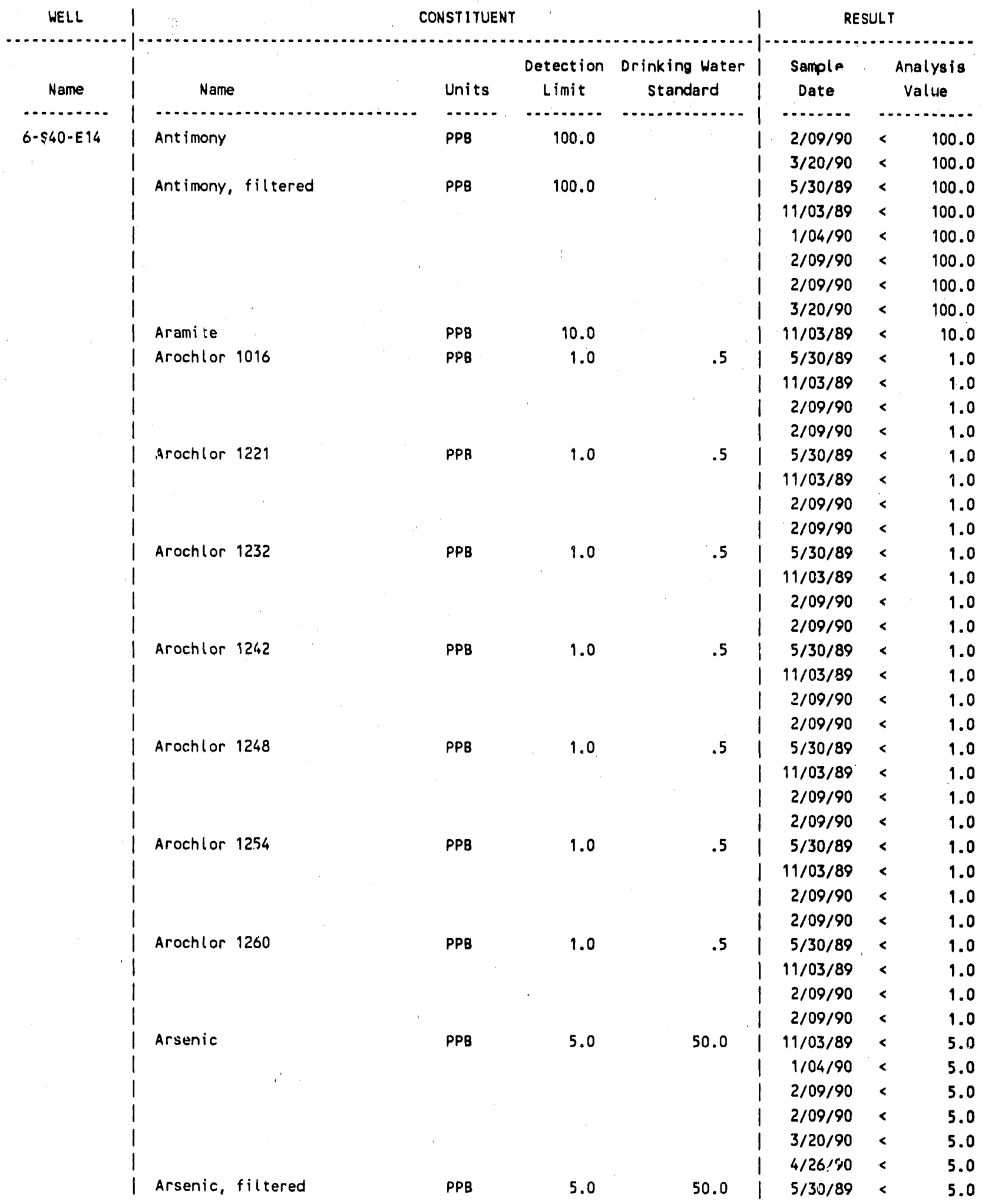


WHC-MR- 0229

$11 / 26 / 90$

Geosciences Group PARADOX Database

Page 28

Well Result Report

1100 Area Groundwater Monitoring

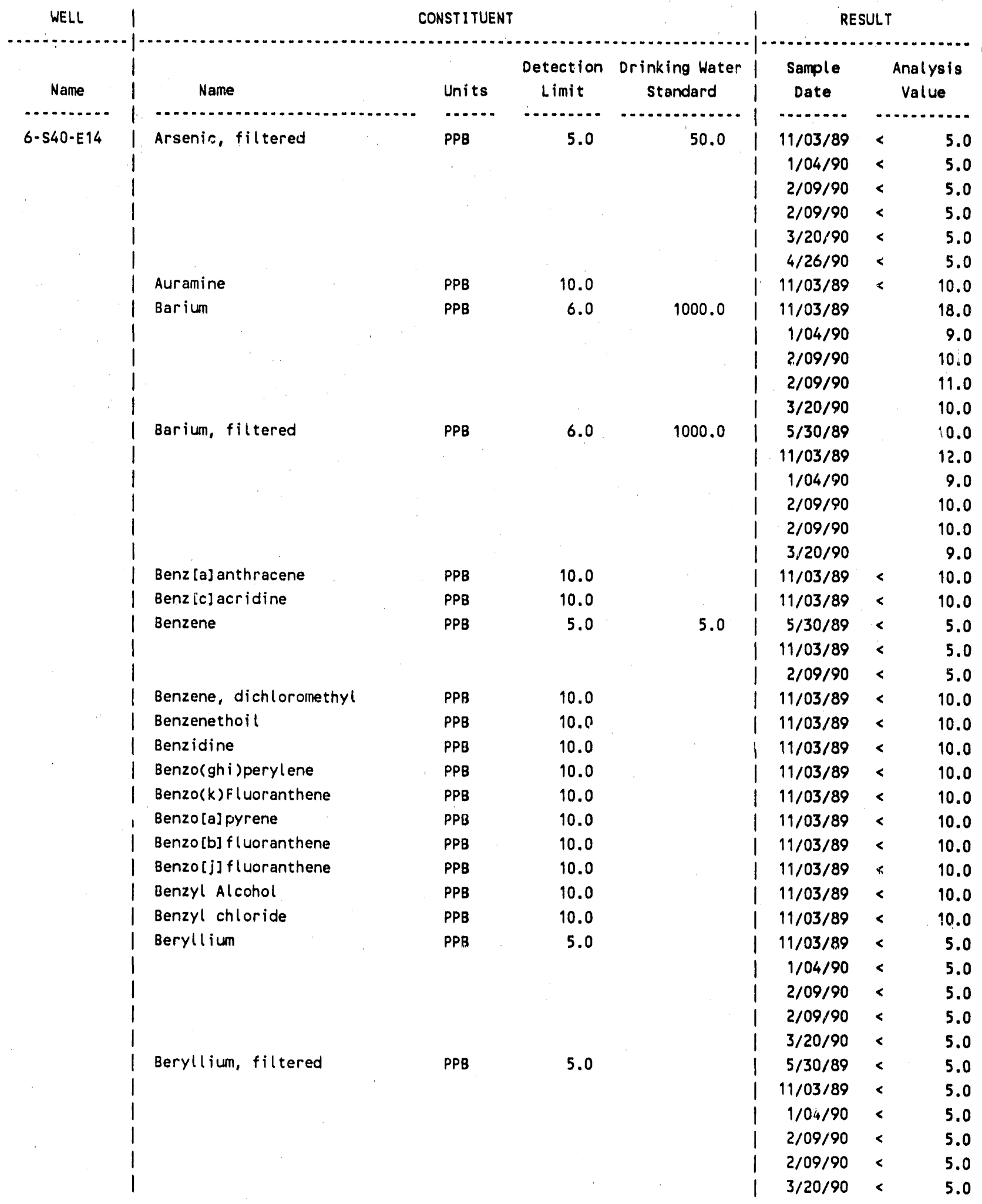


WHC-MR. 0229

Geosciences Group PARADOX Database

$11 / 26 / 90$

Well Result Report

Page 29

1100 Area Graundwater Monitoring

\begin{tabular}{|c|c|c|c|c|c|c|c|}
\hline WELL & \multicolumn{4}{|c|}{ CONSTITUENT } & \multicolumn{3}{|c|}{ RESULT } \\
\hline Name & 1 & Units & $\begin{array}{l}\text { Detection } \\
\text { Limit }\end{array}$ & $\begin{array}{c}\text { Drinking Water } \\
\text { standard }\end{array}$ & $\begin{array}{l}\text { Sample } \\
\text { Date }\end{array}$ & & $\begin{array}{l}\text { nalysis } \\
\text { Value }\end{array}$ \\
\hline …...... & | & ...... & .......... & (n................ & ......... & & .......... \\
\hline $6-540-E 14$ & I Beta-BHC & PPB & .1 & 4.0 & $5 / 30 / 89$ & $<$ & .1 \\
\hline & 1 & & & & | 11/03/89 & $<$ & .1 \\
\hline & 1 & & & & | $2 / 09 / 90$ & $<$ & .1 \\
\hline & 1 & & & & $2 / 09 / 90$ & $<$ & .1 \\
\hline & | Bis(2-chloro-1-methylethyl)eth & PPB & 10.0 & & | 11/03/89 & $<$ & 10.0 \\
\hline & isis(2-chloroethoxy) methane & PPB & 10.0 & & | 11/03/89 & $<$ & 10.0 \\
\hline & I sis(2-chloroethyl) ether & PPB & 10.0 & & $11 / 03 / 89$ & $<$ & 10.0 \\
\hline & I Bis(2-chloroisopropyl)ether & PPB & 10.0 & & | 11/03/89 & $<$ & 10.0 \\
\hline & I Bis(2-ethylhexyl) phthalate & PPB & 10.0 & & | 11/03/89 & $<$ & 10.0 \\
\hline & | Bis(chloromethyl) ether & PPB & 5.0 & & | 11/03/89 & $<$ & 5.0 \\
\hline & I Boron & PPB & 10.0 & & | 11/03/89 & & 12.0 \\
\hline & 1 & & & & $1 / 04 / 90$ & $<$ & 10.0 \\
\hline & 1 & & & & $2 / 09 / 70$ & & 24.0 \\
\hline & 1 & & & & $2 / 09 / 90$ & & 37.0 \\
\hline & i & & & & $3 / 20 / 90$ & & 22.0 \\
\hline & I Boron, filtered & PPB & 10.0 & & $5 / 30 / 89$ & $<$ & 10.0 \\
\hline & 1 & & & & | 11/03/89 & & 12.0 \\
\hline & 1 & & & & | $1 / 04 / 90$ & $<$ & 10.0 \\
\hline & 1 & & & & $2 / 09 / 90$ & & 17.0 \\
\hline & 1 & & & & $2 / 09 / 90$ & & 34.0 \\
\hline & 1 & & & & $3 / 20 / 90$ & & 18.0 \\
\hline & I Bromide & PPB & 1000.0 & & $5 / 30 / 89$ & $<$ & 1000.0 \\
\hline & 1 & & & & | 11/03/89 & $<$ & 1000.0 \\
\hline & 1 & & & & $1 / 04 / 90$ & $<$ & 1000.0 \\
\hline & 1 & & & & $2 / 09 / 90$ & $<$ & 1000.0 \\
\hline & 1 & & & & $2 / 09 / 90$ & $<$ & 1000.0 \\
\hline & 1 & & & & $3 / 20 / 90$ & $<$ & 1000.0 \\
\hline & 1 & & & & $14 / 26 / 90$ & $<$ & 1000.0 \\
\hline & I Bromoacetone & PPB & 5.0 & & | 11/03/89 & $<$ & $5 . n$ \\
\hline & I Bromodichlorome thane & PPB & 5.0 & 100.0 & | 11/03/89 & $<$ & 5.0 \\
\hline & I Bromoform & PPB & 5.0 & 100.0 & | 11/03/89 & $<$ & 5.0 \\
\hline & I Butyl benzyl phthalate & PPB & 10.0 & & 1. 11/03/89 & $<$ & 10.0 \\
\hline & I Cadmium & PPB & 2.0 & 10.0 & I 11/03/89 & $<$ & 2.0 \\
\hline & 1 & & & & $1 / 04 / 90$ & $<$ & 2.0 \\
\hline & 1 & & - & & $2 / 09 / 90$ & $<$ & 2.0 \\
\hline & 1 & & & & $2 / 09 / 90$ & $<$ & 2.0 \\
\hline & 1 & & & & $3 / 20 / 90$ & $<$ & 2.0 \\
\hline & | Cadmium, filtered & PPB & 2.0 & 10.0 & $5 / 30 / 89$ & $<$ & 2.0 \\
\hline & 1 & & & & | 11/03/89 & $<$ & 2.0 \\
\hline & 1 & & & & $1 \quad 1 / 04 / 90$ & $<$ & 2.0 \\
\hline & 1 & & & & $2 / 09 / 90$ & $<$ & 2.0 \\
\hline & 1 & & & & $2 / 09 / 90$ & $<$ & 2.0 \\
\hline & 1 & & & & $3 / 20 / 90$ & $<$ & 2.0 \\
\hline & I Calcium & PPB & 50.0 & & | 11/03/89 & & 23900.0 \\
\hline
\end{tabular}


WHC-MR- 0229

Geosciences Group PARADOX Database

$11 / 26 / 90$

Well Result Report

Page 30

1100 Area Groundwater Monitoring

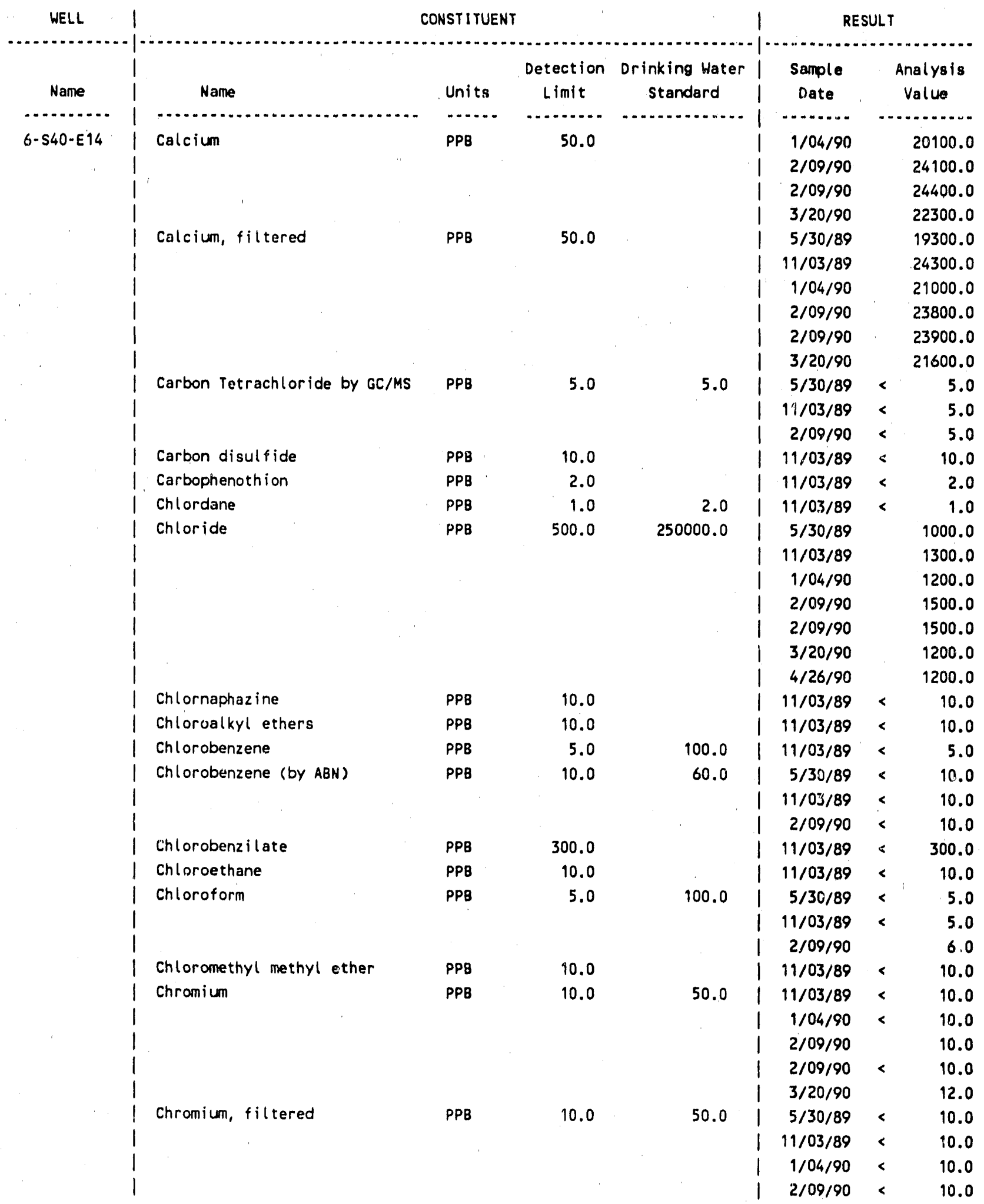


WHC-MR- 0229

Geosciences Group PARADOX Database.

$11 / 26 / 90$

Well Result Report

Page 31

1100 Area Groundwater Monitoring

\begin{tabular}{|c|c|c|c|c|c|c|c|c|}
\hline WELL & 1 & \multicolumn{4}{|c|}{ CONST ITUENT } & \multicolumn{3}{|c|}{ RESULT } \\
\hline & i & & & Detection & Drinking Watur & Sample & & lalysis \\
\hline Name & i & Name & Units & Limit & standard & Date & & lalue \\
\hline - n.......... & 1 & (n) & $\cdots \ldots$ & $\cdots \ldots$ & (n) & - n........... & & (n)............ \\
\hline 6-S40-E14 & i & Chromium, filtered & PPB & 10.0 & 50.0 & $2 / 09 / 90$ & $<$ & 10.0 \\
\hline & 1 & & & & & $3 / 20 / 90$ & $<$ & 10.0 \\
\hline & i & Chrysene & PPB & 10.0 & & $11 / 03 / 89$ & $<$ & 10.0 \\
\hline & i & Citrus red & PPB & 1000.0 & & $11 / 03 / 89$ & $<$ & 1000.0 \\
\hline & i & Cobalt & PPB & 20.0 & & $11 / 03 / 89$ & $<$ & 20.0 \\
\hline & 1 & & & & & $1 / 04 / 90$ & $<$ & 20.0 \\
\hline & 1 & & & & & $2 / 09 / 90$ & $<$ & 20.0 \\
\hline & 1 & & & & & $2 / 09 / 90$ & $<$ & 20.0 \\
\hline & i & & & & & $3 / 20 / 90$ & $<$ & 20.0 \\
\hline & 1 & Cobalt, filtered & PPB & 20.0 & . & $5 / 30 / 89$ & $<$ & 20.0 \\
\hline & 1 & & & & & | 11/03/89 & $<$ & 20.0 \\
\hline & 1 & & & & & i $1 / 04 / 90$ & $<$ & 20.0 \\
\hline & 1 & . & & & & $2 / 09 / 90$ & $<$ & 20.0 \\
\hline & i & & & & & $2 / 09 / 90$ & $<$ & 20.0 \\
\hline & 1 & & & & & $3 / 20 / 90$ & $<$ & 20.0 \\
\hline & 1 & Coliform bacteria & MPN & 2.2 & 1.0 & | $11 / 03 / 89$ & $<$ & 2.2 \\
\hline & 1 & Copper & PPB & 10.0 & 1000.0 & $11 / 03 / 89$ & $<$ & 10.0 \\
\hline & 1 & & & & & $1 / 04 / 90$ & $<$ & 10.0 \\
\hline & 1 & & & & & $2 / 09 / 90$ & $<$ & 10.0 \\
\hline & 1 & & & & & $2 / 09 / 90$ & $<$ & 10.0 \\
\hline & 1 & & & & & $3 / 20 / 90$ & $<$ & 10.0 \\
\hline & 1 & Copper, filtered & PPB. & 10.0 & 1000.0 & $5 / 30 / 89$ & $<$ & 10.0 \\
\hline & 1 & & & & & | 11/03/89 & $<$ & 10.0 \\
\hline & 1 & & & & & $11 / 04 / 90$ & $<$ & 10.0 \\
\hline & i & & & & & $2 / 09 / 90$ & $<$ & 10.0 \\
\hline & i & & & & & $2 / 09 / 90$ & $<$ & 10.0 \\
\hline & 1 & & & & & $3 / 20 / 90$ & $<$ & 10.0 \\
\hline & 1 & Cresols & PPB & 10.0 & & $11 / 03 / 89$ & $<$ & 10.0 \\
\hline & 1 & Crotonaldehyde & PPB & 10.0 & & $11 / 03 / 89$ & $<$ & 10.0 \\
\hline & 1 & Cyanide & PPB & 10.0 & & $11 / 03 / 89$ & $<$ & 10.0 \\
\hline & 1 & DDD & PPB & .1 & & $11 / 03 / 89$ & $<$ & .1 \\
\hline & i & DDE & PPB & .1 & & $11 / 03 / 89$ & $<$ & .1 \\
\hline & 1 & DDT & PPB & .1 & & | 11/03/89 & $<$ & .1 \\
\hline & $i$ & Delta-BHC & PPB & .1 & 4.0 & i $5 / 30 / 89$ & $<$ & .1 \\
\hline & i & & & & & $11 / 03 / 89$ & $<$ & .1 \\
\hline & 1 & & & & & $12 / 09 / 90$ & $<$ & .1 \\
\hline & 1 & & & & & $2 / 09 / 90$ & $<$ & .1 \\
\hline & 1 & Di-n-butyl phthalate & PPB & 10.0 & & $11 / 03 / 89$ & $<$ & 10.0 \\
\hline & 1 & Di-n-octyl phthalate & PPB & 10.0 & & $11 / 03 / 89$ & $<$ & 10.0 \\
\hline & 1 & Di-n-propylnitrosamine & PPB & 10.0 & & $11 / 03 / 89$ & $<$ & 10.0 \\
\hline & i & Diallate & PPB & 10.0 & & $11 / 03 / 89$ & $<$ & 10.0 \\
\hline & I & Dibenz $[a, h]$ acridine & PPB & 10.0 & & $11 / 03 / 89$ & $<$ & 10.0 \\
\hline & $i$ & Dibenz $[a, h]$ anthracene & PPB & 10.0 & & $11 / 03 / 89$ & $<$ & 10.0 \\
\hline & 1 & Dibenz $[a, j]$ acridine & PPB & 10.0 & & | $11 / 03 / 89$ & $<$ & 10.0 \\
\hline
\end{tabular}




\section{WHC-MPP. 0229}

Well Result Report

1100 Area Groundwater Monitoring

\begin{tabular}{|c|c|c|c|c|c|c|c|}
\hline \multirow{2}{*}{$\begin{array}{c}\text { WELL } \\
\text { Name }\end{array}$} & \multicolumn{4}{|c|}{ CONSTITUENT } & \multicolumn{3}{|c|}{ RESULT } \\
\hline & Name & Units & $\begin{array}{l}\text { Detection } \\
\text { Limit }\end{array}$ & $\begin{array}{c}\text { Drinking Water } \\
\text { otandard }\end{array}$ & $\begin{array}{l}\text { Sample } \\
\text { Date }\end{array}$ & & $\begin{array}{l}\text { inalysis } \\
\text { Value }\end{array}$ \\
\hline n............. & | & $\cdots \cdots$ & n......... & (n) & | $\cdots \cdots . .$. & $\cdots$ & \\
\hline \multirow[t]{44}{*}{$6-540-E 14$} & | Dibenzo[a, e] pyrene & PPB & 10.0 & & | 11/03/89 & $<$ & 10.0 \\
\hline & Dibenzo [a, h] pyrene & PPB & 10.0 & & | 11/03/89 & $<$ & 10.0 \\
\hline & I Dibenzo[a,i]pyrene & PPB & 10.0 & & $11 / 03 / 89$ & $<$ & 10.0 \\
\hline & I Dibenzofuran & PPB & 10.0 & & | 11/03/89 & $<$ & 10.0 \\
\hline & I Dibromome thane & PPB & 10.0 & & | 11/03/89 & $<$ & 10.0 \\
\hline & I Dibutyl Phosphate & PPB & 10000.0 & & | 11/03/89 & $<$ & 10000.0 \\
\hline & | Dichlorodifluoromethane & PPB & 10.0 & & | 11/03/89 & $<$ & 10.0 \\
\hline & I Dieldrin & PPB & .1 & & | 11/03/89 & $<$ & .1 \\
\hline & I Diethyl phthalate & PPB & 10.0 & & | 11/03/89 & $<$ & 10.0 \\
\hline & I Diethylstilbesterol & PPB & 200.0 & & | 11/03/89 & $<$ & 200.0 \\
\hline & I Dihydrosafrole & PPB & 10.0 & & | 11/03/89 & $<$ & 10.0 \\
\hline & I Dimethoate & PPB & 2.0 & & 1 11/03/89 & $<$ & 2.0 \\
\hline & I Dimethyl phthalate & PPB & 10.0 & & | 11/03/89 & $<$ & 10.0 \\
\hline & I Dinitrobenzene & PPB & 10.0 & & | 11/03/89 & $<$ & 10.0 \\
\hline & I Dinoseb & PPB & 10.0 & & | 11/03/89 & $<$ & 10.0 \\
\hline & I Dioxane & PPB & 500.0 & & | 11/03/89 & $<$ & 500.0 \\
\hline & I Diphenytamine & PPB & 10.0 & & | 11/03/89 & $<$ & 10.0 \\
\hline & | Disulfoton & PPB & 2.0 & & | 11/03/89 & $<$ & 2.0 \\
\hline & I Endosulfan I & PPB & .1 & & | 11/03/89 & $<$ & .1 \\
\hline & I Endosulfan II & PPB & .1 & & | 11/03/89 & $<$ & .1 \\
\hline & | Endosulfan sulfate & PPB & .5 & & | 11/03/89 & $<$ & .5 \\
\hline & Endrin & PPB & .1 & .2 & $5 / 30 / 89$ & $<$ & .1 \\
\hline & 1 & & & & | 11/03/89 & $<$ & .1 \\
\hline & 1 & & & & | 2/09/90 & $<$ & .1 \\
\hline & 1 & & & & $2 / 09 / 90$ & $<$ & \\
\hline & | Ethanol & PPB & 10000.0 & & | 11/03/89 & $<$ & 10060.0 \\
\hline & I Ethyl benzene & PPB & 5.0 & 700.0 & | 11/03/89 & $<$ & 5.0 \\
\hline & | Ethyl carbamate & PPB & 10000.0 & & $11 / 03 / 89$ & $<$ & 10000.0 \\
\hline & | Ethyl cyanide & PPB & 10000.0 & & $11 / 03 / 89$ & $<$ & 10000.0 \\
\hline & I Ethyl methacrylate & PPB & 10.0 & & $11 / 03 / 89$ & $<$ & 10.0 \\
\hline & I Ethyl methanesul fonate & PPB & 10.0 & & | 11/03/89 & $<$ & 10.0 \\
\hline & I Ethylene Glycol & PPB & 10000.0 & & I 11/03/89 & $<$ & 10000.0 \\
\hline & I Ethylene glycol & PPB & 10000.0 & & | $5 / 30 / 89$ & $<$ & 10000.0 \\
\hline & 1 & & & & $1 / 04 / 90$ & $<$ & 10000.0 \\
\hline & 1 & & & & $2 / 09 / 90$ & $<$ & 10000.0 \\
\hline & 1 & & & & $3 / 20 / 90$ & $<$ & 10000.0 \\
\hline & 1 & & & & $4 / 26 / 90$ & $<$ & 10000.0 \\
\hline & I Ethylene oxide & PPB & 10.0 & & $11 / 03 / 89$ & $<$ & 10.0 \\
\hline & I Ethyleneimine & PPB & 10.0 & & $11 / 03 / 89$ & $<$ & 10.0 \\
\hline & | Ethylenethiourea & PPB & 200.0 & & $11 / 03 / 89$ & $<$ & 200.0 \\
\hline & I Fluoranthene & PPB & 10.0 & & | 11/03/89 & $<$ & 10.0 \\
\hline & I Fluorene & PPB & 10.0 & & | 11/03/89 & $<$ & 10.0 \\
\hline & I Fluoride & PPB & 500.0 & 4000.0 & 1 5/30/89 & $<$ & 500.0 \\
\hline & 1 & & & & | 11/03/89 & $<$ & 500.0 \\
\hline
\end{tabular}




\section{WHC-MR. 0229}

$11 / 26 / 90$

Geosciences Group PARADOX Database

Well Result Report

1100 Area Groundwater Monitoring

\begin{tabular}{|c|c|c|c|c|c|c|c|c|c|}
\hline WELL & \multicolumn{5}{|c|}{ CONSTITUENT } & 1 & \multicolumn{3}{|c|}{ RESULT } \\
\hline Name & $\begin{array}{l}1 \\
1\end{array}$ & Name & Units & $\begin{array}{l}\text { Detection } \\
\text { Limit }\end{array}$ & $\begin{array}{l}\text { Drinking Water } \\
\text { Standard }\end{array}$ & 1 & $\begin{array}{l}\text { Sample } \\
\text { Date }\end{array}$ & & $\begin{array}{l}\text { nalys is } \\
\text { Value }\end{array}$ \\
\hline 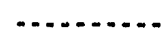 & 1 & ............ & $\cdots+. .$. & ............ & $\ldots$ & 1 & ......... & - & \\
\hline 6-540-E14 & 1 & Fluoride & PPB & 500.0 & 4000.0 & i & $1 / 04 / 90$ & $<$ & 500.0 \\
\hline & 1 & & & & & 1 & $2 / 09 / 90$ & $<$ & 500.0 \\
\hline & 1 & & & & & 1 & $2 / 09 / 90$ & $<$ & 500.0 \\
\hline & 1 & & & & & i & $3 / 20 / 90$ & $<$ & 500.0 \\
\hline & i & & & & & i & $4 / 26 / 90$ & $<$ & 500.0 \\
\hline & 1 & Formal in & PPB & 500.0 & & $i$ & $11 / 03 / 89$ & $<$ & 500.0 \\
\hline & $i$ & Gamma-BHC & PPB & .1 & 4.0 & i & $5 / 30 / 89$ & $<$ & .1 \\
\hline & I & & & & & i & $11 / 03 / 89$ & $<$ & .1 \\
\hline & 1 & & & & & 1 & $2 / 09 / 90$ & $<$ & .1 \\
\hline & 1 & & & & & 1 & $2 / 09 / 90$ & $<$ & .1 \\
\hline & 1 & Gross beta & $\mathrm{PCI} / \mathrm{L}$ & 8.0 & 50.0 & 1 & $5 / 30 / 89$ & & 3.0 \\
\hline & 1 & & & & & 1 & $11 / 03 / 89$ & $<$ & 1.9 \\
\hline & 1 & & & & & i & $2 / 09 / 90$ & $<$ & .4 \\
\hline & 1 & & & & & i & $2 / 09 / 90$ & & 3.0 \\
\hline & i & Heptachlor & PPB & .1 & .4 & 1 & $11 / 03 / 89$ & $<$ & .1 \\
\hline & I & Heptchlor epoxide & PPB & .1 & & i & $11 / 03 / 89$ & $<$ & .1 \\
\hline & 1 & Hexachlorobenzene & PPB & 10.0 & & 1 & $5 / 30 / 89$ & $<$ & 10.0 \\
\hline & 1 & & & & & i & $11 / 03 / 89$ & $<$ & 10.0 \\
\hline & 1 & & & & & 1 & $2 / 09 / 90$ & $<$ & 10.0 \\
\hline & 1 & Hexachlor obutadi ene & PPB & 10.0 & & i & $11 / 03 / 89$ & $<$ & 10.0 \\
\hline & 1 & Hexach lorocyclopentadi ene & PPB & 10.0 & & 1 & $11 / 03 / 89$ & $<$ & 10.0 \\
\hline & 1 & Hexachloroethane & PPB & 10.0 & & 1 & $11 / 03 / 89$ & $<$ & 10.0 \\
\hline & 1 & Hexachlorophene & PPB & 10.0 & & 1 & $5 / 30 / 89$ & $<$ & 10.0 \\
\hline & 1 & & & & & i & $11 / 03 / 89$ & $<$ & 10.0 \\
\hline & 1 & & & & & 1 & $2 / 09 / 90$ & $<$ & 10.0 \\
\hline & 1 & Hexachloropropene & PPB & 10.0 & & i & $11 / 03 / 89$ & $<$ & 10.0 \\
\hline & I & Hydrazine, Low Detection Level & PPB & 30.0 & & 1 & $11 / 03 / 89$ & $<$ & 30.0 \\
\hline & 1 & Indsno $(1,2,3-c d)$ pyrene & PPB & 10.0 & & 1 & $11 / 03 / 89$ & $<$ & 10.0 \\
\hline & 1 & lodomethane & PPB & 10.0 & & i & $11 / 03 / 89$ & $<$ & 10.0 \\
\hline & I & Iron & PPB & 30.0 & 300.0 & 1 & $11 / 03 / 89$ & & 394.0 \\
\hline & 1 & & & & & 1 & $1 / 04 / 90$ & & 67.0 \\
\hline & I & & & & & 1 & $2 / 09 / 90$ & & 44.0 \\
\hline & 1 & & & & & 1 & $2 / 09 / 90$ & & 58.0 \\
\hline & 1 & & & & & ! & $3 / 20 / 90$ & & 65.0 \\
\hline & i & Iron, filtered & PPB & 30.0 & 300.0 & 1 & $5 / 30 / 89$ & $<$ & 30.0 \\
\hline & 1 & & & & & 1 & $11 / 03 / 89$ & $<$ & 30.0 \\
\hline & 1 & & & & & 1 & $1 / 04 / 90$ & $<$ & 30.0 \\
\hline & 1 & & & & & I & $2 / 09 / 90$ & $<$ & 30.0 \\
\hline & 1 & & & & & 1 & $2 / 09 / 90$ & $<$ & 30.0 \\
\hline & I & & & & & I & $3 / 20 / 90$ & $<$ & 30.0 \\
\hline & i & Isobutyl alcohol & PPB & 10000.0 & & 1 & $11 / 03 / 89$ & $<$ & 10000.0 \\
\hline & 1 & Isodrin & PPB & 10.0 & & i & $11 / 03 / 89$ & $<$ & 10.0 \\
\hline & 1 & Isophorone & PPB & 10.0 & & 1 & $11 / 03 / 89$ & $<$ & 10.0 \\
\hline & 1 & Isosafrole & PPB & 10.0 & & 1 & $11 / 03 / 89$ & $<$ & 10.0 \\
\hline
\end{tabular}


WHC-MR. 0229

$11 / 26 / 90$

Geosciences Group PARADOX Database

Well Result Report

1100 Area Groundwater Monitoring

\begin{tabular}{|c|c|c|c|c|c|c|c|c|}
\hline \multirow{2}{*}{$\begin{array}{l}\text { WELL } \\
\text { Name }\end{array}$} & \multicolumn{4}{|c|}{ CONSTITUENT } & 1 & \multicolumn{3}{|c|}{ RESULT } \\
\hline & Name & Units & $\begin{array}{c}\text { Detection } \\
\text { Limit }\end{array}$ & $\begin{array}{l}\text { Drinking Water } \\
\text { Standard }\end{array}$ & 1 & $\begin{array}{l}\text { Sample } \\
\text { Date }\end{array}$ & & $\begin{array}{l}\text { inalysis } \\
\text { Value }\end{array}$ \\
\hline (........... & | & -...... & -......... & - & 1 & -........ & $\cdots$ & ......... \\
\hline \multirow{44}{*}{$6-540-E 14$} & I Kepone & PPB & 1.0 & & 1 & $11 / 03 / 09$ & $<$ & 9.0 \\
\hline & I Kerosene & PPB & 10000.0 & & I & $5 / 30 / 89$ & $<$ & 10000.0 \\
\hline & 1 & & & & i & $11 / 03 / 89$ & $<$ & 10000.0 \\
\hline & i & & & & i & $2 / 09 / 90$ & $<$ & 10000.0 \\
\hline & I Lead (graphite furnace) & PPB & 5.0 & 50.0 & i & $11 / 03 / 89$ & $<$ & 5.0 \\
\hline & 1 & & & & 1 & $1 / 04 / 90$ & $<$ & 5.0 \\
\hline & 1 & & & & 1 & $2 / 09 / 90$ & $<$ & 5.0 \\
\hline & 1 & & & & 1 & $2 / 09 / 90$ & $<$ & 5.0 \\
\hline & 1 & & & & i & $3 / 20 / 90$ & $<$ & 5.0 \\
\hline & I Lead, filtered & PPB & 5.0 & 50.0 & 1 & $5 / 30 / 89$ & $<$ & 5.0 \\
\hline & 1 & & & & 1 & $11 / 03 / 89$ & $<$ & 5.0 \\
\hline & 1 & & & & 1 & $1 / 04 / 90$ & $<$ & 5.0 \\
\hline & I & & & & 1 & $2 / 09 / 90$ & $<$ & 5.0 \\
\hline & 1 & & & & 1 & $2 / 09 / 90$ & $<$ & 5.0 \\
\hline & 1 & & & & 1 & $3 / 20 / 90$ & $<$ & 5.0 \\
\hline & I Lithium & PPB & 10.0 & & i & $11 / 03 / 89$ & $<$ & 10.0 \\
\hline & 1 & & & & 1 & $1 / 04 / 90$ & $<$ & 10.0 \\
\hline & 1 & & & & 1 & $2 / 09 / 90$ & $<$ & 10.0 \\
\hline & 1 & & & & I & $2 / 09 / 90$ & $<$ & 10.0 \\
\hline & 1 & & & & 1 & $3 / 20 / 90$ & $<$ & 10.0 \\
\hline & I Lithium, filtered & PPB & 10.0 & & 1 & $5 / 30 / 89$ & $<$ & 10.0 \\
\hline & 1 & & & & 1 & $11 / 03 / 89$ & $<$ & 10.0 \\
\hline & I & & & & 1 & $1 / 04 / 90$ & $<$ & 10.0 \\
\hline & 1 & & & & 1 & $2 / 09 / 90$ & $<$ & 10.0 \\
\hline & 1 & & & & 1 & $2 / 09 / 90$ & $<$ & 10.0 \\
\hline & 1 & & & & 1 & $3 / 20 / 90$ & • & 10.0 \\
\hline & I Magnesium & PPB & 50.0 & & 1 & $11 / 03 / 89$ & & 4840.0 \\
\hline & 1 & & & . & 1 & $1 / 04 / 90$ & & 4210.0 \\
\hline & 1 & & & & 1 & $2 / 09 / 90$ & & 4900.0 \\
\hline & 1 & & & & 1 & $2 / 09 / 90$ & & 4910.0 \\
\hline & 1 & & & & 1 & $3 / 20 / 90$ & & 4940.0 \\
\hline & I Magnesium, filtered & PPB & 50.0 & & 1 & $5 / 30 / 89$ & & 3970.0 \\
\hline & 1 & & & & 1 & $11 / 03 / 89$ & & 4840.0 \\
\hline & 1 & & & & 1 & $1 / 04 / 90$ & & 4430.0 \\
\hline & 1 & & & & 1 & $2 / 09 / 90$ & & 4890.0 \\
\hline & 1 & & & & 1 & $2 / 09 / 90$ & & 4920.0 \\
\hline & 1 & & & & 1 & $3 / 20 / 90$ & & 4810.0 \\
\hline & I Maleic hydrizide & PPB & 500.0 & & 1 & $11 / 03 / 89$ & $<$ & 500.0 \\
\hline & I Malononitrile & PPB & 10.0 & & 1 & $11 / 03 / 89$ & $<$ & 10.0 \\
\hline & I Manganese & PPB & 5.0 & 50.0 & I & $11 / 03 / 89$ & $<$ & 5.0 \\
\hline & I & & & & i & $1 / 04 / 90$ & $<$ & 5.0 \\
\hline & 1 & & & & 1 & $2 / 09 / 90$ & $<$ & 5.0 \\
\hline & 1 & & & & 1 & $2 / 09 / 90$ & $<$ & 5.0 \\
\hline & 1 & & & & 1 & $3 / 20 / 90$ & $<$ & 5.0 \\
\hline
\end{tabular}




\section{WHC-MR- 0229}

$11 / 26 / 90$

Geosciences Group PARADOX Dutabase

Well Result Report

1100 Area Groundwater Monitoring

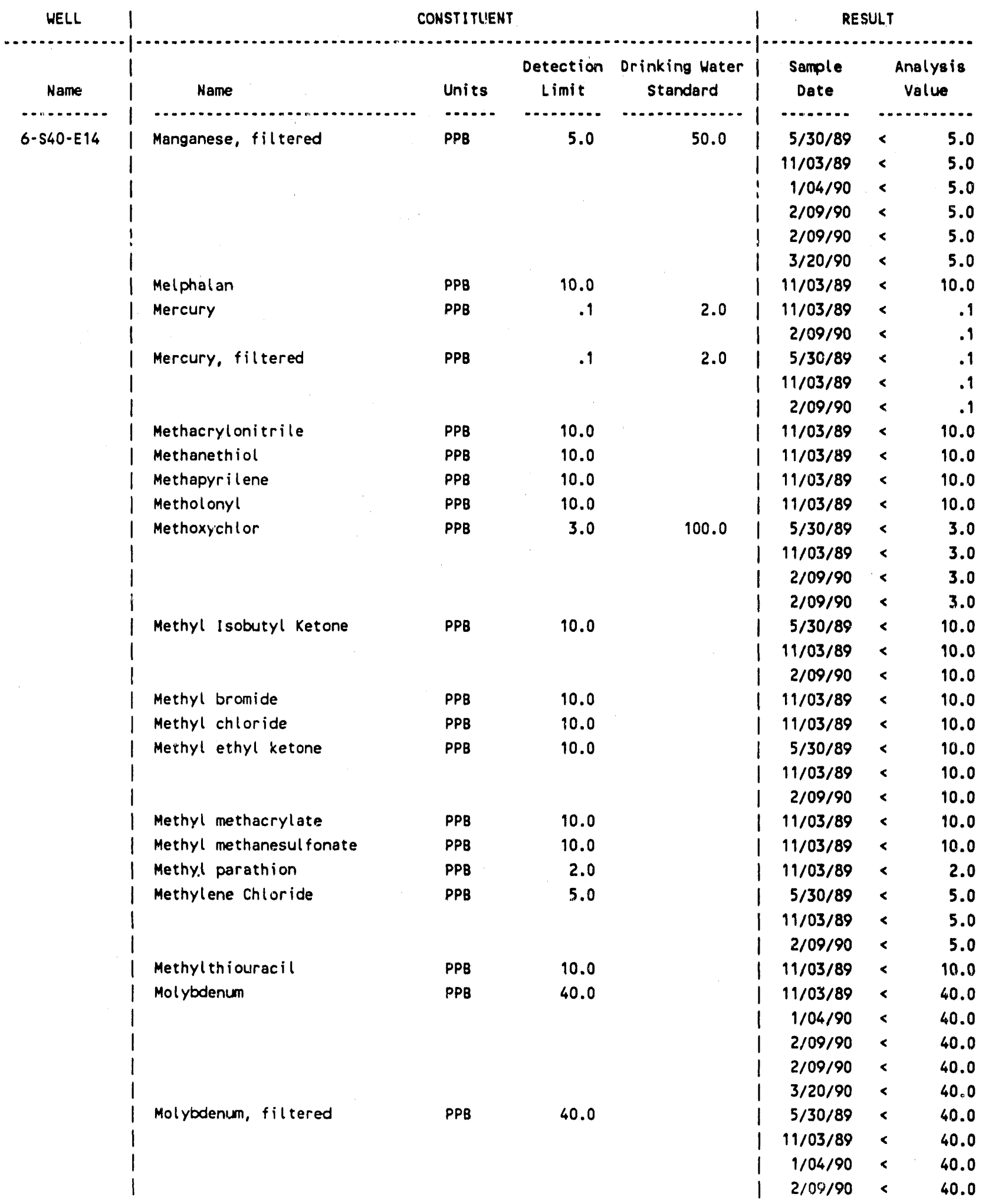


WHC-MR. 0229

$11 / 26 / 90$

Geosciences Group PARADOX Database

Well Result Report

Page 36

1100 Area Groundwater Monitoring

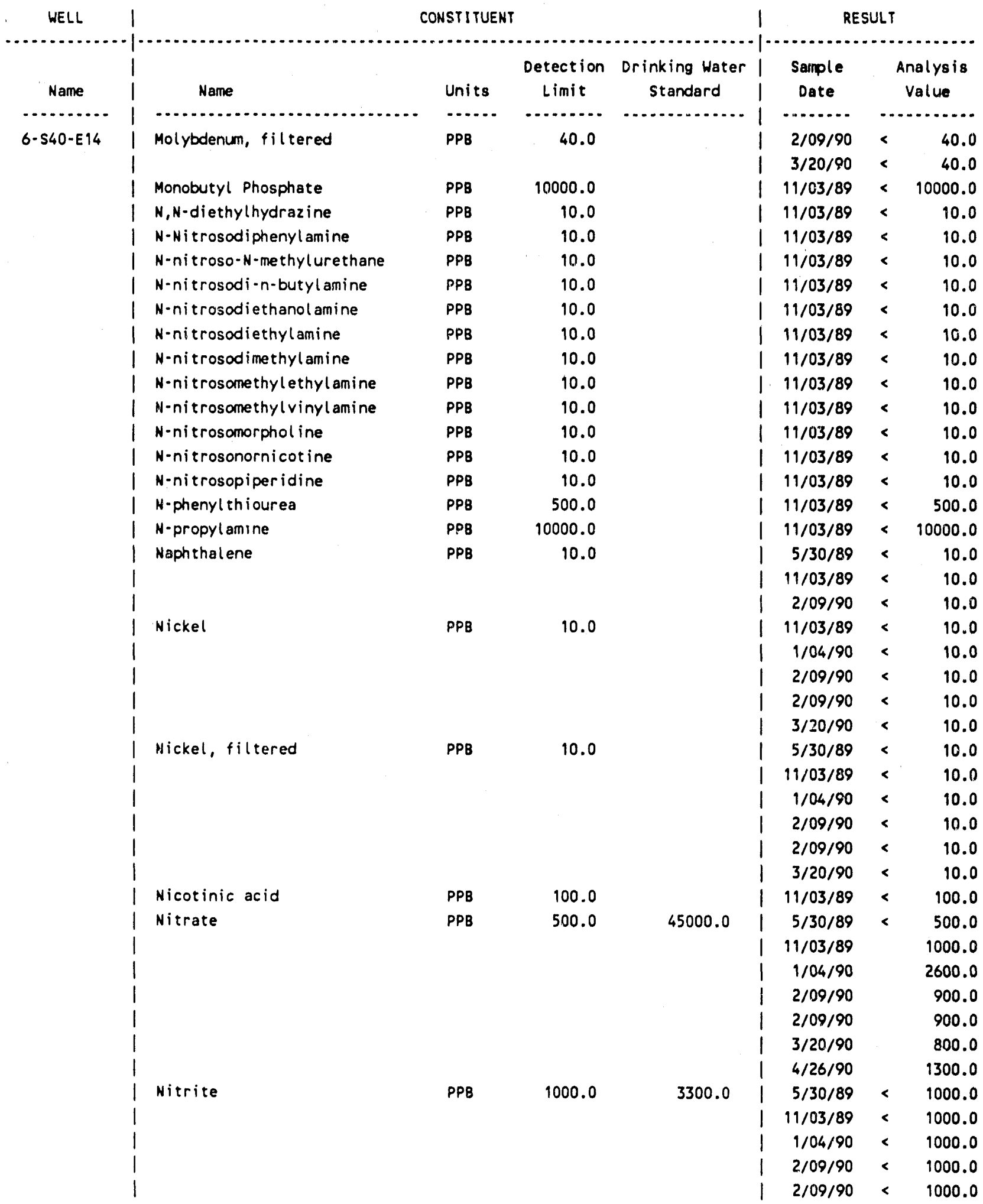


WHC-MR- 0229

$11 / 26 / 90$

Geosciences Group PARADOX Databese

Well Result Report

1100 Area Groundwater Monitoring

\begin{tabular}{|c|c|c|c|c|c|c|c|c|}
\hline WELL & 1 & & NSTITUE & & & 1 & & ULT \\
\hline Name: & 1 & Name & Units & $\begin{array}{l}\text { Detection } \\
\text { Limit }\end{array}$ & $\begin{array}{l}\text { Orinking Water } \\
\text { standard }\end{array}$ & 1 & $\begin{array}{l}\text { Sample } \\
\text { Date }\end{array}$ & $\begin{array}{l}\text { Analysis } \\
\text { Value }\end{array}$ \\
\hline$\cdots$ & 1 & - & ...... & $\ldots \ldots \ldots$ & $\cdots+\ldots, n$ & 1 & ......... & ............. \\
\hline $6-540-E 14$ & 1 & Nitrite & PPB & 1000.0 & 3300.0 & 1 & $3 / 20 / 90$ & $<\quad 1000.0$ \\
\hline & 1 & & & & & 1 & $4 / 26 / 90$ & 1000.0 \\
\hline & 1 & Nitrobenzine & PPB & 10.0 & & 1 & $11 / 03 / 89$ & 10.0 \\
\hline & 1 & Nitrosopyrrolidine & PPB & 10.0 & & 1 & $11 / 03 / 89$ & 10.0 \\
\hline & 1 & $0,0,0-$ triethyl phosphorothioat & PPB & 10.0 & & 1 & $11 / 03 / 89$ & 10.0 \\
\hline & 1 & 0 - toluidine hydrochloride & PPB & 10.0 & & 1 & $11 / 03 / 89$ & 10.0 \\
\hline & 1 & $P$ benzoquinone & PPB & 10.0 & & 1 & $11 / 03 / 89$ & 10.0 \\
\hline & 1 & P-chloro-m-cresol & PPB & 10.0 & & 1 & $11 / 03 / 89$ & 10.0 \\
\hline & 1 & P-chloroaniline & PPB & 10.0 & & 1 & $11 / 03 / 89$ & 10.0 \\
\hline & 1 & P-dimethylaminoazobenzene & PPB & 10.0 & & 1 & $11 / 03 / 89$ & 10.0 \\
\hline & 1 & P-nitroaniline & PPB & 10.0 & & 1 & $\$ 1 / 03 / 89$ & 10.0 \\
\hline & 1 & PHORATE & PPB & - 2.0 & & 1 & $11 / 03 / 89$ & 2.0 \\
\hline & 1 & Paraldehyde & PPB & 10000.0 & & 1 & $11 / 03 / 89$ & 10000.0 \\
\hline & 1 & Parathion & PPB & 2.0 & & I & $11 / 03 / 89$ & 2.0 \\
\hline & 1 & Pcdd's & PPB & .0 & & i & $11 / 03 / 89$ & .0 \\
\hline & 1 & Pcdf's & PPB & .0 & & 1 & $11 / 03 / 89$ & .0 \\
\hline & 1 & Pentachlorobenzene & PPB & 10.0 & & I & $5 / 30 / 89$ & 10.0 \\
\hline & 1 & & & & & 1 & $11 / 03 / 89$ & 10.0 \\
\hline & 1 & & & & & 1 & $2 / 09 / 90$ & 10.0 \\
\hline & 1 & Pentachloroe thane & PPB & 10.0 & & 1 & $11 / 03 / 89$ & 10.0 \\
\hline & 1 & Pentachloroni trobenzene & PPB & 10.0 & & i & $11 / 03 / 89$ & 10.0 \\
\hline & 1 & Pentachlorophenol & PPB & 50.0 & 200.0 & 1 & $11 / 03 / 89$ & 50.0 \\
\hline & 1 & Perchlorate & PPB & 500.0 & & 1 & $11 / 03 / 89$ & 500.0 \\
\hline & 1 & Phenacet in & PPB & 10.0 & & 1 & $11 / 03 / 89$ & 10.0 \\
\hline & 1 & Phenanthrene & PPB & 10.0 & & 1 & $11 / 03 / 89$ & 10.0 \\
\hline & 1 & Phenol & PPB & 10.0 & & 1 & $5 / 30 / 89$ & 10.0 \\
\hline & 1 & & & & & 1 & $11 / 03 / 89$ & 10.0 \\
\hline & 1 & & & & & 1 & $2 / 09 / 90$ & 10.0 \\
\hline & 1 & Phenyl enediamine & PPB & 10.0 & & 1 & $11 / 03 / 89$ & 10.0 \\
\hline & 1 & Phosphate & PPB & 1000.0 & & I & $5 / 30 / 89$ & 1000.0 \\
\hline & 1 & & & & & 1 & $11 / 03 / 89$ & 1000.0 \\
\hline & 1 & & & & . & 1 & $1 / 04 / 90$ & 1000.0 \\
\hline & 1 & & & & & 1 & $2 / 09 / 90$ & 1000.0 \\
\hline & 1 & & & & & 1 & $2 / 09 / 90$ & 1000.0 \\
\hline & 1 & & & & & 1 & $3 / 20 / 90$ & 1000.0 \\
\hline & 1 & & & & & 1 & $4 / 26 / 90$ & 1000.0 \\
\hline & 1 & Phthalic acid esters & PPB & 10.0 & & 1 & $11 / 03 / 89$ & 10.0 \\
\hline & I & Potassium & PPB & 100.0 & & 1 & $11 / 03 / 89$ & 1200.0 \\
\hline & 1 & & & & & 1 & $1 / 04 / 90$ & 710.0 \\
\hline & 1 & & & & & 1 & $2 / 09 / 90$ & 789.0 \\
\hline & 1 & & & & & I & $2 / 09 / 90$ & 799.0 \\
\hline & 1 & & & & & I & $3 / 20 / 90$ & 765.0 \\
\hline & 1 & Potassium, filtered & PPB & 100.0 & & 1 & $5 / 30 / 89$ & 977.0 \\
\hline & 1 & & & & & | & $11 / 03 / 89$ & 1960.0 \\
\hline
\end{tabular}


WHC-MR. 0229

$11 / 26 / 90$

Geosciences Group PARADOX Database

Well Result Report

1100 Area Groundwater Monitoring

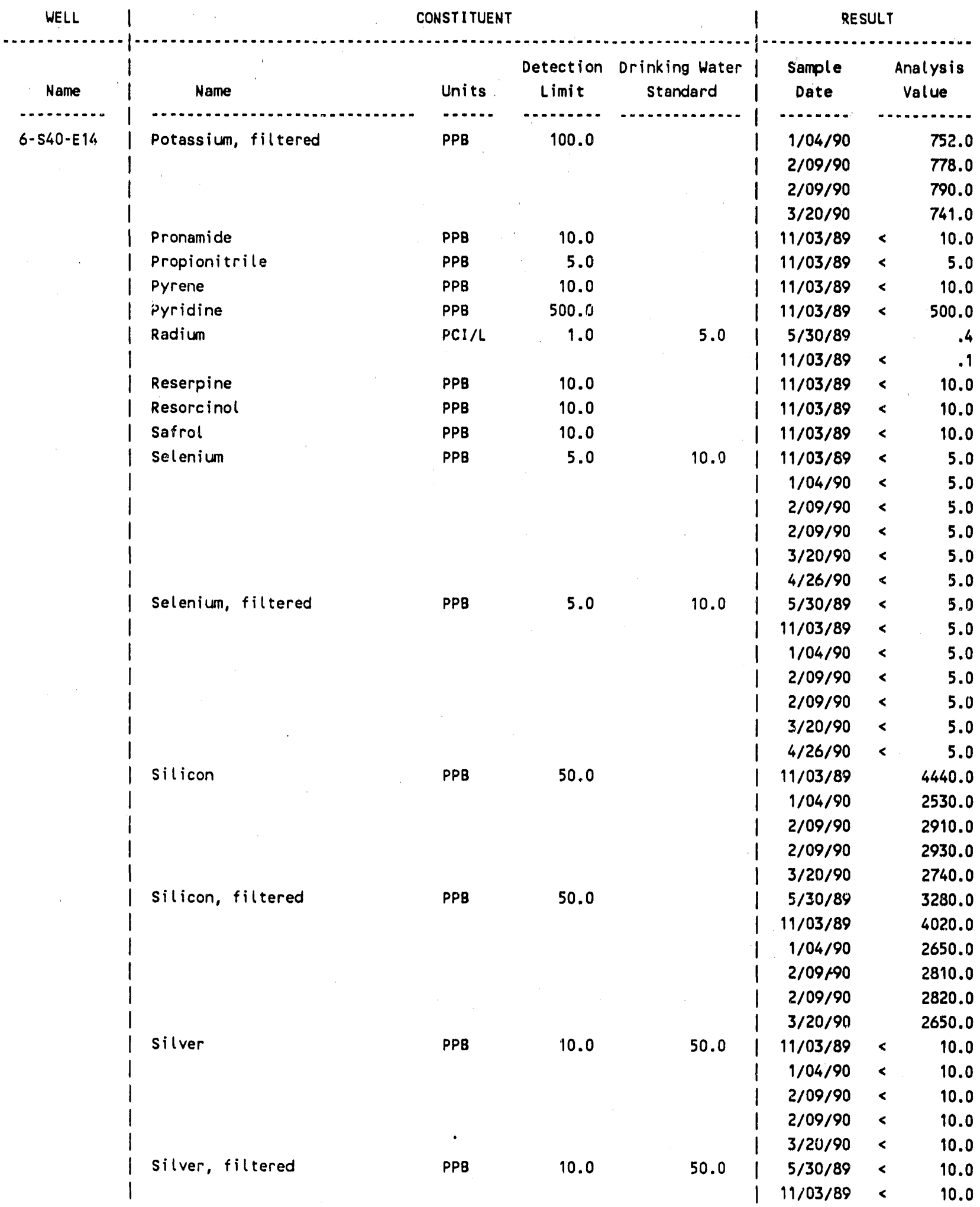




\section{WHC-MR. 0229}

$11 / 26 / 90$

Geosciences Group PARADOX Database

Well Result Report

1100 Area Groundwater Monitoring

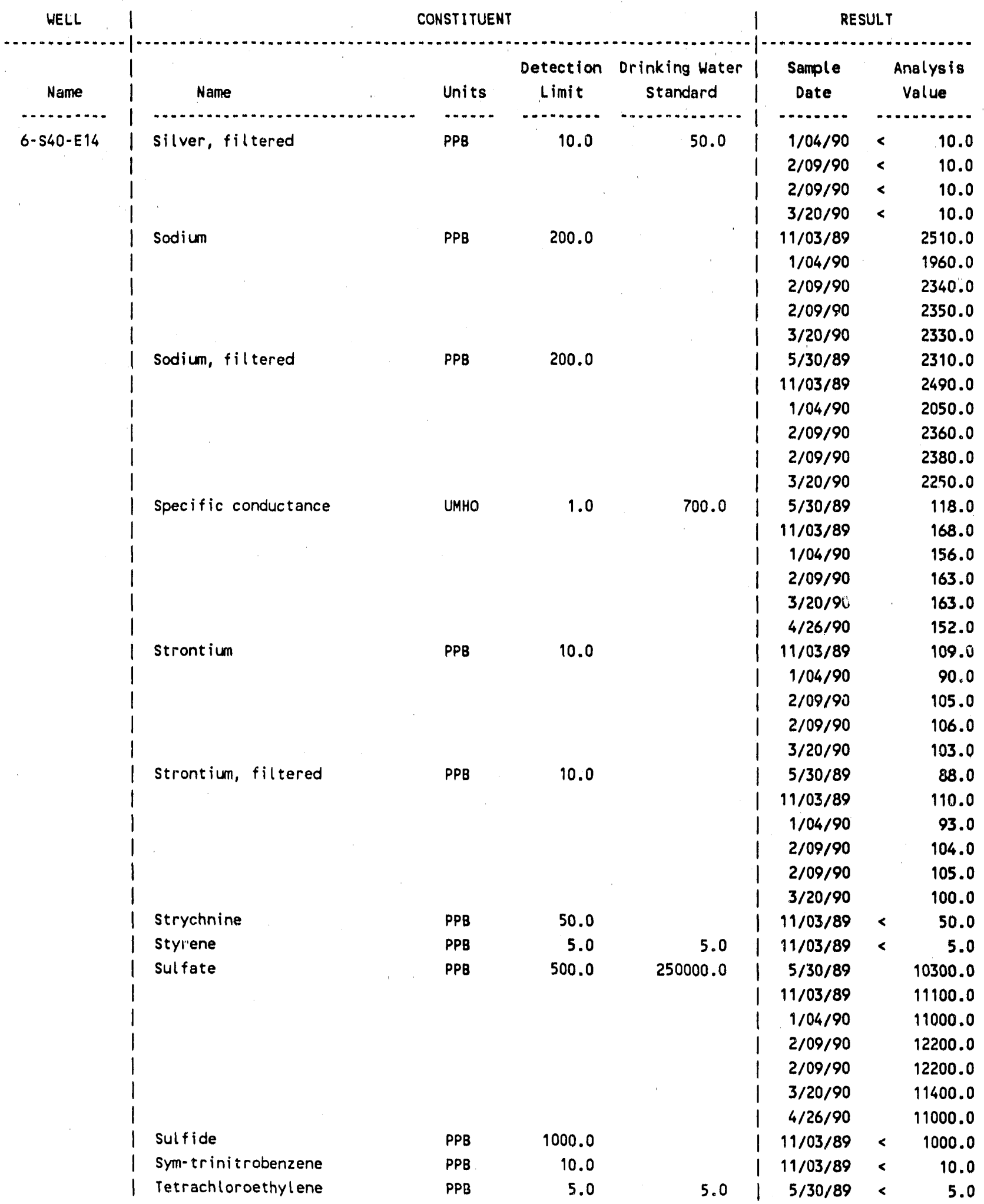


WHC-MR- 0229

$11 / 26 / 90$

Geosciences Group PARADOX Database

Page 40

Well Result Report

1100 Area Groundwater Monitoring

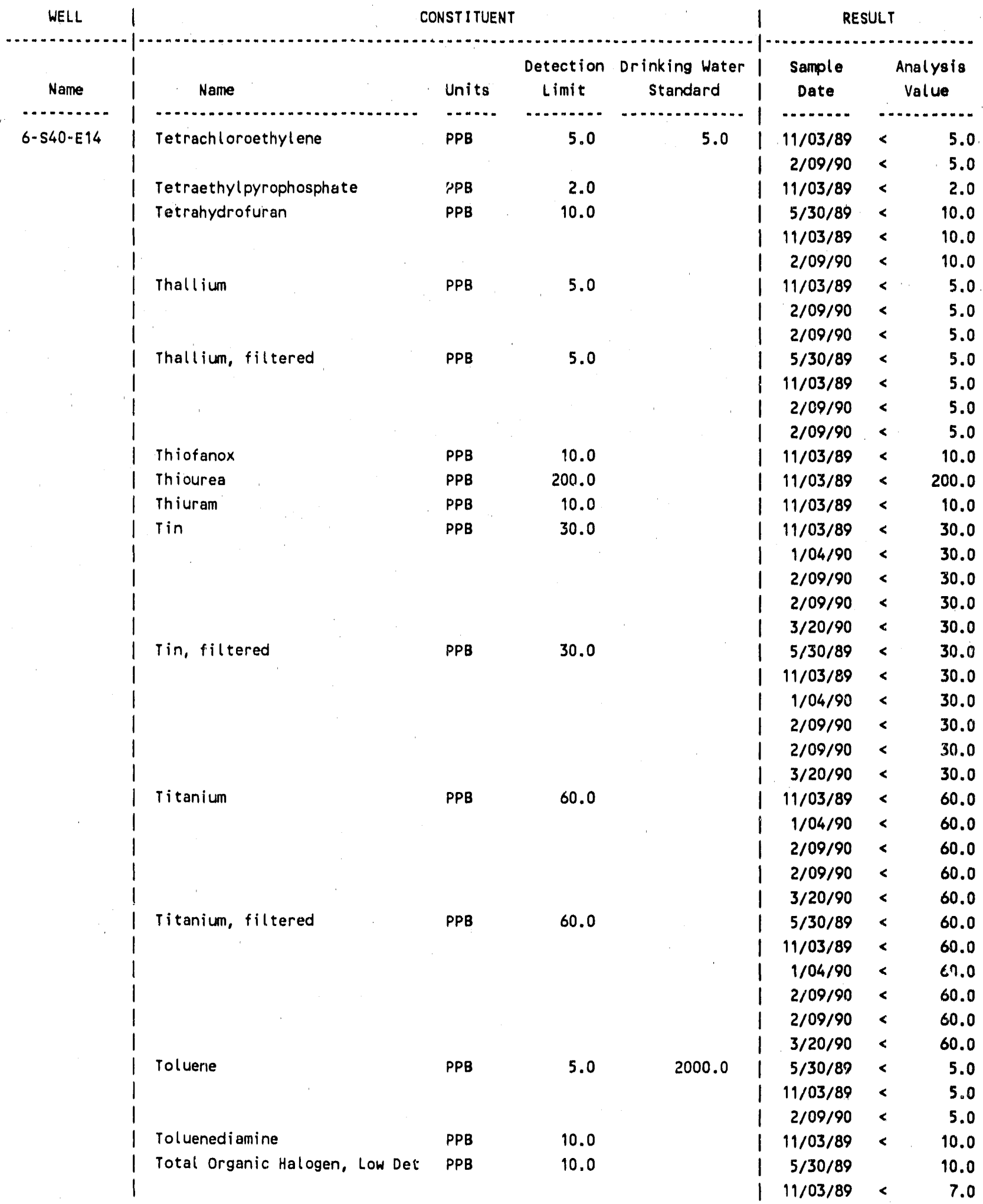


WHC-MR. 0229

$11 / 26 / 90$

Geosciences Group PARADOX Database

Well Result Report

Page 41

1100 Area Groundwater Monitoring

\begin{tabular}{|c|c|c|c|c|c|c|c|}
\hline \multirow{2}{*}{$\begin{array}{l}\text { WELL } \\
\text { Name }\end{array}$} & 1 & \multicolumn{3}{|c|}{ ONSTITUENT } & \multicolumn{3}{|c|}{ RESULT } \\
\hline & Name & Units & $\begin{array}{l}\text { Detection } \\
\text { Limit }\end{array}$ & $\begin{array}{l}\text { Drinking Water } \\
\text { standard }\end{array}$ & $\begin{array}{l}\text { Sample } \\
\text { Dete }\end{array}$ & & $\begin{array}{l}\text { inalys is } \\
\text { Value }\end{array}$ \\
\hline a.......... & | & …... & -.......... & n............... & 1 $\cdots \ldots . .$. & & \\
\hline \multirow[t]{44}{*}{$6-540-E 14$} & I Total Organic Halogen, Low Det & PPB & 10.0 & & $2 / 09 / 90$ & & 16.0 \\
\hline & I Total carbon & PPB & 1000.0 & & $5 / 30 / 89$ & & 15100.0 \\
\hline & 1 & & & & $11 / 03 / 89$ & & 18200.0 \\
\hline & I & & & & $2 / 09 / 90$ & & 17200.0 \\
\hline & I Total organic carbon & PPB & 1000.0 & & $5 / 30 / 89$ & $<$ & 900.0 \\
\hline & 1 & & & & | 11/03/89 & $<$ & 800.0 \\
\hline & 1 & & & & $2 / 09 / 90$ & $<$ & 800.0 \\
\hline & I Toxaphene & PPB & 1.0 & 5.0 & $5 / 30 / 89$ & $<$ & 1.0 \\
\hline & 1 & & & & | 11/03/89 & $<$ & 1.0 \\
\hline & i & & & & I $2 / 09 / 90$ & $<$ & 1.0 \\
\hline & 1 & & & & $2 / 09 / 90$ & $<$ & 1.0 \\
\hline & | Trans-1,2-dichloroethene & PPB & 5.0 & 70.0 & $5 / 30 / 89$ & $<$ & 5.0 \\
\hline & I & & & & I 11/03/89 & $<$ & 5.0 \\
\hline & 1 & & & & | $2 / 09 / 90$ & $<$ & 5.0 \\
\hline & I Tributylphosphoric Acid & PPB & 10.0 & & $5 / 30 / 89$ & $<$ & 10.0 \\
\hline & 1 & & & & | $11 / 03 / 89$ & 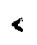 & 10.0 \\
\hline & 1 & & & & $1 \quad 2 / 09 / 90$ & $<$ & 10.0 \\
\hline & I Trichloroethylene & PPB & 5.0 & 5.0 & $5 / 30 / 89$ & $<$ & 5.0 \\
\hline & 1 & & & & | 1i/03/89 & $<$ & 5.0 \\
\hline & 1 & & & & $2 / 09 / 90$ & $<$ & 5.0 \\
\hline & I Trichloromethanethiol & PPB & 10.0 & & | 11/03/89 & $<$ & 10.0 \\
\hline & I Trichloromonof luoromethane & PPB & 10.0 & & $11 / 03 / 89$ & $<$ & 10.0 \\
\hline & I Tris(2,3-dibromopropyl) phosph & PPB & 10.0 & & $11 / 03 / 89$ & $<$ & 10.0 \\
\hline & I Tritium & PCI/L & 500.0 & 20000.0 & I $5 / 30 / 89$ & $<$ & 66.1 \\
\hline & I & & & & | 11/03/89 & $<$ & 101.0 \\
\hline & I Turbidity & NTU & .1 & 5.0 & $11 / 03 / 89$ & & 4.7 \\
\hline & 1 & & & & $2 / 09 / 90$ & & .2 \\
\hline & I Vanadium & PPB & 5.0 & & | 11/03/89 & $<$ & 5.0 \\
\hline & 1 & & & & $1 / 04 / 90$ & $<$ & 5.0 \\
\hline & 1 & & & & $2 / 09 / 90$ & $<$ & 5.0 \\
\hline & 1 & & & & $2 / 09 / 90$ & $<$ & 5.0 \\
\hline & 1 & & & & $3 / 20 / 90$ & $<$ & 5.0 \\
\hline & I Vanadium, filtered & PPB & 5.0 & & 1 $5 / 30 / 89$ & & 7.0 \\
\hline & 1 & & & & I 11/03/89 & $<$ & 5.0 \\
\hline & 1 & & & & $1 \quad 1 / 04 / 90$ & $<$ & 5.0 \\
\hline & 1 & & & & $2 / 09 / 90$ & $<$ & 5.0 \\
\hline & 1 & & & & $2 / 09 / 90$ & $<$ & 5.0 \\
\hline & 1 & & & & $1 \quad 3 / 20 / 90$ & s & 5.0 \\
\hline & I Vinyl Acetate & PPB & 5.0 & & I 11/03/89 & $<$ & 5.0 \\
\hline & I Vinyl chloride & PPB & 10.0 & 2.0 & $5 / 30 / 89$ & $<$ & 10.0 \\
\hline & 1 & & & & | 11/03/89 & $<$ & 10.0 \\
\hline & 1 & & & & $2 / 09 / 90$ & $<$ & 10.0 \\
\hline & 1 Warfarin & PPB & 10.0 & & | 11/03/89 & $<$ & 10.0 \\
\hline & I xylene-m & PPB & 5.0 & 10000.0 & $1 \quad 5 / 30 / 89$ & $<$ & 5.0 \\
\hline
\end{tabular}


WHC-MR. 0229

Geosciences Group PARADOX Database

$11 / 26 / 90$

$\longrightarrow$ Page 42

Well Result Report

1.100 Area Groundwater Monitoring

\begin{tabular}{|c|c|c|c|c|c|c|c|}
\hline WELL & \multicolumn{4}{|c|}{ CONSTI I TUENT } & \multicolumn{3}{|c|}{ RESULT } \\
\hline Name & Name & Units & $\begin{array}{l}\text { Detection } \\
\text { Limit }\end{array}$ & $\begin{array}{l}\text { Orinking Water } \\
\text { Standard }\end{array}$ & $\begin{array}{c}\text { Sample } \\
\text { Date }\end{array}$ & & $\begin{array}{l}\text { Analysis } \\
\text { Value }\end{array}$ \\
\hline .............. & 1 & ........ & .......... & ……… & ( $\ldots \ldots$ & & \\
\hline $6-540-E 14$ & I xylene- $m$ & PPB & 5.0 & 10000.0 & $11 / 03 / 89$ & $<$ & 5.0 \\
\hline & i & & & & $2 / 09 / 90$ & $<$ & 5.0 \\
\hline & I xylene-0,p & PPB & 5.0 & 10000.0 & $5 / 30 / 89$ & $<$ & 5.0 \\
\hline & 1 & & & & $11 / 03 / 89$ & $<$ & 5.0 \\
\hline & 1 & & & & $2 / 09 / 90$ & $<$ & 5.0 \\
\hline & I zinc & PPB & 5.0 & 5000.0 & $11 / 03 / 89$ & & 12.0 \\
\hline & 1 & & & & $1 / 04 / 90$ & $<$ & 5.0 \\
\hline & 1 & & & & $2 / 09 / 90$ & & 5.0 \\
\hline & 1 & & & & $2 / 09 / 90$ & $<$ & 5.0 \\
\hline & 1 & & & & $3 / 20 / 90$ & $<$ & 5.0 \\
\hline & I zinc, filtered & PPB & 5.0 & 5000.0 & $5 / 30 / 89$ & $<$ & 5.0 \\
\hline & 1 & & & & $111 / 03 / 89$ & $<$ & 5.0 \\
\hline & 1 & & & & $1 \quad 1 / 04 / 90$ & $<$ & 5.0 \\
\hline & 1 & & & & $2 / 09 / 90$ & $<$ & 5.0 \\
\hline & 1 & & & & $2 / 09 / 90$ & $<$ & 5.0 \\
\hline & 1 & & & & $3 / 20 / 90$ & $<$ & 5.0 \\
\hline & | zirconium & PPB & 50.0 & & $111 / 03 / 89$ & $<$ & 50.0 \\
\hline & 1 & & & & $1 / 04 / 90$ & $<$ & 50.0 \\
\hline & 1 & & & & $2 / 09 / 90$ & $<$ & 50.0 \\
\hline & i & & & & $2 / 09 / 90$ & $<$ & 50.0 \\
\hline & 1 & & & & $3 / 20 / 90$ & $<$ & 50.0 \\
\hline & I zirconium, filtered & PPB & 50.0 & & $5 / 30 / 89$ & $<$ & $50 . ?$ \\
\hline & 1 & & & & I 11/03/89 & $<$ & 50.0 \\
\hline & 1 & & & & $1 / 04 / 90$ & $<$ & 50.0 \\
\hline & 1 & & & & $2 / 09 / 90$ & $<$ & 50.0 \\
\hline & 1 & & & & $2 / 09 / 90$ & $<$ & 50.0 \\
\hline & 1 & & & & $3 / 20 / 90$ & $<$ & 50.0 \\
\hline & I dibromochlor ome thane & PPB & 5.0 & 100.0 & | 11/03/89 & $<$ & 5.0 \\
\hline & I m-Nitroaniline & PPB & $10 . \%$ & & $111 / 03 / 89$ & $<$ & 10.0 \\
\hline & I o-Nitroaniline & PPB & 14.0 & & $11 / 03 / 89$ & $<$ & 10.0 \\
\hline & I o-Nitrophenol & PPB & 10.0 & & I 11/03/89 & $<$ & 10.0 \\
\hline & | p-Dichlorobenzene & PPB & 5.0 & 750.0 & $5 / 30 / 89$ & $<$ & 5.0 \\
\hline & 1 & & & & $11 / 03 / 89$ & $<$ & 5.0 \\
\hline & 1 & & & & 1 $2 / 09 / 90$ & $<$ & 5.0 \\
\hline & 1 & & 10.0 & 750.0 & $5 / 30 / 89$ & $<$ & 10.0 \\
\hline & 1 & & & & I 11/03/89 & $<$ & 10.0 \\
\hline & 1 & & & & $2 / 09 / 90$ & $<$ & 10.0 \\
\hline & I p-Nitrophenol & PPB & 10.0 & & $111 / 03 / 89$ & $<$ & 10.0 \\
\hline & I pH, Field Measurement & & .1 & 8.5 & i $5 / 30 / 89$ & & 7.6 \\
\hline & 1 & & & & I 11/03/89 & & 8.1 \\
\hline & | & & & & $1 \quad 1 / 04 / 90$ & & 8.2 \\
\hline & 1 & & & & $2 / 09 / 90$ & & 8.0 \\
\hline & 1 & & & & $3 / 20 / 90$ & & 7.9 \\
\hline & 1 & & & & $4 / 26 / 90$ & & 7.9 \\
\hline
\end{tabular}


WHC-MR. $\quad 0 \approx 29$

$11 / 26 / 90$

Geosciences Group PARADOX Database

Well Result Report

1100 Area Groundwater Monitoring

\begin{tabular}{|c|c|c|c|c|c|c|c|}
\hline WELL & 1 & NSTITUE & & & 1 & ULT & \\
\hline Name & Name & inits & $\begin{array}{l}\text { Detection } \\
\text { Limit }\end{array}$ & $\begin{array}{c}\text { Drinking Water } \\
\text { standard }\end{array}$ & $\begin{array}{l}\text { Sample } \\
\text { Date }\end{array}$ & & $\begin{array}{l}\text { Analysis } \\
\text { Value }\end{array}$ \\
\hline & | $\quad \ldots \ldots \ldots \ldots \ldots \ldots$ & ....... & (........... & (n............... & ( & & \\
\hline $6-540-E 14$ & I pH, Laboratory Measurement & & .0 & 8.5 & $5 / 30 / 89$ & & 7.8 \\
\hline & 1 & & & & $11 / 03 / 89$ & & 7.9 \\
\hline & 1 & & & & $2 / 09 / 90$ & & 7.6 \\
\hline & 1 & & & & 1 & & \\
\hline $6-341-E 13 A$ & | 0,0-Diethyl-0,2-pyrazinyl phos & PPB & 10.0 & & $11 / 03 / 89$ & $<$ & 10.0 \\
\hline & | 1,1,1,2-tetrachlorethane & PPB & 10.0 & & | 11/03/89 & $<$ & 10.0 \\
\hline & | 1,1,1-trichloroethane & PPB & 5.0 & 200.0 & I $5 / 24 / 89$ & $<$ & 5.0 \\
\hline & 1 & & & & $5 / 30 / 89$ & $<$ & 5.0 \\
\hline & 1 & & & & I $11 / 03 / 89$ & $<$ & 5.0 \\
\hline & i & & & & $2 / 09 / 90$ & $<$ & 5.0 \\
\hline & | 1,1,2,2-tetrachlorethane & PPB & 5.0 & & $11 / 03 / 89$ & $<$ & 5.0 \\
\hline & | 1,1,2-trichloroethane & PPB & 5.0 & & $5 / 24 / 89$ & $<$ & 5.0 \\
\hline & 1 & & & & $5 / 30 / 89$ & $<$ & 5.0 \\
\hline & 1 & & & & $11 / 03 / 89$ & $<$ & 5.0 \\
\hline & 1 & & & & $2 / 09 / 90$ & $<$ & 5.0 \\
\hline & | 1,1-dichloroethane & PPB & 5.0 & & $5 / 24 / 89$ & $<$ & 5.0 \\
\hline & 1 & & & & $5 / 30 / 89$ & $<$ & 5.0 \\
\hline & 1. & & & & $11 / 03 / 89$ & $<$ & 5.0 \\
\hline & 1 & & & & $12 / 09 / 90$ & $<$ & 5.0 \\
\hline & | 1,1-dichloroethylene & PPB & 10.0 & 7.0 & $11 / 03 / 89$ & $<$ & 10.0 \\
\hline & | 1,1-dimethylhydrazine & PPB & 10.0 & & | 11/03/89 & $<$ & 10.0 \\
\hline & | 1,2,3,4-tetrachlorobenzene & PPB & 10.0 & & $15 / 30 / 89$ & $<$ & 10.0 \\
\hline & 1 & & & & $11 / 03 / 89$ & $<$ & 10.0 \\
\hline & 1 & & & & $2 / 09 / 90$ & $<$ & 10.0 \\
\hline & I 1,2,3,5-tetrachlorobenzene & PPB & 10.0 & & $5 / 30 / 89$ & $<$ & 10.0 \\
\hline & 1 & & & & $11 / 03 / 89$ & $<$ & 10.0 \\
\hline & 1 & & & & | $2 / 09 / 90$ & $<$ & 10.0 \\
\hline & | 1,2,3-trichlorobenzene & PPB & 10.0 & & $5 / 30 / 89$ & $<$ & 10.0 \\
\hline & 1 & & & & | $11 / 03 / 89$ & $<$ & 10.0 \\
\hline & 1 & & & & $2 / 09 / 90$ & $<$ & 10.0 \\
\hline & | 1,2,3-trichloropropane & PPB & 10.0 & & | 11/03/89 & $<$ & 10.0 \\
\hline & | 1,2,4,5-tetrachlorobenzene & PPB & 10.0 & & $5 / 30 / 89$ & $<$ & 10.0 \\
\hline & 1 & & & & 1. $11 / 03 / 89$ & $<$ & 10.0 \\
\hline & 1 & & & & $1 \quad 2,09 / 90$ & $<$ & 10.0 \\
\hline & 1,2,4-trichlorobenzene & PPB & 10.0 & & $5 / 30 / 89$ & $<$ & 10.0 \\
\hline & 1 & & & & $11 / 03 / 89$ & $<$ & 10.0 \\
\hline & 1 & & & & $2 / 09 / 90$ & $<$ & 10.0 \\
\hline & | 1,2-dibramo-3-chloropropane & PPB & 10.0 & .2 & I 11/03/89 & $<$ & 10.0 \\
\hline & | 1,2-dibromoethane & PPB & 10.0 & & | 11/03/89 & $<$ & 10.0 \\
\hline & 1 1,2-dichlorobenzene & PPB & 10.0 & & $5 / 30 / 89$ & $<$ & 10.0 \\
\hline & 1 & & & & | 11/03/89 & $<$ & 10.0 \\
\hline & I & & & & 1 $2 / 09 / 90$ & $<$ & 10.0 \\
\hline & I 1,2-dichloroethane & PPB & 5.0 & 5.0 & $5 / 24 / 89$ & $<$ & 5.0 \\
\hline & 1 & & & & $5 / 30 / 89$ & $<$ & 5.0 \\
\hline
\end{tabular}




\section{WHC-MR. 0229}

Well Result Report

1100 Area Groundwater Monitoring

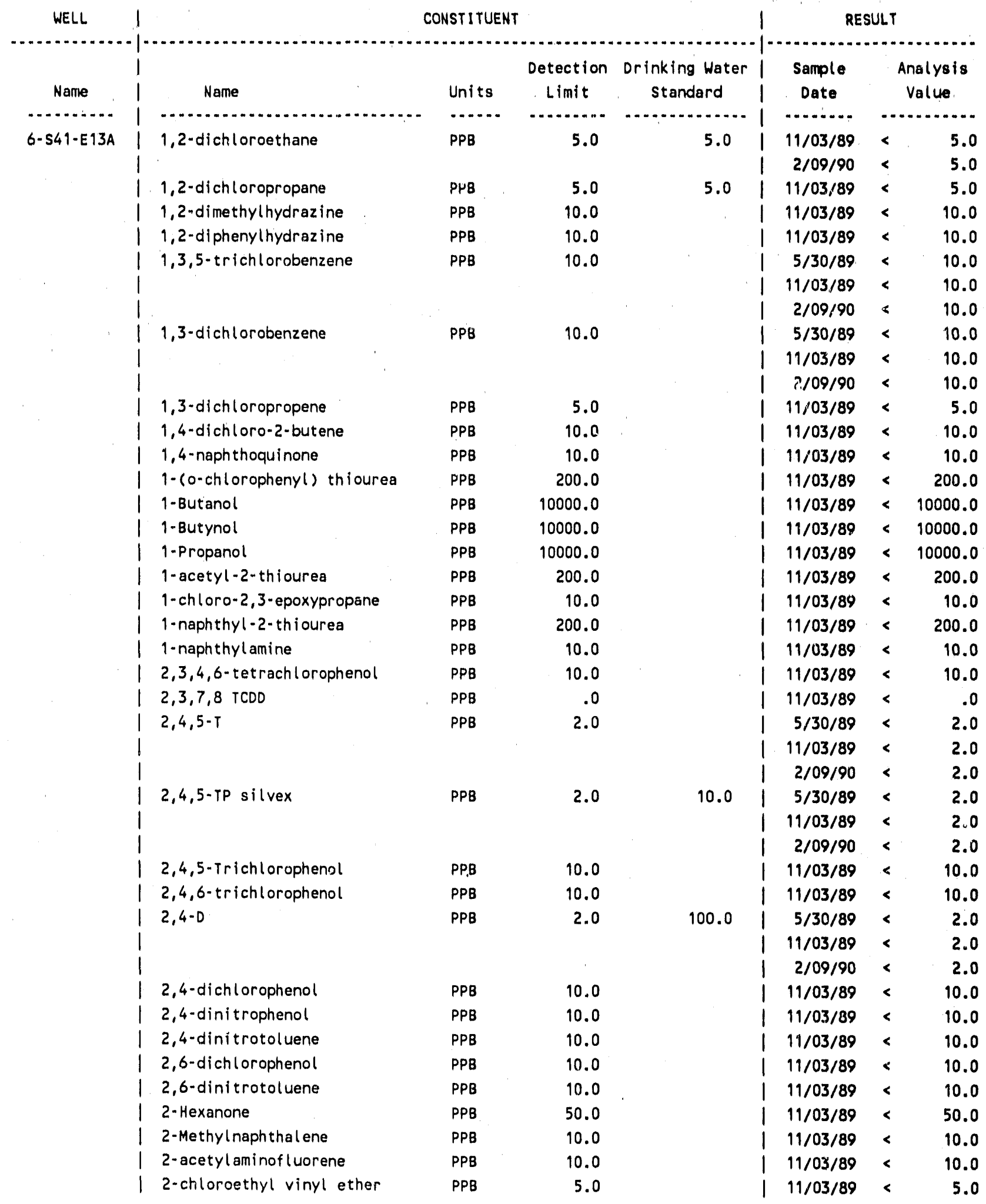


WHC-MR- 0229

Geosciences Group PARADOX Database

Well Result Report

1100 Area Groundwater Monitoring

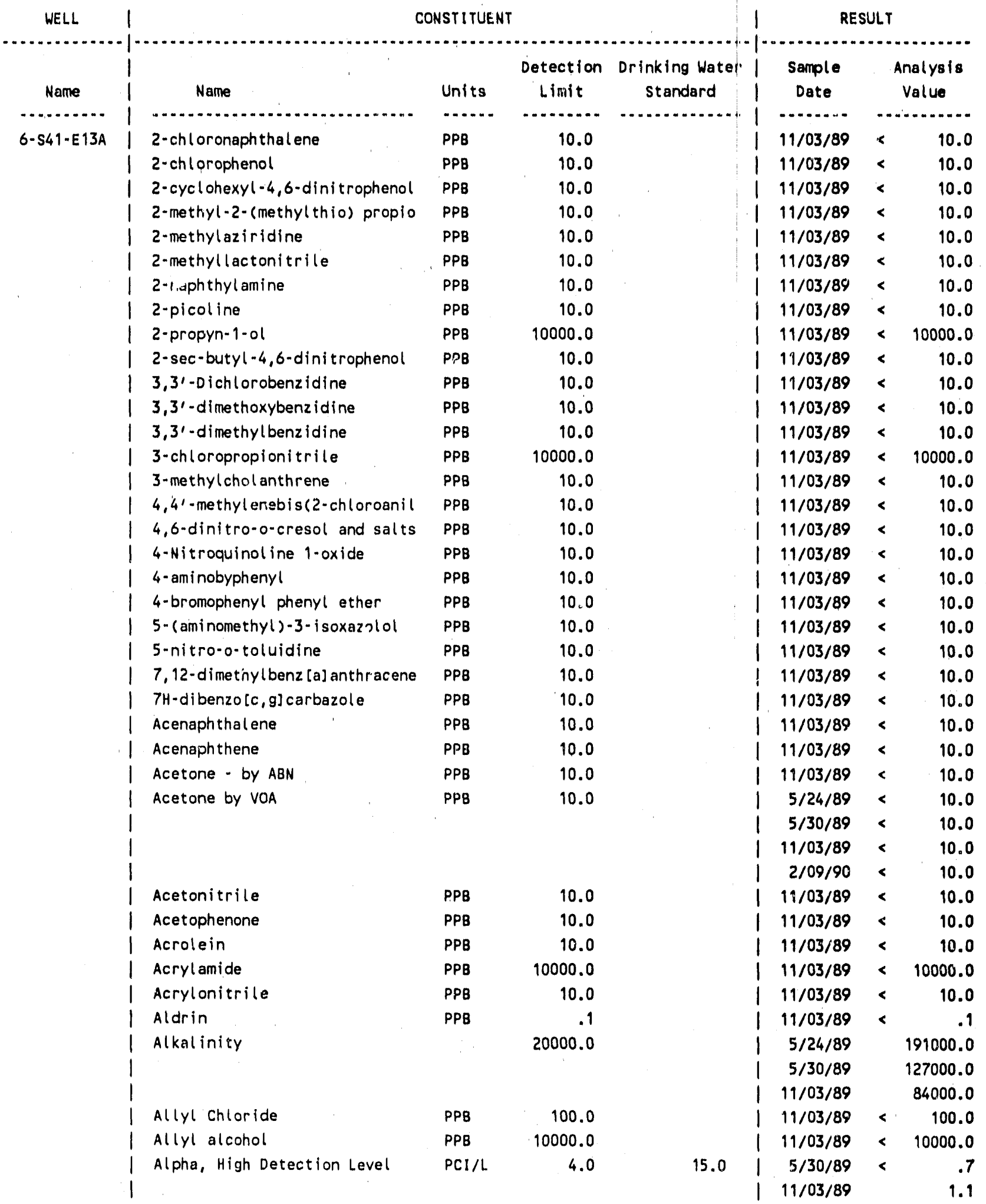




\section{WHC-MR. 0229}

$11 / 26 / 90$

Geosciences Group PARADOX Database

Well Result Report

1100 Area Groundwater Monitoring

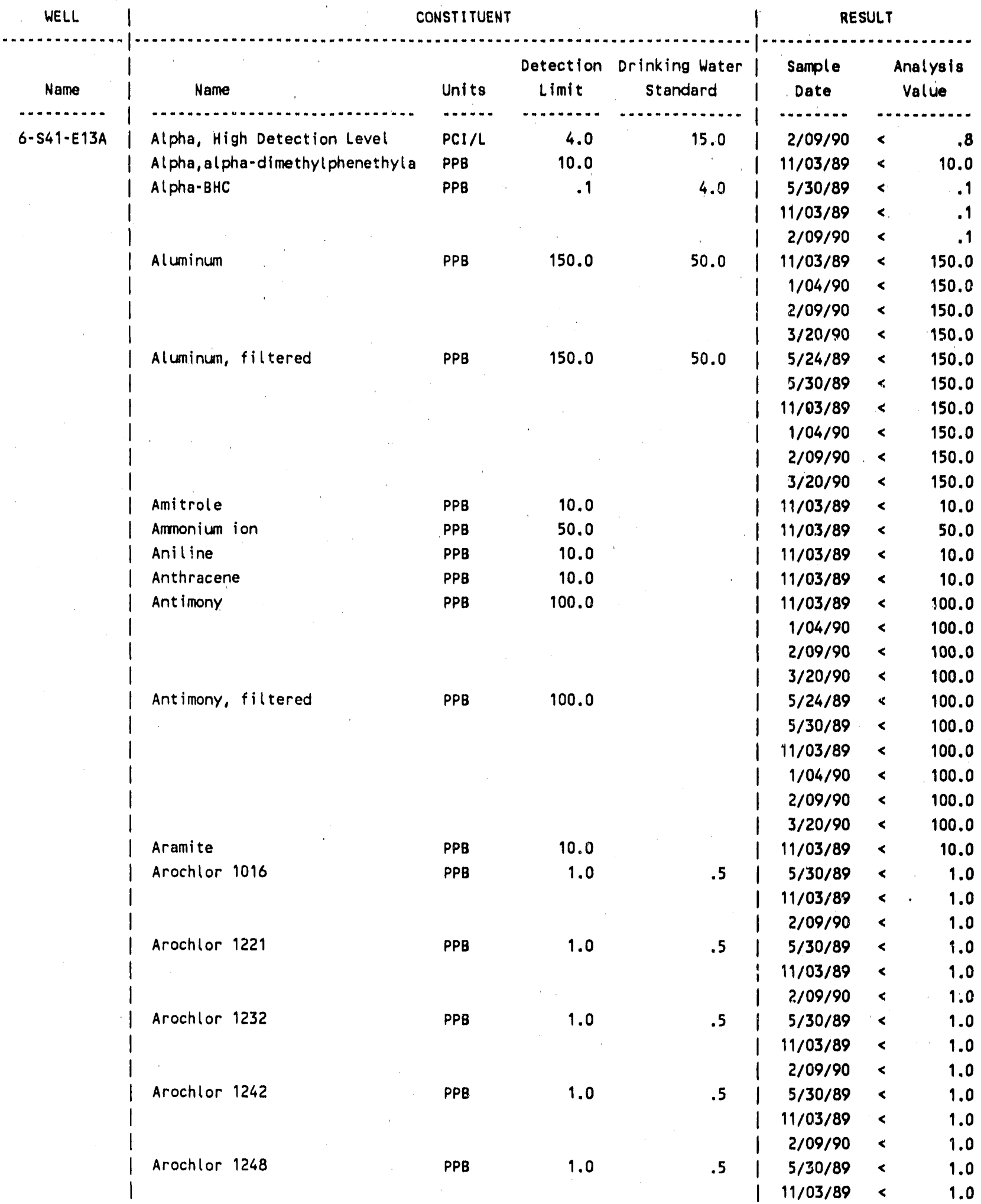


WHC-MR. 0229

$11 / 26 / 90$

Geosciences Group PARADOX Database

Well Result Report

Page 47

1100 Area Groundwater Monitoring

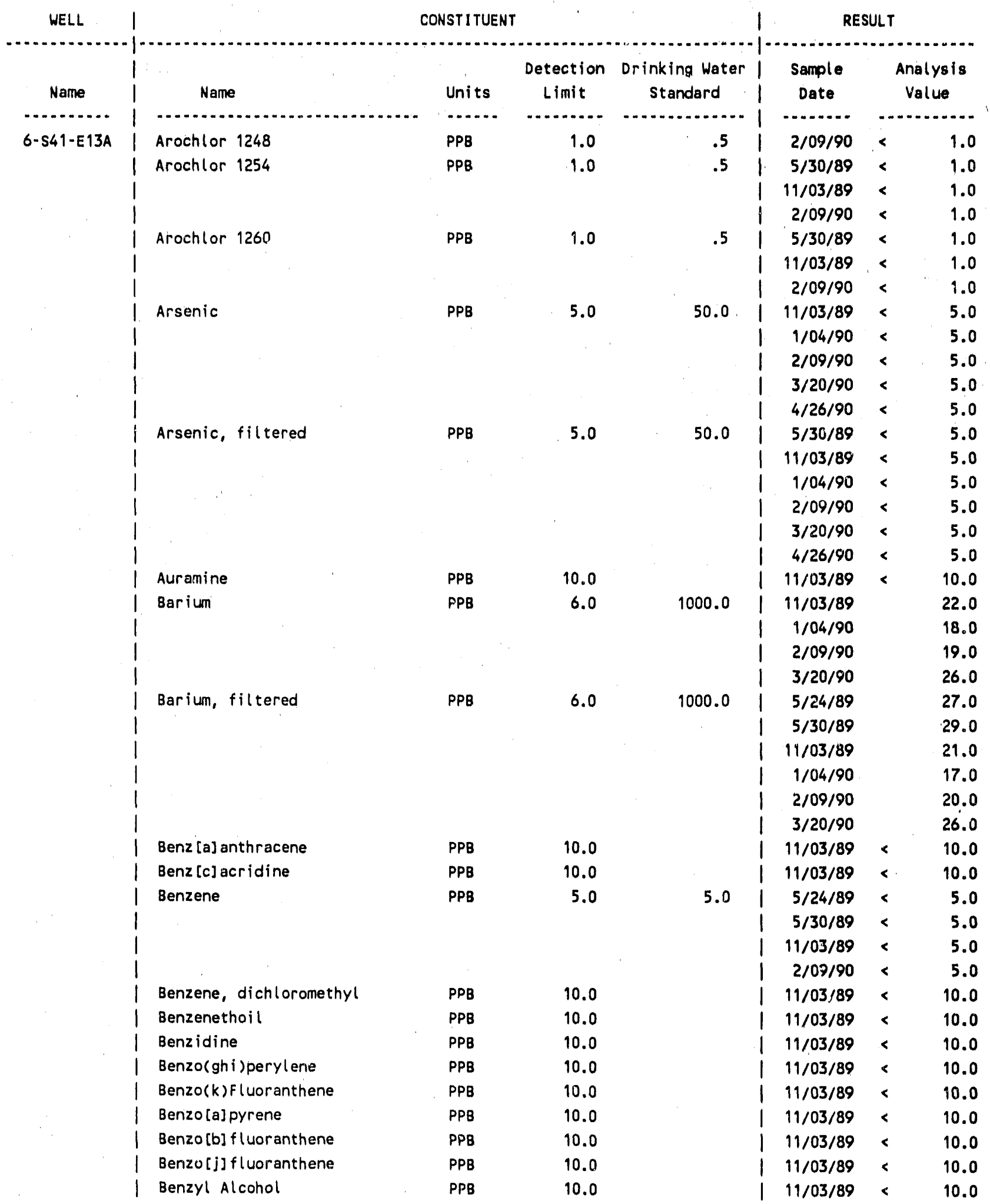


WHC-MR. 0229

$11 / 26 / 90$

Grosciences Group PARADOX Database

Well Result Report

Page 48

1100 Area Groundwater Monitoring

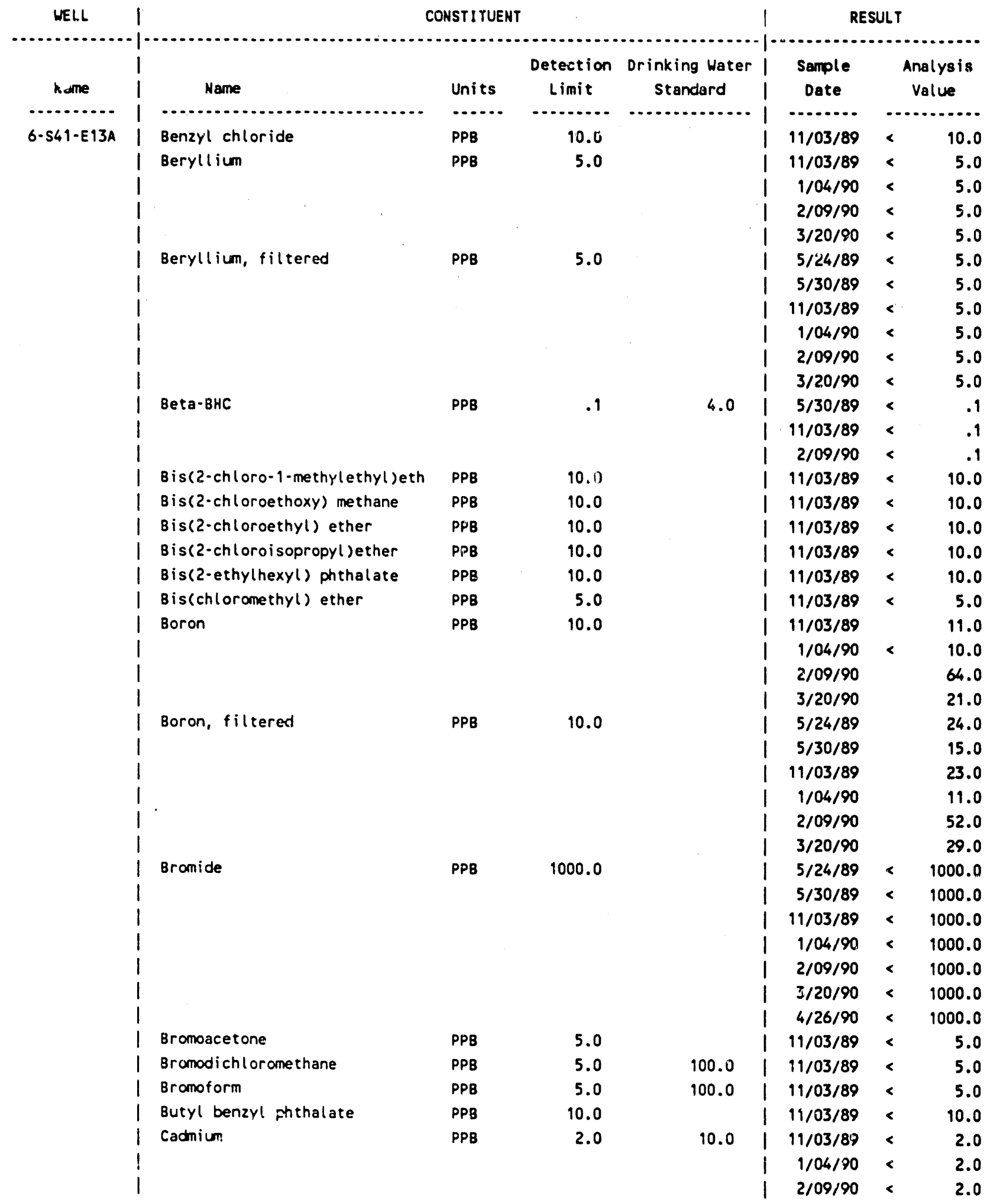




\section{WHC-MR. 0229}

Well Result Report

1100 Area Groundwater Monitoring

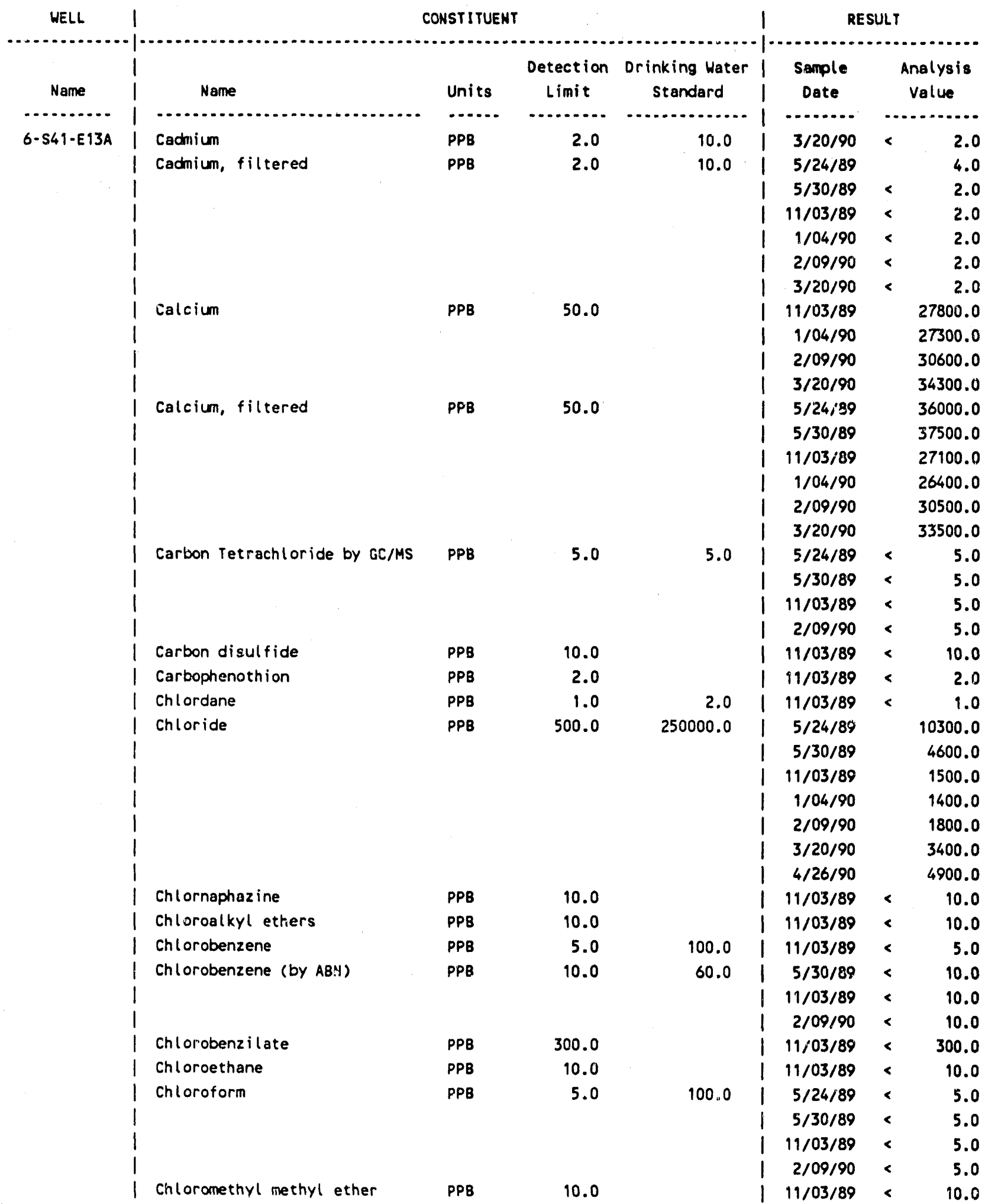


WHC-MR. 0229

$11 / 26 / 90$

Geosciences Group PARADOX Databose

Well Result Report

1100 Area Groundwater Monitoring

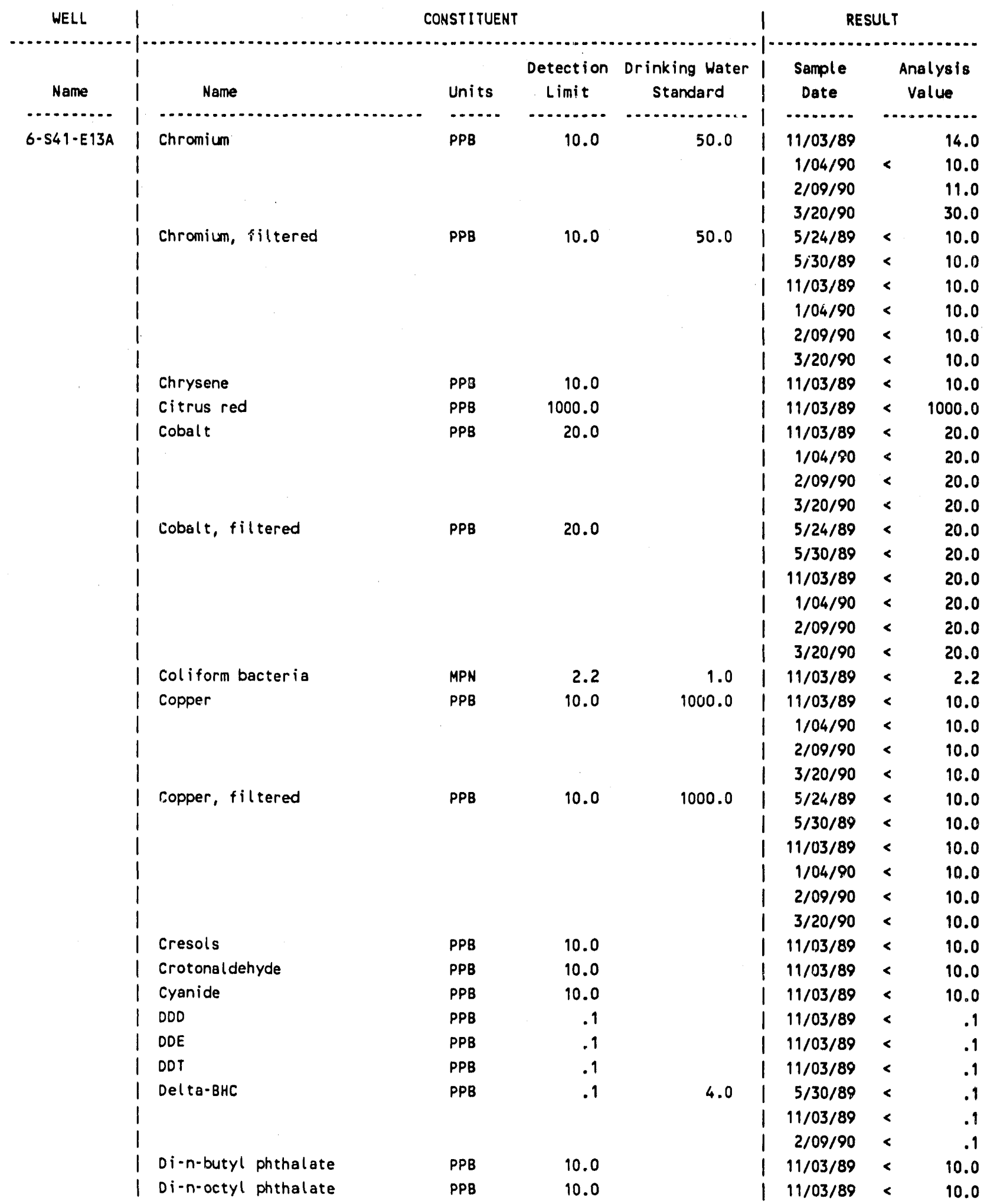




\section{WHC-MR- 0229}

Well Result Report

1100 Area Groundwater Monitoring

\begin{tabular}{|c|c|c|c|c|c|c|c|c|c|}
\hline WELL & 1 & \multicolumn{4}{|c|}{ CONSTI TUENT } & 1 & \multicolumn{3}{|c|}{ RESULT } \\
\hline Name & i & Name & Units & $\begin{array}{l}\text { Detection } \\
\text { Limit }\end{array}$ & $\begin{array}{c}\text { Drinking Water } \\
\text { standard }\end{array}$ & 1 & $\begin{array}{l}\text { Sample } \\
\text { Date }\end{array}$ & & $\begin{array}{l}\text { Analysis } \\
\text { Value }\end{array}$ \\
\hline -........... & $i$ & $\ldots \ldots \ldots \ldots \ldots$ & ...... & .......... & $\ldots \ldots \ldots \ldots$ & i & .......... & & .......... \\
\hline $6-541-E 13 A$ & $i$ & Di-n-propylnitrosamine & PPB & 10.0 & & $\mathrm{i}$ & $11 / 03 / 89$ & $<$ & 10.0 \\
\hline & 1 & Diallate & PPB & 10.0 & & I & $11 / 03 / 89$ & $<$ & 10.0 \\
\hline & $i$ & Dibenz $[a, h]$ acridine & PPB & 10.0 & & i & $11 / 03 / 89$ & $<$ & 10.0 \\
\hline & i & Dibenz $[a, h]$ anthracene & PPB & 10.0 & & i & $11 / 03 / 89$ & $<$ & 10.0 \\
\hline & 1 & Dibenz $[a, j]$ acridine & PPB & 10.0 & & i & $11 / 03 / 89$ & $<$ & 10.0 \\
\hline & 1 & Dibenzo[a, e] pyrene & PPB & 10.0 & & i & $11 / 03 / 89$ & $<$ & 10.0 \\
\hline & i & Dibenzo $[a, h]$ pyrene & PPB & 10.0 & & 1 & $11 / 03 / 89$ & $<$ & 10.0 \\
\hline & 1 & Dibenzo[a, i]pyrene & PPB & 10.0 & & i & $11 / 03 / 89$ & $<$ & 10.0 \\
\hline & 1 & Dibenzofuran & PPB & 10.0 & & i & $11 / 03 / 89$ & $<$ & 10.0 \\
\hline & $i$ & Dibromome thane & PPB & 10.0 & & i & $11 / 03 / 89$ & $<$ & 10.0 \\
\hline & $i$ & Dibutyl Phosphate & PPB & 10000.0 & & i & $11 / 03 / 89$ & $<$ & 10000.0 \\
\hline & 1 & Dichlorodifluorome thane & PPB & 10.0 & & 1 & $11 / 03 / 89$ & $<$ & 10.0 \\
\hline & $i$ & Dieldrin & PPB & .1 & & i & $11 / 03 / 89$ & $<$ & .1 \\
\hline & 1 & Diethyl phthalate & PPB & 10.0 & & i & $11 / 03 / 89$ & $<$ & 10.0 \\
\hline & $i$ & Diethylstilbesterol & PPB & 200.0 & & i & $11 / 03 / 89$ & $<$ & 200.0 \\
\hline & $i$ & Dihydrosafrole & PPB & 10.0 & & 1 & $11 / 03 / 89$ & $<$ & 10.0 \\
\hline & i & Dimethoate & PPB & 2.0 & & i & $11 / 03 / 89$ & $<$ & 2.0 \\
\hline & 1 & Dimethyl phthalate & PPB & 10.0 & & i & $11 / 03 / 89$ & $<$ & 10.0 \\
\hline & i & Dinitrobenzene & PPB & 10.0 & & 1 & $11 / 03 / 89$ & $<$ & 10.0 \\
\hline & $i$ & Dinoseb & PPB & 10.0 & & $i$ & $11 / 03 / 89$ & $<$ & 10.0 \\
\hline & 1 & Dioxane & PPB & 500.0 & & i & $11 / 03 / 89$ & $<$ & 500.0 \\
\hline & 1 & Diphenylamine & PPB & 10.0 & & i & $11 / 03 / 89$ & $<$ & 10.0 \\
\hline & $i$ & Disulfoton & PPB & 2.0 & & i & $11 / 03 / 89$ & $<$ & 2.0 \\
\hline & 1 & Endosul fan 1 & PPB & .9 & & i & $11 / 03 / 89$ & $<$ & .1 \\
\hline & 1 & Endosulfan $\|$ & PPB & .1 & & i & $11 / 03 / 89$ & $<$ & .1 \\
\hline & $i$ & Endosulfan Sulfate & PPB & .5 & & $i$ & $11 / 03 / 89$ & $<$ & .5 \\
\hline & i & Endr in & PPB & .1 & .2 & i & $5 / 30 / 89$ & $<$ & .1 \\
\hline & 1 & & & & & i & $11 / 03 / 89$ & $<$ & .1 \\
\hline & 1 & & & & & i & $2 / 09 / 90$ & $<$ & .1 \\
\hline & 1 & Ethanol & PPB & 10000.0 & & i & $11 / 03 / 89$ & $<$ & 10000.0 \\
\hline & 1 & Ethyt benzene & PPB & 5.0 & 700.0 & i & $11 / 03 / 89$ & $<$ & 5.0 \\
\hline & 1 & Ethyl carbamate & PPB & 10000.0 & & i & $11 / 03 / 89$ & $<$ & 10000.0 \\
\hline & i & Ethyl cyanide & PPB & 10000.0 & & i & $11 / 03 / 89$ & $<$ & 10000.0 \\
\hline & 1 & Ethyl methacrylate & PPB & 10.0 & & 1 & $11 / 03 / 89$ & $<$ & 10.0 \\
\hline & 1 & Ethyl methanesulfonate & PPB & 10.0 & & i & $11 / 03 / 89$ & $<$ & 10.0 \\
\hline & 1 & Ethylene Glycol & PPB & 10000.0 & & i & $11 / 03 / 89$ & $<$ & 10000.0 \\
\hline & 1 & Ethylene glycol & PPB & 10000.0 & & 1 & $5 / 30 / 89$ & $<$ & 10000.0 \\
\hline & 1 & & & & & i & $1 / 04 / 90$ & $<$ & 10000.0 \\
\hline & i & & & & & i & $2 / 09 / 90$ & $<$ & 10000.0 \\
\hline & 1 & & & & & 1 & $3 / 20 / 90$ & $<$ & 10000.0 \\
\hline & 1 & & & & & 1 & $4 / 26 / 90$ & $<$ & 10000.0 \\
\hline & 1 & Ethylene oxide & PPB & 10.0 & & i & $11 / 03 / 89$ & $<$ & 10.0 \\
\hline & $i$ & Ethyleneimine & PPB & 10.0 & & 1 & $11 / 03 / 89$ & $<$ & 10.0 \\
\hline & $i$ & Ethyl enethiourea & PPB & 200.0 & & 1 & $11 / 03 / 89$ & s & 200.0 \\
\hline
\end{tabular}


WHC-MR. 0229

$11 / 26 / 90$

Geosciences Group PARADOX Database

Well Result Report

1100 Area Groundwater Monitoring

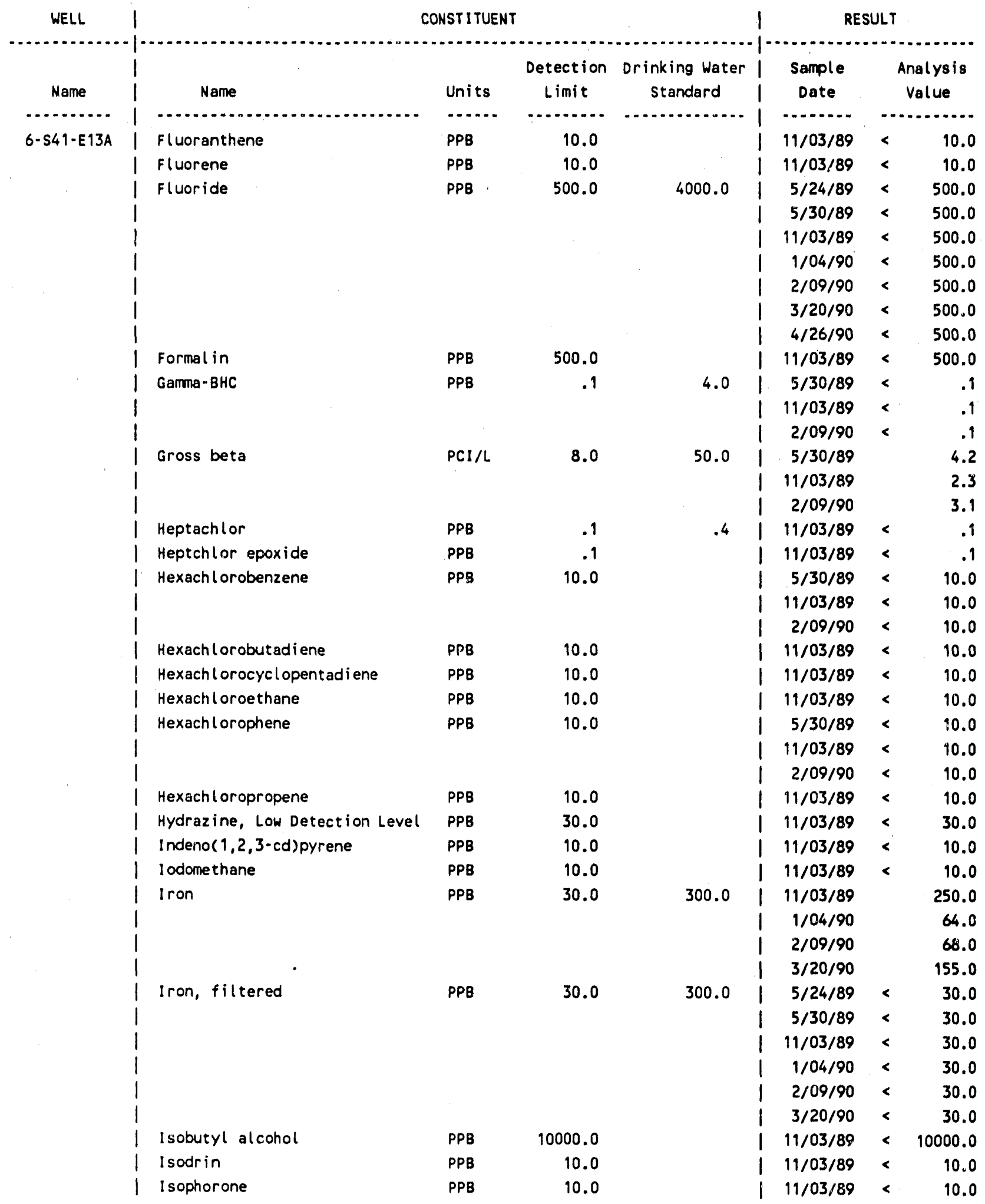




\section{WHC-MR. 0229}

$11 / 26 / 90$

Geosciences Group PARADOX Database

Well Result Report

1100 Area Groundwater Monitoring

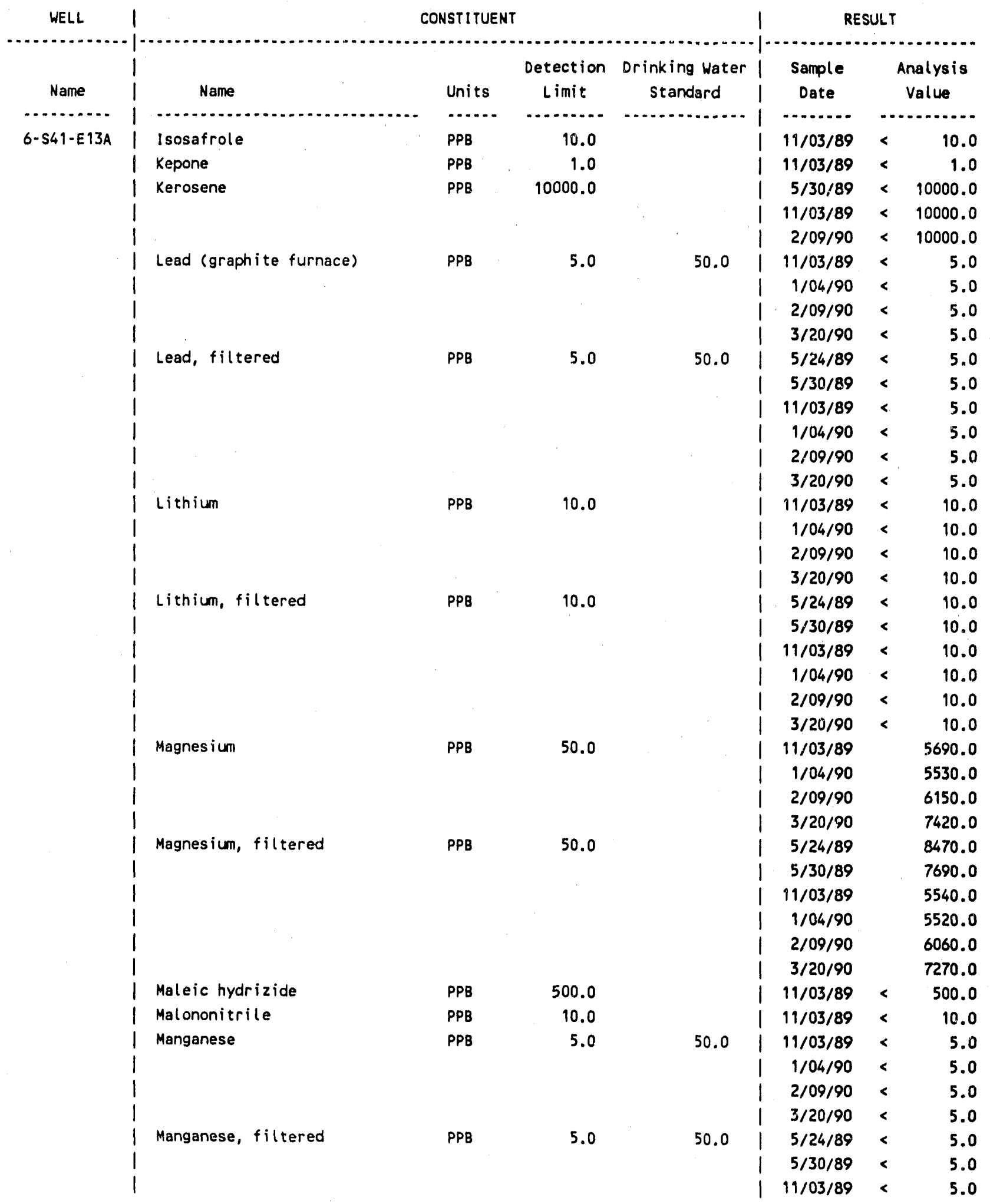




\section{WHC-MR. 0229}

$11 / 26 / 90$

Geosciences Group PARADOX Database

Well Result Report

Page 54

1100 Area Groundwater Monitoring

\begin{tabular}{|c|c|c|c|c|c|c|c|}
\hline WELL & \multicolumn{4}{|c|}{ CONSTI IUENT } & \multicolumn{3}{|c|}{ RESULT } \\
\hline Name & Name & Units & $\begin{array}{l}\text { Detection } \\
\text { Limit }\end{array}$ & $\begin{array}{c}\text { Drinking Water } \\
\text { Standard }\end{array}$ & $\begin{array}{c}\text { Sample } \\
\text { Date }\end{array}$ & & $\begin{array}{l}\text { ysis } \\
\text { We }\end{array}$ \\
\hline n. & | & -..... & - n........ & (n) & 1 $\ldots \ldots \ldots$ & $\cdots$ & ...... \\
\hline $6-541-E 13 A$ & I Manganese, filtered & PPB & 5.0 & 50.0 & $1 / 04 / 90$ & $<$ & 5.0 \\
\hline & 1 & & & & $2 / 09 / 90$ & $<$ & 5.0 \\
\hline & | & & & & $3 / 20 / 90$ & $<$ & 5.0 \\
\hline & I Melphalan & PPB & 10.0 & & | $11 / 03 / 89$ & $<$ & 10.0 \\
\hline & I Mercury & PPB & .1 & 2.0 & | $11 / 03 / 89$ & $<$ & .1 \\
\hline & 1 & & & & $1 \quad 2 / 09 / 90$ & $<$ & .1 \\
\hline & I Mercury, filtered & PPB & .1 & 2.0 & $5 / 30 / 89$ & $<$ & .1 \\
\hline & | & & & & I 11/03/89 & $<$ & .1 \\
\hline & | & & & & $1 \quad 2 / 09 / 90$ & $<$ & .1 \\
\hline & I Methacrylonitrile & PPB & 10.0 & & I 11/03/89 & $<$ & 10.0 \\
\hline & | Methanethiol & PPB & 10.0 & & | $11 / 03 / 89$ & $<$ & 10.0 \\
\hline & | Methapyrilene & PPB & 10.0 & & | $11 / 03 / 89$ & $<$ & 10.0 \\
\hline & I Metholonyl & PPB & 10.0 & & | 11/03/89 & $<$ & 10.0 \\
\hline & I Methoxychlor & PPB & 3.0 & 100.0 & $1 \quad 5 / 30 / 89$ & $<$ & 3.0 \\
\hline & | & & & & | 11/03/89 & $<$ & 3.0 \\
\hline & | & & & & $1 \quad 2 / 09 / 90$ & $<$ & 3.0 \\
\hline & I Methyl I sobutyl Ketone & PPB & 10.0 & & $5 / 24 / 89$ & $<$ & 10.0 \\
\hline & | & & & & $5 / 30 / 89$ & $<$ & 10.0 \\
\hline & I & & & & | 11/03/89 & $<$ & 10.0 \\
\hline & | & & & & $1 \quad 2 / 09 / 90$ & $<$ & 10.0 \\
\hline & I Methyl bromide & PPB & 10.0 & & | 11/03/89 & $<$ & 10.0 \\
\hline & Methyl chloride & PPB & 10.0 & & I $11 / 03 / 89$ & $<$ & 10.0 \\
\hline & Methyl ethyl ketone & PPB & 10.0 & & $15 / 24 / 89$ & $<$ & 10.0 \\
\hline & I & & & & $5 / 30 / 89$ & $<$ & 10.0 \\
\hline & | & & & & I $11 / 03 / 89$ & $<$ & 10.0 \\
\hline & | & & & & $12 / 09 / 90$ & $<$ & 10.0 \\
\hline & I Methyl methacrylate & PPB & 10.0 & & I $11 / 03 / 89$ & $<$ & 10.0 \\
\hline & I Methyl methanesul fonate & PPB & 10.0 & & I 11/03/89 & $<$ & 10.0 \\
\hline & Methyl parathion & PPB & 2.0 & & | 11/03/89 & $<$ & 2.0 \\
\hline & Methylene Chloride & PPB & 5.0 & & $15 / 24 / 89$ & $<$ & 5.0 \\
\hline & | & & & & $5 / 30 / 89$ & $<$ & 5.0 \\
\hline & | & & & & I 11/03/89 & $<$ & 5.0 \\
\hline & | & & & & $12 / 09 / 90$ & $<$ & 5.0 \\
\hline & Methyl thiouracil & PPB & 10.0 & & $111 / 03 / 89$ & \& & 10.0 \\
\hline & Molybdenum & PPB & 40.0 & & $111 / 03 / 89$ & $<$ & 40.0 \\
\hline & I & & & & I $1 / 04 / 90$ & $<$ & 40.0 \\
\hline & 1 & & & & $2 / 09 / 90$ & $<$ & 40.0 \\
\hline & | & & & & $3 / 20 / 90$ & $<$ & 40.0 \\
\hline & Molybdenum, filtered & PPB & 40.0 & & $5 / 24 / 89$ & $<$ & 40.0 \\
\hline & | & & & & $5 / 30 / 89$ & $<$ & 40.0 \\
\hline & I & & & & I 11/03/89 & $<$ & 40.0 \\
\hline & I & & & & $1 \quad 1 / 04 / 90$ & $<$ & 40.0 \\
\hline & 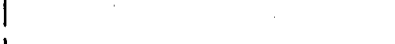 & & & & $2 / 09 / 90$ & $<$ & 40.0 \\
\hline & 1 & & & & $3 / 20 / 90$ & $<$ & 40.0 \\
\hline
\end{tabular}


WHC-MR. 0229

$11 / 26 / 90$

Geosoiences Group PARADOX Database

Well Result Report

Page 55

1100 Area Groundwater Monitoring

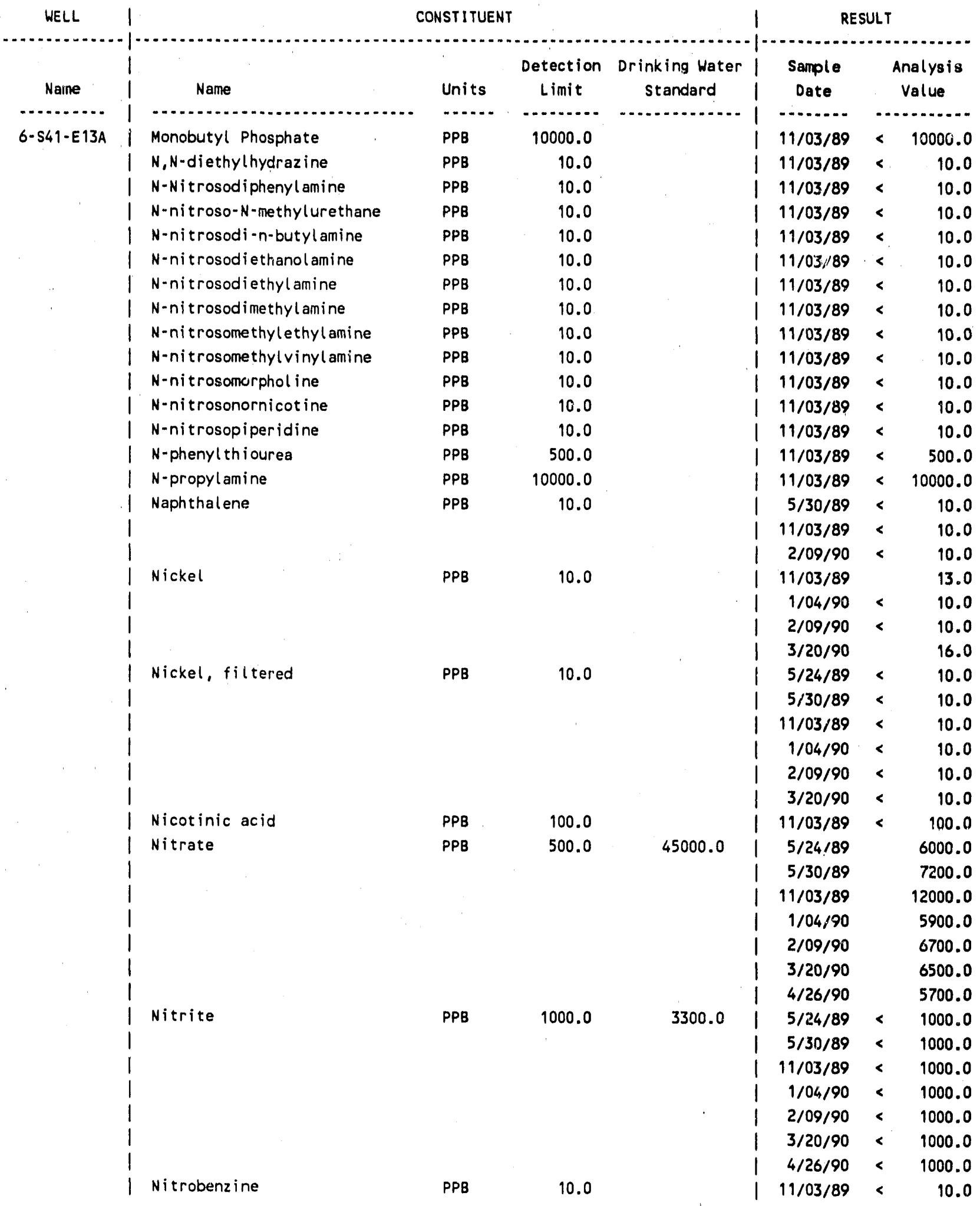


WHC-MR- 0229

Geosciences Group PARADOX Database

Well Result Report

1100 Area Groundwater Monitoring

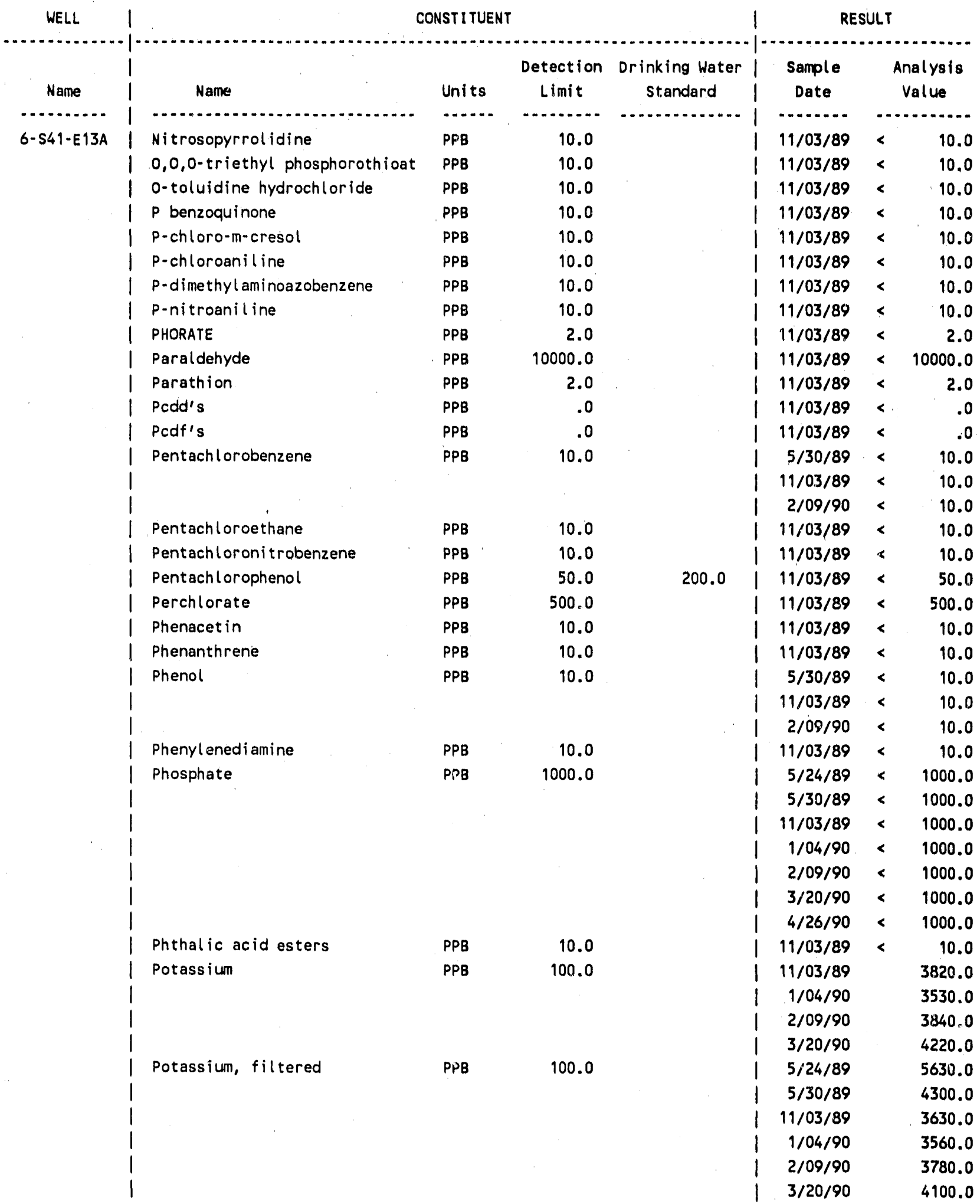


WHC-MR. 0229

Geosciences Group PARAOUX Oatabase

$11 / 26 / 90$

$$
\text { Well Result Report }
$$

1100 Area Groundwater Monitoring

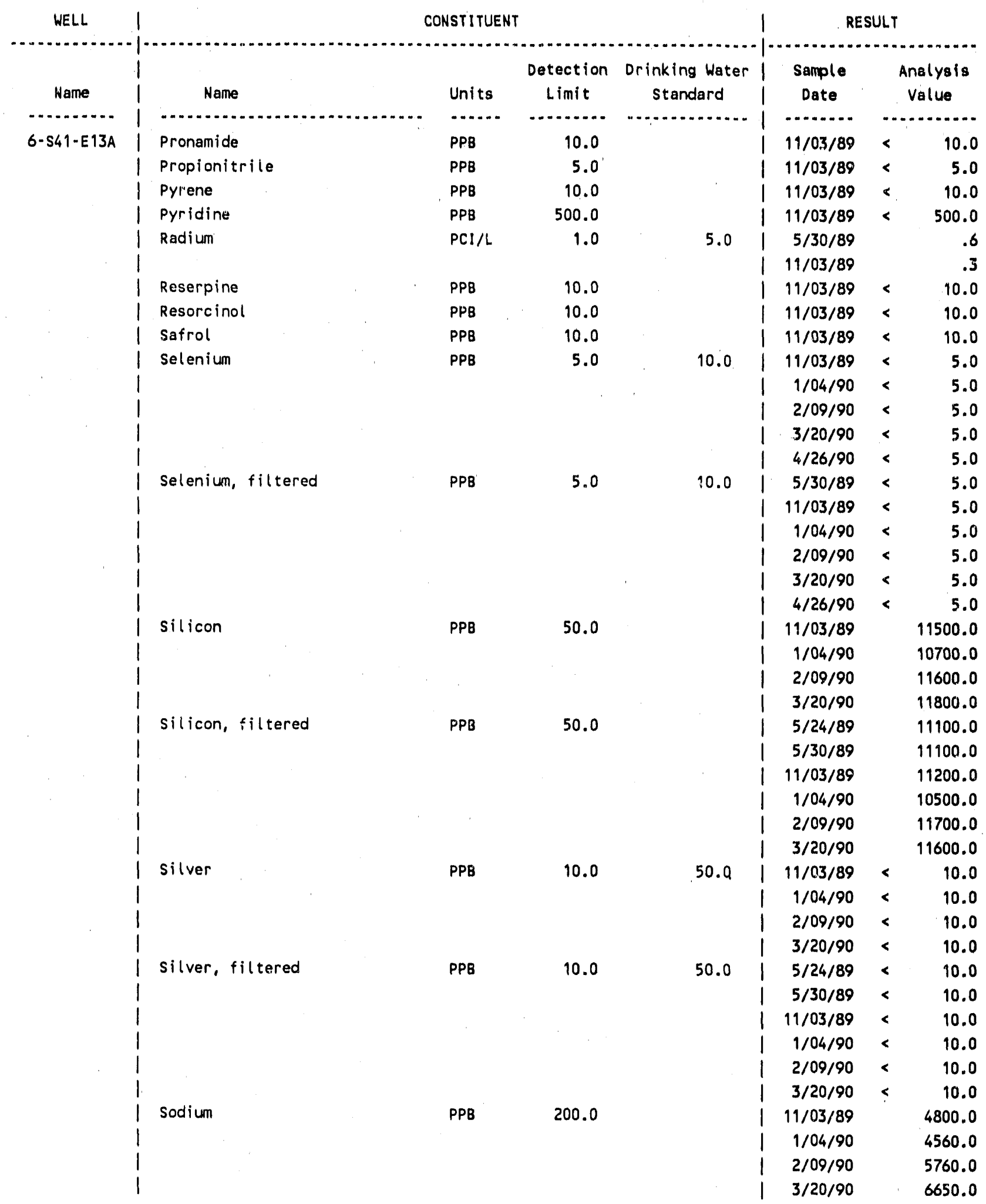


WHC-MR. 0229

$11 / 26 / 90$

Geosciences Group PARADOX Database

Well Result Report

1100 Area Groundwater Monitoring

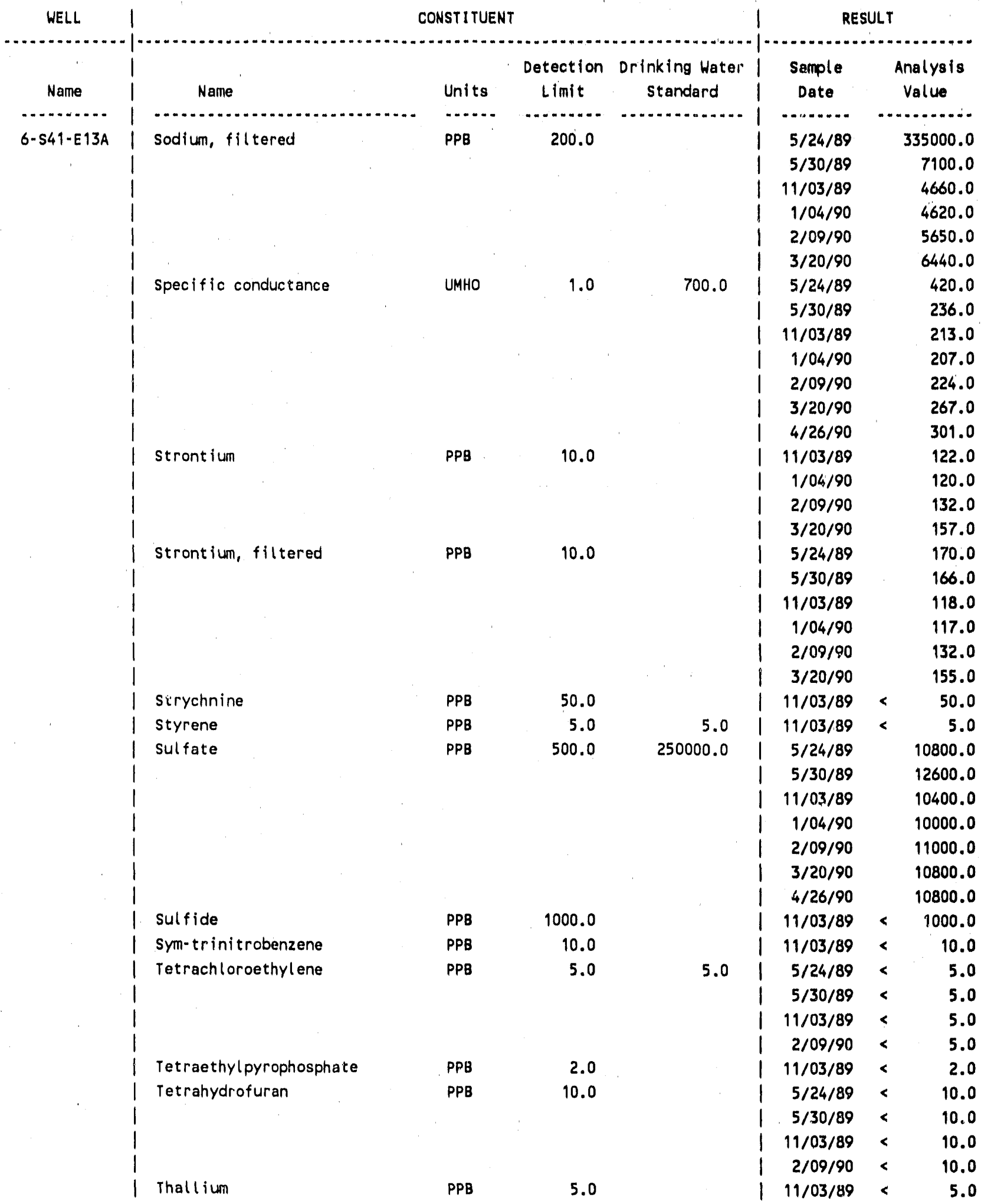


WHC.MR. 0229

$11 / 26 / 90$

Geosciences Group PARADOX Database

Well Result Report

Page 59

1100 Area Groundwater Monitoring

\begin{tabular}{|c|c|c|c|c|c|c|c|c|}
\hline WELL & 1 & & NST ITUEN & & & $R:$ & ULT & \\
\hline & 1. & & & Detection & Drinking Wuter & Sample & & nalysis \\
\hline Name & i & Name & Units & Limit & Standard & Date & & Value \\
\hline ….............. & i & - & ....... & $\ldots \ldots \ldots$ & $\ldots+\ldots$ & $\ldots \ldots$ & $\cdots$ & ........... \\
\hline $6.541-E 13 A$ & i & Thallium & PPB & 5.0 & & $2 / 09 / 90$ & $<$ & 5.0 \\
\hline & 1 & Thallium, filtered & PPB & 5.0 & & $5 / 30 / 89$ & $<$ & 5.0 \\
\hline & i & & & & & $11 / 03 / 89$ & $<$ & 5.0 \\
\hline & i & & & & & $2 / 09 / 90$ & $<$ & 5.0 \\
\hline & i & Thiofanox & PPB & 10.0 & & $11 / 03 / 89$ & $<$ & 10.0 \\
\hline & 1 & Thiourea & PPB & 200.0 & & $11 / 03 / 89$ & $<$ & 200.0 \\
\hline & i & Thiuram & PPB & 10.0 & & $11 / 03 / 89$ & $<$ & 10.0 \\
\hline & 1 & Tin & PPB & 30.0 & & $11 / 03 / 89$ & $<$ & 30.0 \\
\hline & i & & & & & $1 / 04 / 90$ & $<$ & 30.0 \\
\hline & i & & & & & $2 / 09 / 90$ & $<$ & 30.0 \\
\hline & i & & & & & $3 / 20 / 90$ & $<$ & 30.0 \\
\hline & i & Tin, filtered & PPB & 30.0 & & $5 / 24 / 89$ & $<$ & 30.0 \\
\hline & i & & & & & $5 / 30 / 89$ & $<$ & 30.0 \\
\hline & i & & & & & $11 / 03 / 89$ & $<$ & 30.0 \\
\hline & i & & & & & $1 / 04 / 90$ & $<$ & 30.0 \\
\hline & 1 & & & & & $2 / 09 / 90$ & $<$ & 30.0 \\
\hline & i & & & & & $3 / 20 / 90$ & $<$ & 30.0 \\
\hline & i & Titanium & PPB & 60.0 & & $11 / 03 / 89$ & $<$ & 60.0 \\
\hline & i & & & & & $1 / 04 / 90$ & $<$ & 60.0 \\
\hline & i & & & & & $2 / 09 / 90$ & $<$ & 60.0 \\
\hline & 1 & & & & & $3 / 20 / 90$ & $<$ & 60.0 \\
\hline & i & Titanium, filtered & PPB & 60.0 & & $5 / 24 / 89$ & $<$ & 60.0 \\
\hline & 1 & & & & & $5 / 30 / 89$ & 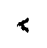 & 60.0 \\
\hline & 1 & & & & & $11 / 03 / 89$ & $<$ & 60.0 \\
\hline & i & & & & & $1 / 04 / 90$ & $<$ & 60.0 \\
\hline & i & & & & & $2 / 09 / 90$ & $<$ & 60.0 \\
\hline & 1 & & & & & $3 / 20 / 90$ & $<$ & 60.0 \\
\hline & 1 & Toluene & PPB & 5.0 & 2000.0 & $5 / 24 / 89$ & $<$ & 5.0 \\
\hline & 1 & & & & & $5 / 30 / 89$ & $<$ & 5.0 \\
\hline & 1 & & & & & $11 / 03 / 89$ & $<$ & 5.0 \\
\hline & i & & & & & $2 / 09 / 90$ & $<$ & 5.0 \\
\hline & I & Tolvenediamine & PPB & 10.0 & & $11 / 03 / 89$ & $<$ & 10.0 \\
\hline & i & Total Organic Halogen, Low Det & PPB & 10.0 & & $5 / 30 / 89$ & & 11.0 \\
\hline & 1 & & & & & $11 / 03 / 89$ & $<$ & 5.0 \\
\hline & 1 & & & & & $2 / 09 / 90$ & $<$ & 6.0 \\
\hline & 1 & Total carbon & PPB & 1000.0 & & $5 / 24 / 89$ & & 41400.0 \\
\hline & 1 & & & & & $5 / 30 / 89$ & & 29300.0 \\
\hline & 1 & & & & & $11 / 03 / 89$ & & 20900.0 \\
\hline & 1 & & & & & $2 / 09 / 90$ & & 23200.0 \\
\hline & 1 & Total organic carbon & PPB & 1000.0 & & $5 / 24 / 89$ & $<$ & 700.0 \\
\hline & 1 & & & & & $5 / 30 / 89$ & $<$ & 1400.0 \\
\hline & 1 & & & & & | $11 / 03 / 89$ & $<$ & 800.0 \\
\hline & 1 & & & & & | $2 / 09 / 90$ & $<$ & 700.0 \\
\hline & 1 & Toxaphene & PPB & 1.0 & 5.0 & $5 / 30 / 89$ & $<$ & 1.0 \\
\hline
\end{tabular}


WHC-MR. 0229

Geosciences Group PARADOX Database

$11 / 26 / 90$

Well Result Report

Page 60

1100 Area Groundwater Monitoring

\begin{tabular}{|c|c|c|c|c|c|c|c|}
\hline \multirow{2}{*}{$\begin{array}{l}\text { WELL } \\
\text { Name }\end{array}$} & \multicolumn{4}{|c|}{ CONSTI TUENT } & \multicolumn{3}{|c|}{ RESULT } \\
\hline & 1 & Units & $\begin{array}{l}\text { Detection } \\
\text { Limit }\end{array}$ & $\begin{array}{l}\text { Drinking Water } \\
\text { Standard }\end{array}$ & $\begin{array}{l}\text { Sample } \\
\text { Date }\end{array}$ & & $\begin{array}{l}\text { ysis } \\
\text { ue }\end{array}$ \\
\hline n.......... & | & $\cdots+\cdots$ & (n......... & (n) & 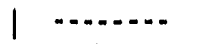 & & ...... \\
\hline $6.541-E 13 A$ & I Toxaphene & PPB & 1.0 & 5.0 & $11 / 03 / 89$ & $<$ & 1.0 \\
\hline & 1 & & & & $2 / 09 / 90$ & $<$ & 1.0 \\
\hline & I Trans-1,2-dichloroethene & PPB & 5.0 & 70.0 & $5 / 24 / 89$ & $<$ & 5.0 \\
\hline & $i$ & & & & $5 / 30 / 89$ & $<$ & 5.0 \\
\hline & 1 & & & & $11 / 03 / 89$ & $<$ & 5.0 \\
\hline & 1 & & & & i $2 / 09 / 90$ & $<$ & 5.0 \\
\hline & | Tributy!phosphoric Acid. & PPB & 10.0 & & $5 / 30 / 89$ & $<$ & 10.0 \\
\hline & 1 & & & & | 11/03/89 & $<$ & 10.0 \\
\hline & 1 & & & & $2 / 09 / 90$ & - & 10.0 \\
\hline & | Trichloroethylene & PPB & 5.0 & 5.0 & $5 / 24 / 89$ & $<$ & 5.0 \\
\hline & 1 & & & & $5 / 30 / 89$ & $<$ & 5.0 \\
\hline & I & & & & | 11/03/89 & $<$ & 5.0 \\
\hline & 1 & & & & 1 $2 / 09 / 90$ & $<$ & 5.0 \\
\hline & | Trichlorome thanethiol & PPB & 10.0 & & $11 / 03 / 89$ & $<$ & 10.0 \\
\hline & I Trichloromonof luorome thane & PPB & 10.0 & & 11/03/89 & $<$ & 10.0 \\
\hline & I Tris(2,3-dibromopropyl) phosph & PPB & 10.0 & & $11 / 03 / 89$ & $<$ & 10.0 \\
\hline & I Tritium & $\mathrm{PCl} / \mathrm{L}$ & 500.0 & 20000.0 & $5 / 30 / 89$ & $<$ & 58.4 \\
\hline & 1 & & & & $111 / 03 / 89$ & $<$ & 83.6 \\
\hline & I Turbidity & NTU & .1 & 5.0 & | 11/03/89 & & 3.6 \\
\hline & 1 & & & & $2 / 09 / 90$ & & 1.1 \\
\hline & I Vanadium & PPB & 5.0 & & | 11/03/89 & $<$ & 5.0 \\
\hline & 1 & & & & $1 / 04 / 90$ & & 7.0 \\
\hline & 1 & & & & $2 / 09 / 90$ & & 7.0 \\
\hline & 1 & & & & $3 / 20 / 90$ & & 8.0 \\
\hline & I Vanadium, filtered & PPB & 5.0 & & $5 / 24 / 89$ & & 10.0 \\
\hline & 1 & & & & $5 / 30 / 89$ & & 9.0 \\
\hline & 1 & & & & | 11/03/89 & $<$ & 5.0 \\
\hline & 1 & & & & $1 / 04 / 90$ & & 6.0 \\
\hline & 1 & & & & $2 / 09 / 90$ & & 6.0 \\
\hline & 1 & & & & $3 / 20 / 90$ & & 7.0 \\
\hline & I Vinyl Acetate & PPB & 5.0 & & | 11/03/89 & $<$ & 5.0 \\
\hline & I Vinyl chloride & PPB & 10.0 & 2.0 & $1 \quad 5 / 24 / 89$ & $<$ & 10.0 \\
\hline & 1 & & & & $5 / 30 / 89$ & $<$ & 10.0 \\
\hline & 1 & & & & | 11/03/89 & $<$ & 10.0 \\
\hline & 1 & & & & $1 \quad 2 / 09 / 90$ & $<$ & 10.0 \\
\hline & I Warfarin & PPB & 10.0 & & | 11/03/89 & $<$ & 10.0 \\
\hline & I xylene-m & PPB & 5.0 & 10000.0 & | $5 / 24 / 89$ & $<$ & 5.0 \\
\hline & 1 & & & & $5 / 30 / 89$ & $<$ & 5.0 \\
\hline & 1 & & & & | 11/03/89 & $<$ & 5.0 \\
\hline & 1 & & & & $2 / 09 / 90$ & $<$ & 5.0 \\
\hline & $\mid x y l$ ene-0,p & PPB & 5.0 & 10000.0 & $5 / 24 / 89$ & $<$ & 5.0 \\
\hline & 1 & & & & $5 / 30 / 89$ & $<$ & 5.0 \\
\hline & 1 & & & & | 1 11/03/89 & $<$ & 5.0 \\
\hline & 1 & & & & | $2 / 09 / 90$ & $<$ & 5.0 \\
\hline
\end{tabular}


WHC-MR- 0229

$11 / 26 / 90$

Geosciences Group PARADOX Database

Well Result Report

Page 61

1100 Area Groundwater Monitoring

\begin{tabular}{|c|c|c|c|c|c|c|c|}
\hline \multirow{2}{*}{$\begin{array}{c}\text { WELL } \\
\text { Name }\end{array}$} & 1 & \multicolumn{3}{|c|}{ CONSTITUENT } & \multicolumn{3}{|c|}{ RESULT } \\
\hline & 1 & Units & $\begin{array}{l}\text { Detection } \\
\text { Limit }\end{array}$ & $\begin{array}{l}\text { Drinking Water } \\
\text { standard }\end{array}$ & $\begin{array}{l}\text { Sample } \\
\text { Date }\end{array}$ & & $\begin{array}{l}\text { Analysis } \\
\text { Value }\end{array}$ \\
\hline …......... & | & ….... & n......... & 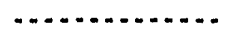 & | $\cdots . . . .$. & & ............ \\
\hline $6-541-E 13 A$ & I zinc & PPB & 5.0 & 5000.0 & 1. $11 / 03 / 89$ & & 5.0 \\
\hline & $i$ & & & & $1 / 04 / 90$ & $<$ & 5.0 \\
\hline & 1 & & & & $2 / 09 / 90$ & $<$ & 5.0 \\
\hline & 1 & & & & $3 / 20 / 90$ & $<$ & 5.0 \\
\hline & I zinc, filtered & PPB & 5.0 & 5000.0 & $5 / 24 / 89$ & $<$ & 5.0 \\
\hline & 1 & & & & $5 / 30 / 89$ & $<$ & 5.0 \\
\hline & 1 & & & & I 11/03/89 & & 9.0 \\
\hline & 1 & & & & $1 \quad 1 / 04 / 90$ & & 5.0 \\
\hline & 1 & & & & $2 / 09 / 90$ & $<$ & 5.0 \\
\hline & 1 & & & & $3 / 20 / 90$ & $<$ & 5.0 \\
\hline & I zirconium & PPB & 50.0 & & | 11/03/89 & $<$ & 50.0 \\
\hline & 1 & & & & $1 / 04 / 90$ & $<$ & 50.0 \\
\hline & 1 & & & & $2 / 09 / 90$ & < & 50.0 \\
\hline & 1 & & & & $3 / 20 / 90$ & $<$ & 50.0 \\
\hline & I zirconium, filtered & PPB & 50.0 & & $5 / 24 / 89$ & $<$ & 50.0 \\
\hline & 1 & & & & $5 / 30 / 89$ & $<$ & 50.0 \\
\hline & 1 & & & & | 11/03/89 & $<$ & 50.0 \\
\hline & 1 & & & & $1 \quad 1 / 04 / 90$ & $<$ & 50.0 \\
\hline & 1 & & & & $2 / 09 / 90$ & $<$ & 50.0 \\
\hline & i & & & & $3 / 20 / 90$ & $<$ & 50.0 \\
\hline & I dibromochloromethane & PPB & 5.0 & 100.0 & I 11/03/89 & $<$ & 5.0 \\
\hline & I m-Nitroaniline & PPB & 10.0 & & $11 / 03 / 89$ & $<$ & 10.0 \\
\hline & I o-Nitroaniline & PPB & 10.0 & & $11 / 03 / 89$ & $<$ & 10.0 \\
\hline & I o-Nitrophenol & PPB & 10.0 & & | 11/03/89 & $<$ & 10.0 \\
\hline & | p-Dichlorobenzene & PPB & 5.0 & 750.0 & | 5/24/89 & $<$ & 5.0 \\
\hline & 1 & & & & $5 / 30 / 89$ & $<$ & 5.0 \\
\hline & 1 & & & & | 11/03/89 & $<$ & 5.0 \\
\hline & 1 & & & & | $2 / 09 / 90$ & $<$ & 5.0 \\
\hline & 1 & & 10.0 & 750.0 & $5 / 30 / 89$ & $<$ & 10.0 \\
\hline & 1 & & & & | 11/03/89 & $<$ & 10.0 \\
\hline & 1 & & & & $2 / 09 / 90$ & 3 & 10.0 \\
\hline & I p-Nitrophenol & PPB & 10.0 & & | 11/03/89 & $<$ & 10.0 \\
\hline & I pH, Field Measurement & & .1 & 8.5 & $5 / 24 / 89$ & & 7.8 \\
\hline & 1 & & & & $5 / 30 / 89$ & & 7.6 \\
\hline & 1 & & & & $111 / 03 / 89$ & & 8.1 \\
\hline & 1 & & & & $1 / 04 / 90$ & & 8.1 \\
\hline & 1 & & & & $2 / 09 / 90$ & & 7.9 \\
\hline & 1 & & & & $3 / 20 / 90$ & & 7.9 \\
\hline & 1 & & & & $4 / 26 / 90$ & & 7.8 \\
\hline & I pH, Laboratory Measurement & & .0 & 8.5 & $5 / 24,89$ & & 7.9 \\
\hline & 1 & & & & $5 / 30 / 89$ & & 7.9 \\
\hline & 1 & & & & $111 / 03 / 89$ & & 8.0 \\
\hline & 1 & & & & $12 / 09 / 90$ & & 7.8 \\
\hline & 1 & & & & 1 & & \\
\hline
\end{tabular}




\section{WHC-MK- 0229}

Hell Result Report

1100 Area Groundwater Monitoring

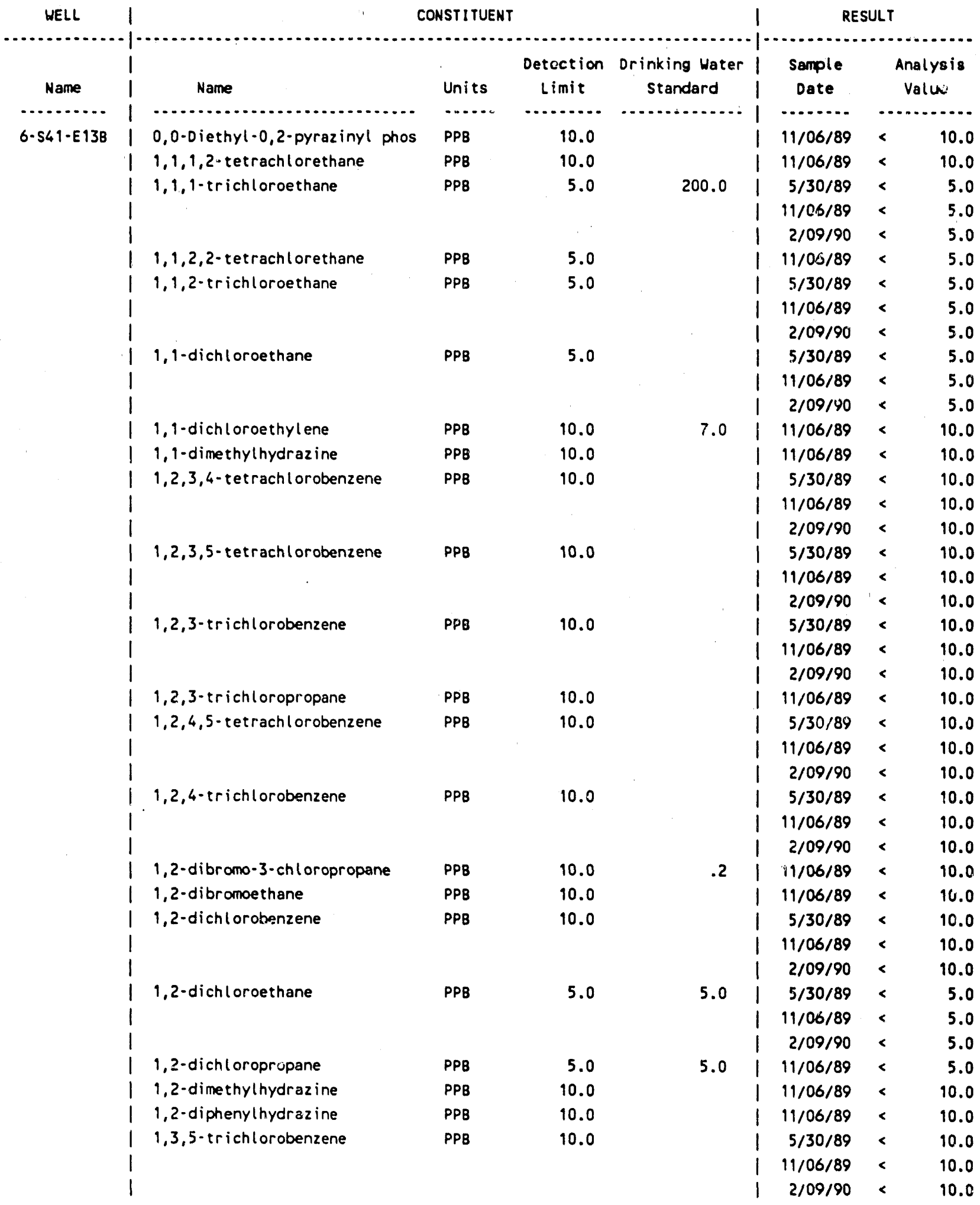




\section{WHC-MR. 0229}

Hell Result Report

1100 Area Groundwater Monitoring

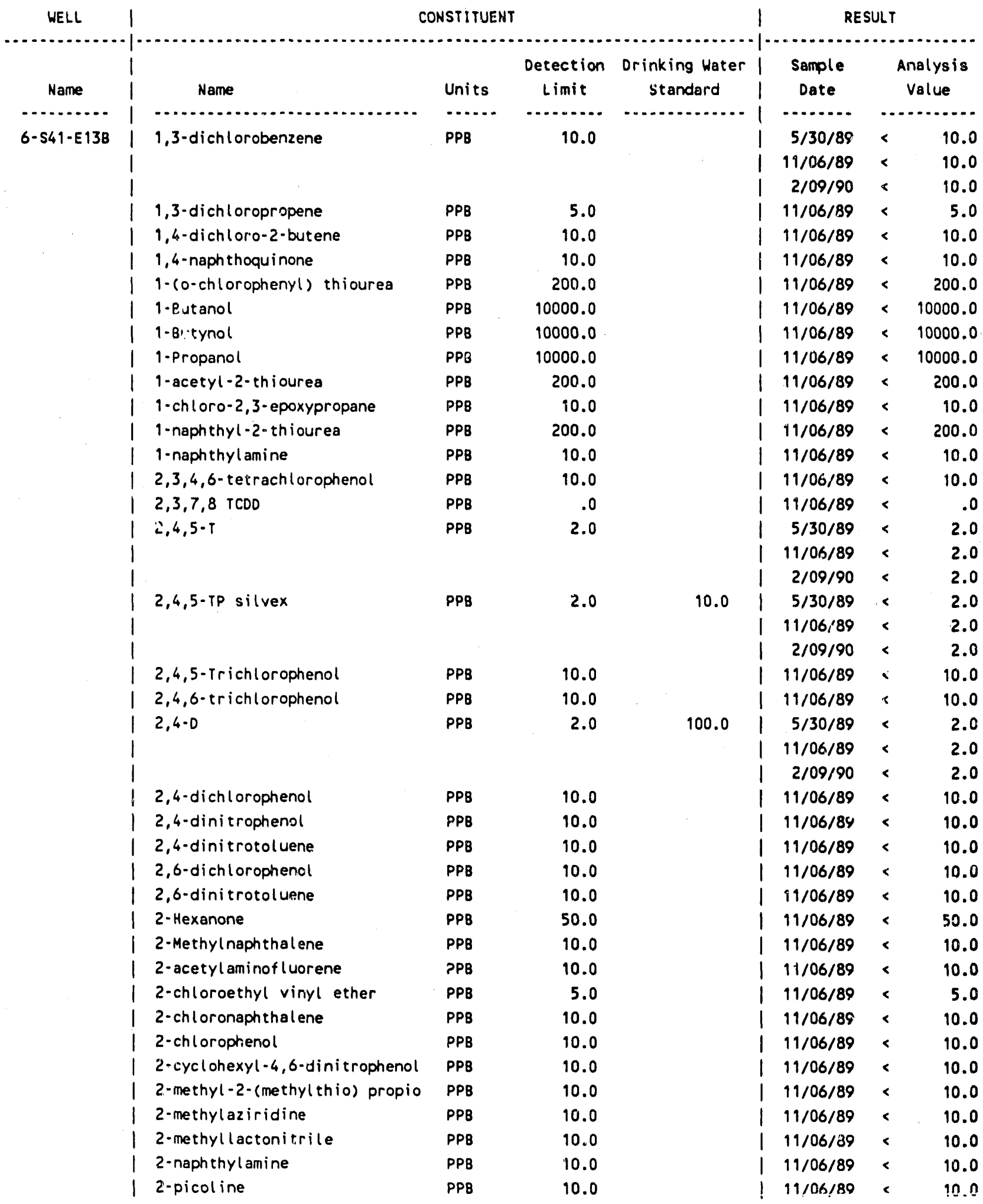




\section{WHC-MR- 0229}

$11 / 26 / 90$

Geosciences Group PARADDX Database

Well Result Report

1100 Area Groundwater Monitoring

\begin{tabular}{|c|c|c|c|c|c|c|c|c|}
\hline \multirow{2}{*}{$\begin{array}{c}\text { WELL } \\
\ldots \\
\text { Name }\end{array}$} & 1 & \multicolumn{3}{|c|}{ ONSTIITUENT } & 1 & \multicolumn{3}{|c|}{ RESULT } \\
\hline & Name & Units & $\begin{array}{l}\text { Detection } \\
\text { Limit }\end{array}$ & $\begin{array}{c}\text { Drinking Water } \\
\text { Standard }\end{array}$ & 1 & $\begin{array}{c}\text { Sample } \\
\text { Date }\end{array}$ & & $\begin{array}{l}\text { Analysis } \\
\text { Value }\end{array}$ \\
\hline ............ & 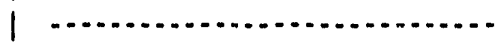 & ...... & .......... & tenter & 1 & ......... & & ........... \\
\hline $6-S 41-E 13 B$ & | 2-propyn-1-ol & PPB & 10000.0 & & i & $11 / 06 / 89$ & $<$ & 10000.0 \\
\hline & 2-sec-butyl-4,6-dinitrophenol & PPB & 10.0 & & 1 & $11 / 06 / 89$ & $<$ & 10.0 \\
\hline & | 3,3'-Dichlorobenzidine & PPB & 10.0 & & 1 & $11 / 06 / 89$ & $<$ & 10.0 \\
\hline & | 3,31-dimethoxybenzidine & PPB & 10.0 & & i & $11 / 06 / 89$ & $<$ & 10.0 \\
\hline & 1 3,31-dimethylbenzidine & PPB & 10.0 & & i & $11 / 06 / 89$ & $<$ & 10.0 \\
\hline & 3-chloropropionitrile & PPB & 10000.0 & & i & $11 / 06 / 89$ & $<$ & 10000.0 \\
\hline & | 3-methylcholanthrene & PPB & 10.0 & & 1 & $11 / 06 / 89$ & $<$ & 10.0 \\
\hline & i 4,4'-methylenebis (2-chloroanil & PPB & 10.0 & & i & $11 / 06 / 89$ & $<$ & 10.0 \\
\hline & 4,6-dinitro-0-cresol and salts & PPB & 10.0 & & i & $11 / 06 / 89$ & $<$ & 10.0 \\
\hline & | 4-Nitroquinoline 1-oxide & PPB & 10.0 & & i & $11 / 06 / 89$ & $<$ & 10.0 \\
\hline & | 4-aminobyphenyl & PPB & 10.0 & & 1 & $11 / 06 / 89$ & $<$ & 10.0 \\
\hline & I 4-bromophenyl phenyl ether & PPB & 10.0 & & $i$ & $11 / 06 / 89$ & $<$ & 10.0 \\
\hline & 1 5-(aminomethyl)-3-isoxazolol & PPB & 10.0 & & i & $11 / 06 / 89$ & $<$ & 10.0 \\
\hline & | 5-nitro-o-toluidine & PPB & 10.0 & & i & $11 / 06 / 89$ & $<$ & 10.0 \\
\hline & 1 7,12-dimethylbenz [a] anthracene & PPB & 10.0 & & 1 & $11 / 06 / 89$ & $<$ & 10.0 \\
\hline & | $7 H$-dibenzo $[c, g]$ carbazole & PPB & 10.0 & & 1 & $11 / 06 / 89$ & $<$ & 10.0 \\
\hline & I Acenaphthalene & PPB & 10.0 & & i & $11 / 06 / 89$ & $<$ & 10.0 \\
\hline & I Acenaphthene & PPB & 10.0 & & i & $11 / 06 / 89$ & $<$ & 10.0 \\
\hline & I Acetone - by ABN & PPB & 10.0 & & I & $11 / 06 / 89$ & $<$ & 10.0 \\
\hline & I Acetone by VOA & PPB & 10.0 & & 1 & $5 / 30 / 89$ & $<$ & 10.0 \\
\hline & 1 & & & & i & $11 / 06 / 89$ & $<$ & 10.0 \\
\hline & 1 & & & & 1 & $2 / 09 / 90$ & $<$ & 10.0 \\
\hline & I Acetonitrile & PPB & 10.0 & & i & $11 / 06 / 89$ & $<$ & 10.0 \\
\hline & Acetophenone & PPB & 10.0 & & i & $11 / 06 / 89$ & $<$ & 10.0 \\
\hline & I Acrolein & PPB & 10.0 & & 1 & $11 / 06 / 89$ & $<$ & 10.0 \\
\hline & I Acrylamide & PPB & 10000.0 & & i & $11 / 06 / 89$ & $<$ & 10000.0 \\
\hline & I Acrylonitrile & PPB & 10.0 & & i & $11 / 06 / 89$ & $<$ & 10.0 \\
\hline & I Aldrin & PPB & .1 & & i & $11 / 06 / 89$ & $<$ & .1 \\
\hline & I Alkalinity & & 20000.0 & & 1 & $5 / 30 / 89$ & & 217000.0 \\
\hline & 1 & & & & i & $11 / 06 / 89$ & & 206000.0 \\
\hline & I Allyl Chloride & PPB & 100.0 & & 1 & $11 / 06 / 89$ & $<$ & 100.0 \\
\hline & I Allyl alcohol & PPB & 10000.0 & & i & $11 / 06 / 89$ & $<$ & 10000.0 \\
\hline & I Alpha, High Detection Level & $\mathrm{PCI} / \mathrm{L}$ & 4.0 & 15.0 & i & $5 / 30 / 89$ & & 5.7 \\
\hline & 1 & & & & 1 & $11 / 06 / 89$ & & 3.2 \\
\hline & 1 & & & & 1 & $2 / 09 / 90$ & & 3.3 \\
\hline & I Alpha, alpha-dimethyl phenethyla & PPB & 10.0 & & i & $11 / 06 / 89$ & $<$ & 10.0 \\
\hline & I Alpha-BHC & PPB & .1 & 4.0 & i & $5 / 30 / 89$ & $<$ &. \\
\hline & 1 & & & & 1 & $11 / 76 / 89$ & $<$ & .1 \\
\hline & 1 & & & & 1 & $2 / 09 / 90$ & $<$ & .1 \\
\hline & Aluminum & PPB & 150.0 & 50.0 & i & $11 / 06 / 89$ & $<$ & 150.0 \\
\hline & 1 & & & & i & $1 / 04 / 90$ & $<$ & 150.0 \\
\hline & 1 & & & & I & $2 / 09 / 90$ & $<$ & 150.0 \\
\hline & 1 & & & & i & $3 / 20 / 90$ & $<$ & 150.0 \\
\hline & I Aluminum, filtered & PPB & 150.0 & 50.0 & i & $5 / 30 / 89$ & $<$ & 150.0 \\
\hline
\end{tabular}




\section{WHC-MR. 0229}

Geosciences Group PARADOX Database

Well Result Report

1100 Area Groundwater Monitoring

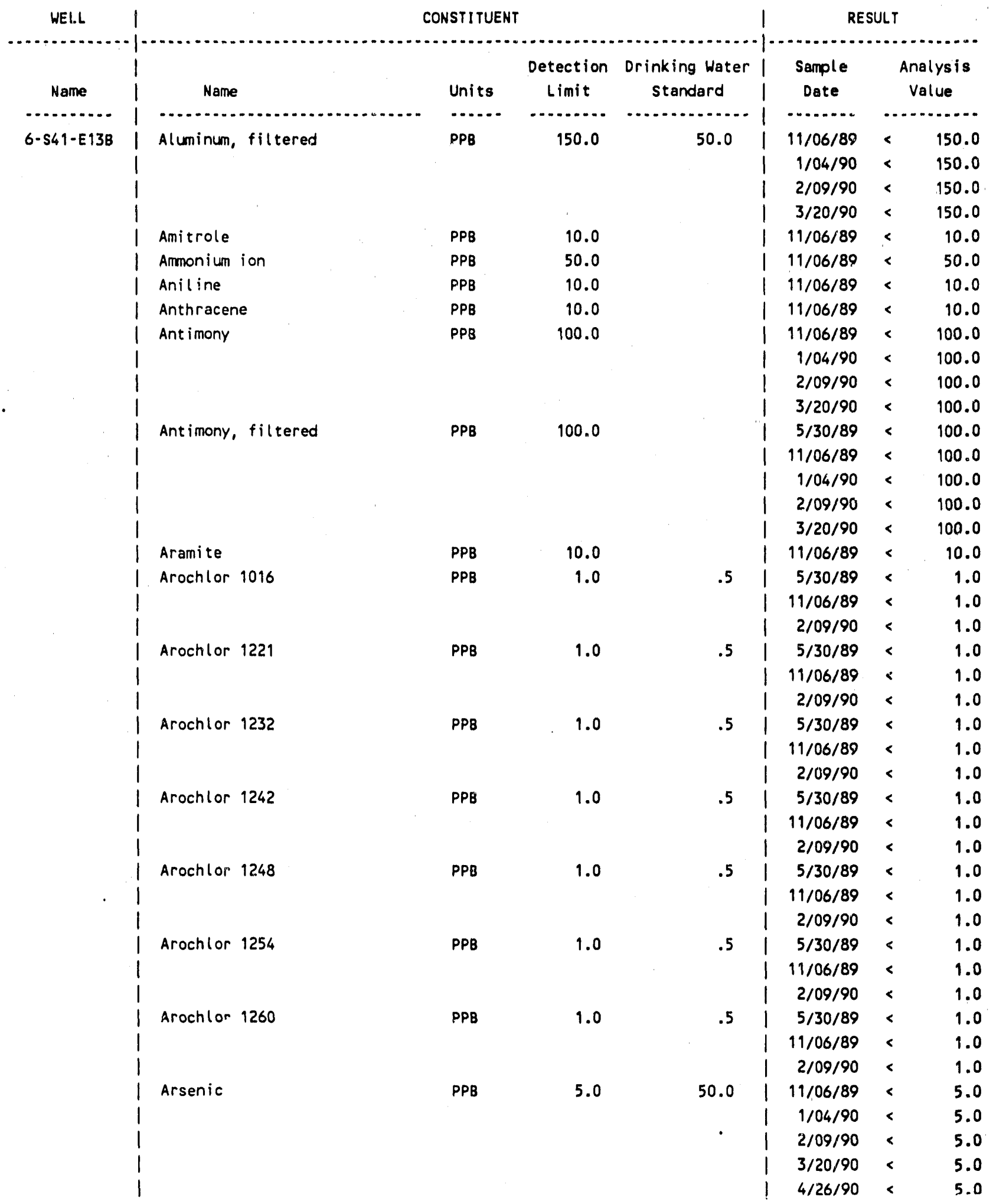




\section{WHC-MR- 0229}

$11 / 26 / 90$

Geosciences Group PARADOX Database

Well Result Report

Page 66

1100 Area Groundwater Monitoring

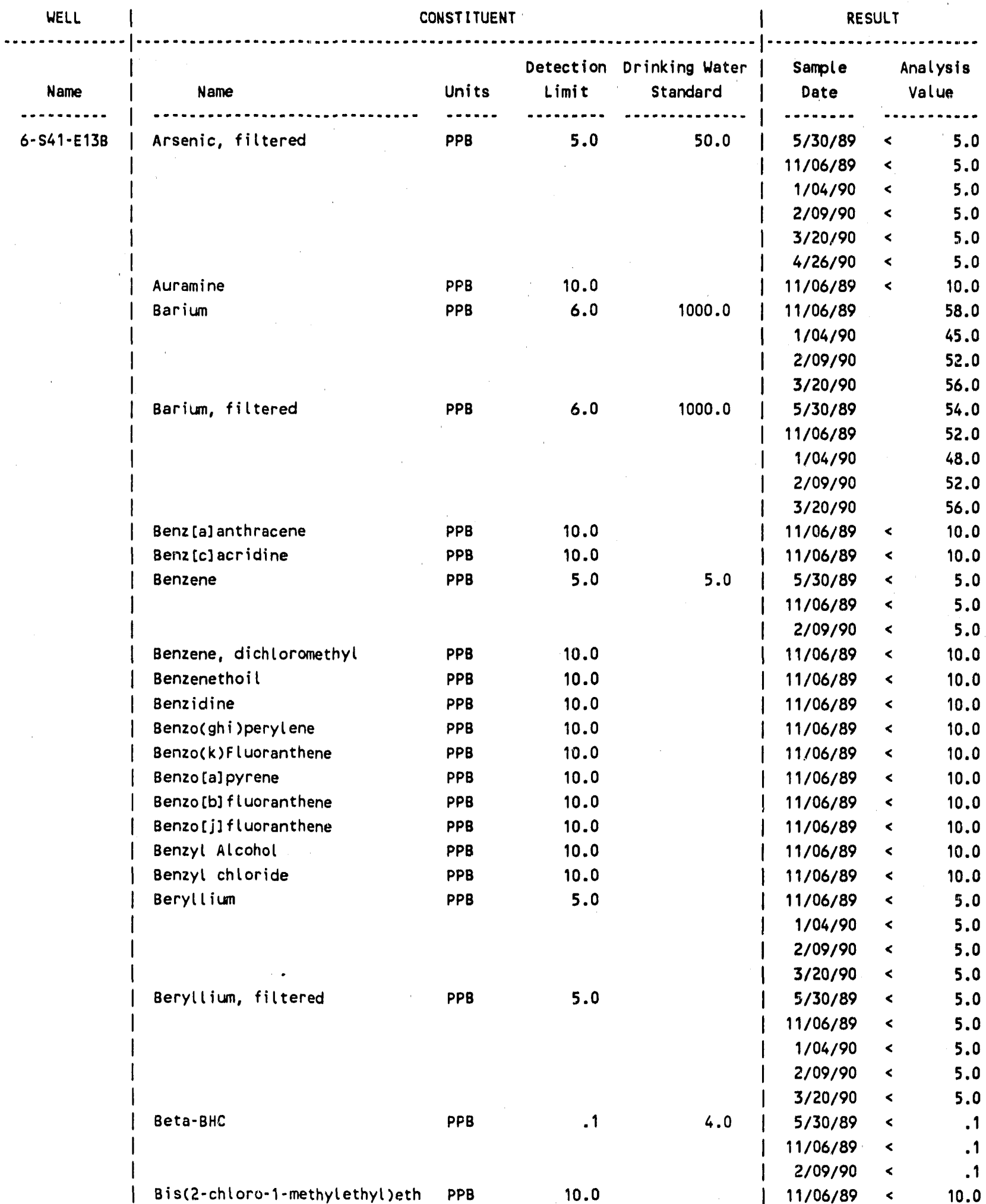


WHC-MR. 0229

$11 / 26 / 90$

Geosciences Group PARADOX Database

Page 67

Well Result Repore

1100 Area Groundwater Monitoring

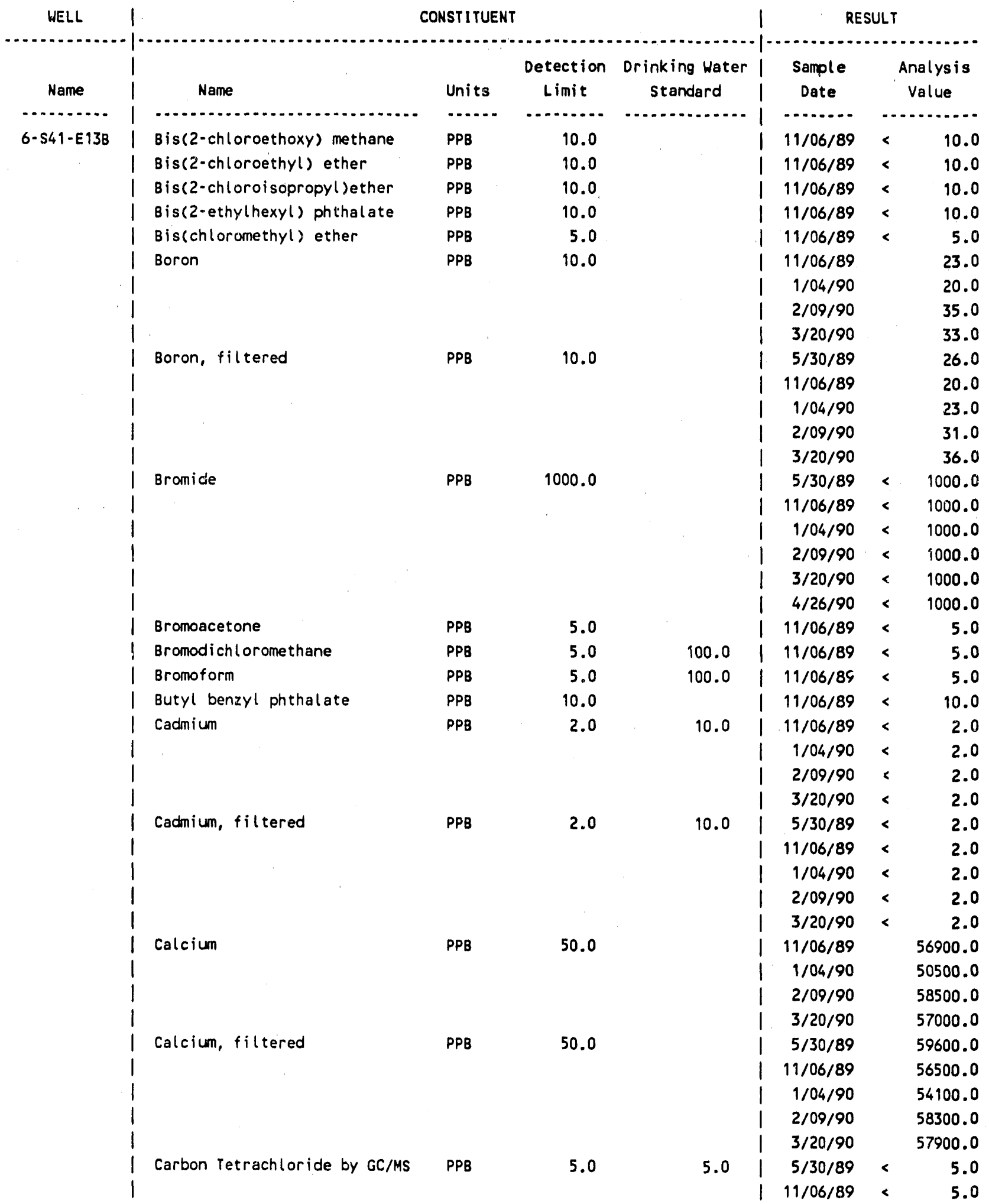




\section{WHC-MR. 0229}

$11 / 26 / 90$

Geosciences Group PARADOX Database

Well Result Report

1100 Area Groundwater Monitoring

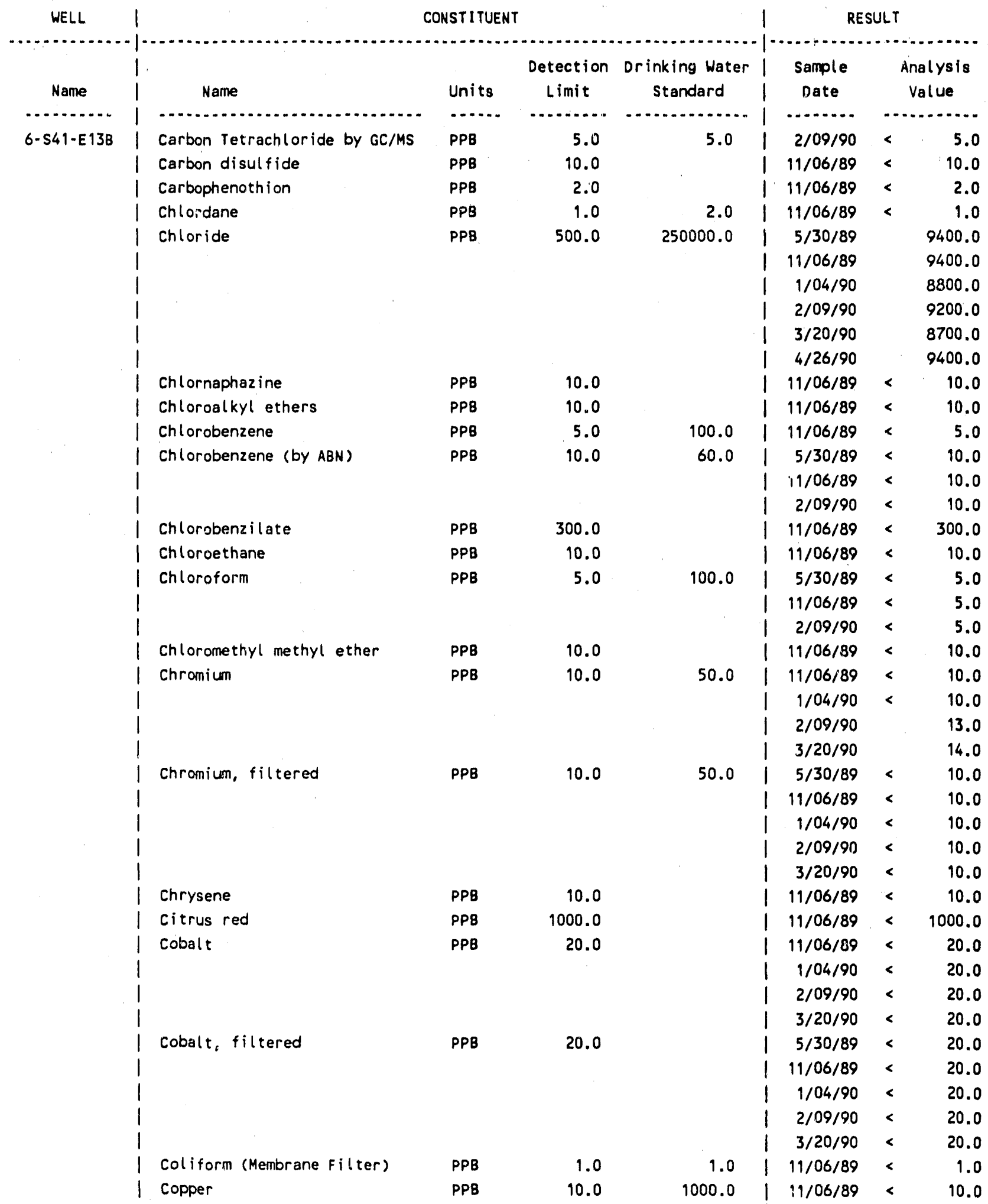




\section{WHC-MR. 0229}

Well Result Report

1100 Area Groundwater Monitoring

\begin{tabular}{|c|c|c|c|c|c|c|c|c|c|}
\hline WELL & 1 & \multicolumn{5}{|c|}{ CONSTITUENT } & \multicolumn{3}{|c|}{ RESULT } \\
\hline & I & & & Detection & Drinking Water & 1 & Sample & & inalysis \\
\hline Name & 1 & Name & Units & Limit & Standard & 1 & Date & & Value \\
\hline$\cdots \ldots \ldots$ & 1 & ( & $\cdots+$ & n.......... & 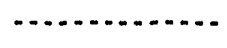 & & ............ & $\cdots$ & ........... \\
\hline $6-541-E 13 B$ & 1 & Copper & PPB & 10.0 & 1000.0 & 1 & $1 / 04 / 90$ & $<$ & 10.0 \\
\hline & 1 & & & & & 1 & $2 / 09 / 90$ & $<$ & 10.0 \\
\hline & 1 & & & & & i & $3 / 20 / 90$ & $<$ & 10.0 \\
\hline & 1 & Copper, filtered & PPB & 10.0 & 1000.0 & I & $5 / 30 / 89$ & $<$ & 10.0 \\
\hline & 1 & & & & & i & $11 / 06 / 89$ & $<$ & 10.0 \\
\hline & 1 & & & & & I & $1 / 04 / 90$ & $<$ & 10.0 \\
\hline & 1 & & & & & i & $2 / 09 / 90$ & $<$ & 10.0 \\
\hline & 1 & & & & & I & $3 / 20 / 90$ & $<$ & 10.0 \\
\hline & 1 & Cresols & PPB & 10.0 & & i & $11 / 06 / 89$ & $<$ & 10.0 \\
\hline & 1 & Crotonaldehyde & PPB & 10.0 & & 1 & $11 / 06 / 89$ & $<$ & 10.0 \\
\hline & 1 & Cyanide & PPB & 10.0 & & i & $11 / 06 / 89$ & $<$ & 10.0 \\
\hline & 1 & DDD & PPB & .9 & & 1 & $11 / 06 / 89$ & $<$ & .1 \\
\hline & i & DDE & PPB & .1 & & i & $11 / 06 / 89$ & $<$ & .1 \\
\hline & 1 & DOT & PPB & .1 & & 1 & $11 / 06 / 89$ & $<$ & .1 \\
\hline & 1 & Delta-BHC & PPB & .1 & 4.0 & i & $5 / 30 / 89$ & $<$ & .1 \\
\hline & 1 & & & & & 1 & $11 / 06 / 89$ & $<$ & .1 \\
\hline & I & & & & & I & $2 / 09 / 90$ & $<$ & .1 \\
\hline & 1 & Di-n-butyl phthalate & PPB & 10.0 & & i & $11 / 06 / 89$ & $<$ & 10.0 \\
\hline & 1 & Di-n-octyl phehalate & PPB & 10.0 & & 1 & $11 / 06 / 89$ & $<$ & 10.0 \\
\hline & 1 & Di-n-propylnitrosamine & PPB & 10.0 & & 1 & $11 / 06 / 89$ & $<$ & 10.0 \\
\hline & 1 & Diallate & PPB & 10.0 & & 1 & $11 / 06 / 89$ & $<$ & 10.0 \\
\hline & 1 & Dibenz $[a, h]$ acridine & PPB & 10.0 & & 1 & $11 / 06 / 89$ & $<$ & 10.0 \\
\hline & 1 & Dibenz $[a, h]$ anthracene & PPB & 10.0 & & 1 & $11 / 06 / 89$ & $<$ & 10.0 \\
\hline & 1 & Dibenz $[a, j]$ acridine & PPB & 10.0 & & 1 & $11 / 06 / 89$ & $<$ & 10.0 \\
\hline & 1 & Dibenzo[a, e]pyrene & PPB & 10.0 & & 1 & $11 / 06 / 89$ & $<$ & 10.0 \\
\hline & 1 & Dibenzo $[a, h]$ pyrene & PPB & 10.0 & & 1 & $11 / 06 / 89$ & $<$ & 10.0 \\
\hline & 1 & Dibenzo[a, i]pyrene & PPB & 10.0 & & 1 & $11 / 06 / 89$ & $<$ & 10.0 \\
\hline & 1 & Dibenzofuran & PPB & 10.0 & & 1 & $11 / 06 / 89$ & $<$ & 10.0 \\
\hline & 1 & Dibromome thane & PPB & 10.0 & & 1 & $11 / 06 / 89$ & $<$ & 10.0 \\
\hline & 1 & Dibutyl Phosphate & PPB & 10000.0 & & 1 & $11 / 06 / 89$ & $<$ & 10000.0 \\
\hline & 1 & Dichlorodif luoromethane & PPB & 10.0 & & I & $11 / 06 / 89$ & $<$ & 10.0 \\
\hline & 1 & Dielárin & PPB & .1 & & 1 & $11 / 06 / 89$ & $<$ & .1 \\
\hline & 1 & Diethyl phthalate & PPB & 10.0 & & 1 & $11 / 06 / 89$ & $<$ & 10.0 \\
\hline & 1 & Diethylstilbesterol & PPB & 200.0 & & 1 & $11 / 06 / 89$ & $<$ & 200.0 \\
\hline & 1 & Dihydrosafrole & PPB & 10.0 & & 1 & $11 / 06 / 89$ & $<$ & 10.0 \\
\hline & 1 & Dimethoate & PPB & 2.0 & & ? & $11 / 06 / 89$ & $<$ & 2.0 \\
\hline & 1 & Dimethyl phthalate & PPB & 10.0 & & 1 & $11 / 06 / 89$ & $<$ & 10.0 \\
\hline & 1 & Dinitrobenzene & PPB & 10.0 & & ] & $11 / 06 / 89$ & $<$ & 10.0 \\
\hline & 1 & Dinoseb & PPB & 10.0 & & 1 & $11 / 06 / 89$ & $<$ & 10.0 \\
\hline & 1 & Dioxane & PPB & 500.0 & & & $11 / 06 / 89$ & $<$ & 500.0 \\
\hline & 1 & Diphenylamine & PPB & 10.0 & . & 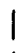 & $11 / 06 / 89$ & $<$ & 10.0 \\
\hline & 1 & Disulfoton & PPB & 2.0 & & & $11 / 06 / 89$ & $<$ & 2.0 \\
\hline & 1 & Endosulfan I & PPB & .1 & & & $11 / 06 / 89$ & $<$ & .1 \\
\hline & 1 & Endosulfan II & PPB & .1 & & 1 & $11 / 06 / 89$ & $<$ & .1 \\
\hline
\end{tabular}




\section{WHC-MR. 0229}

Well kesult Report

1100 Area Groundwater Monitoring

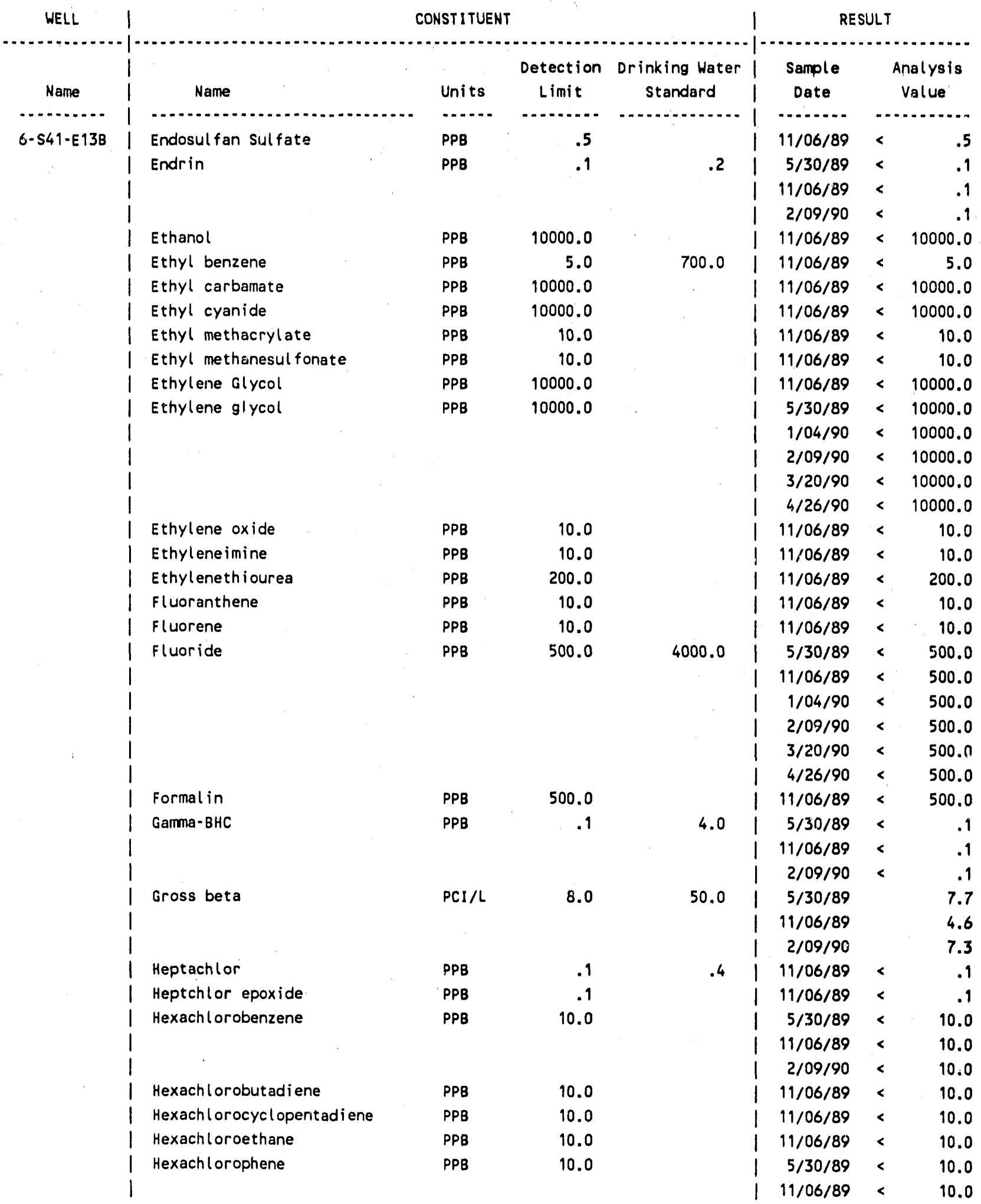


WHC.MR. 0229

$11 / 26 / 90$

Geosciences Group PARADOX Datebase

Well Result Report

Page 71

1100 Area Groundwater Monitoring

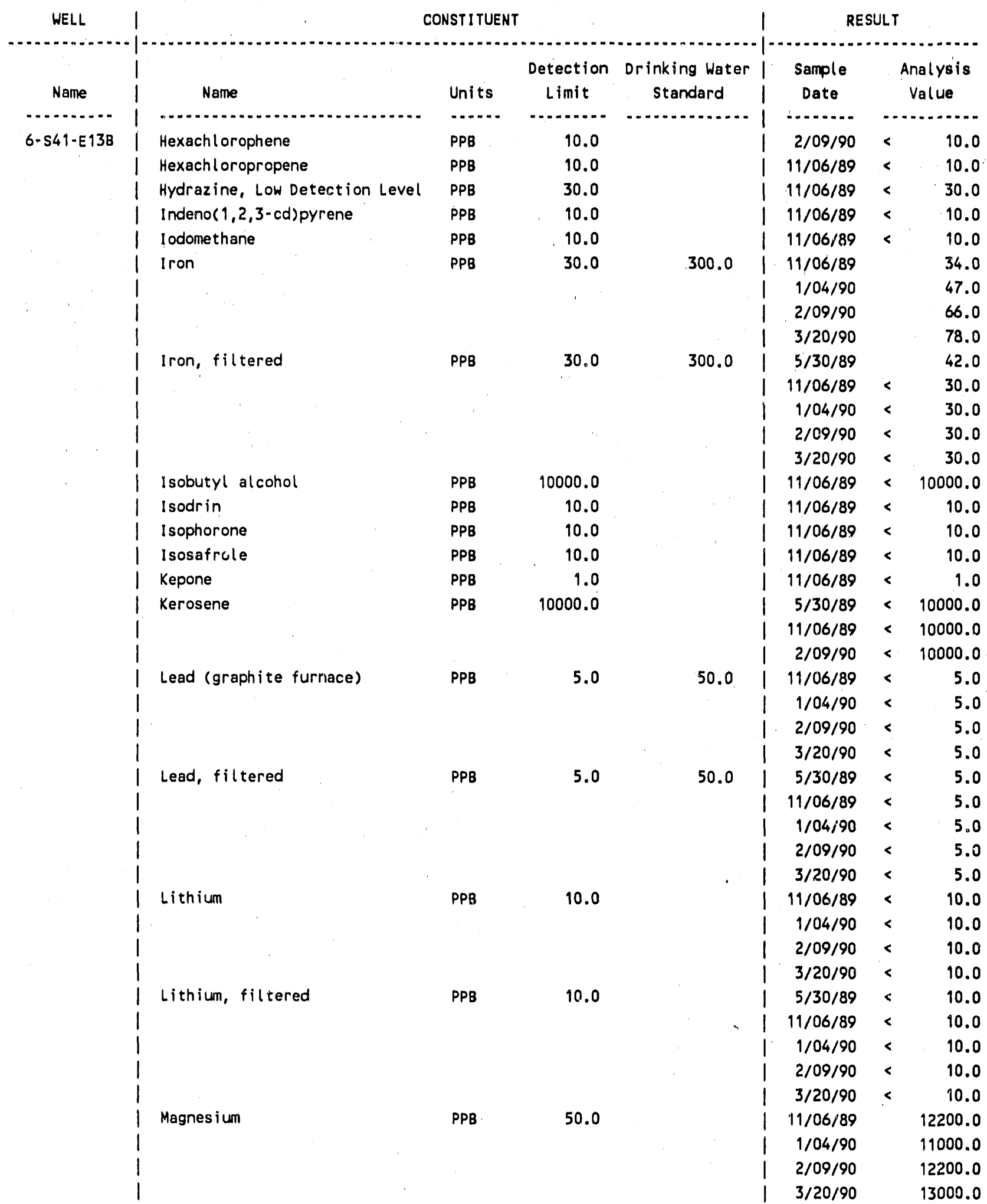




\section{WHC.MR. 0229}

$11 / 26 / 90$

Geosciences Group PARADOX Database

Well Result Report

Page 72

1100 Area Groundwater Monitoring

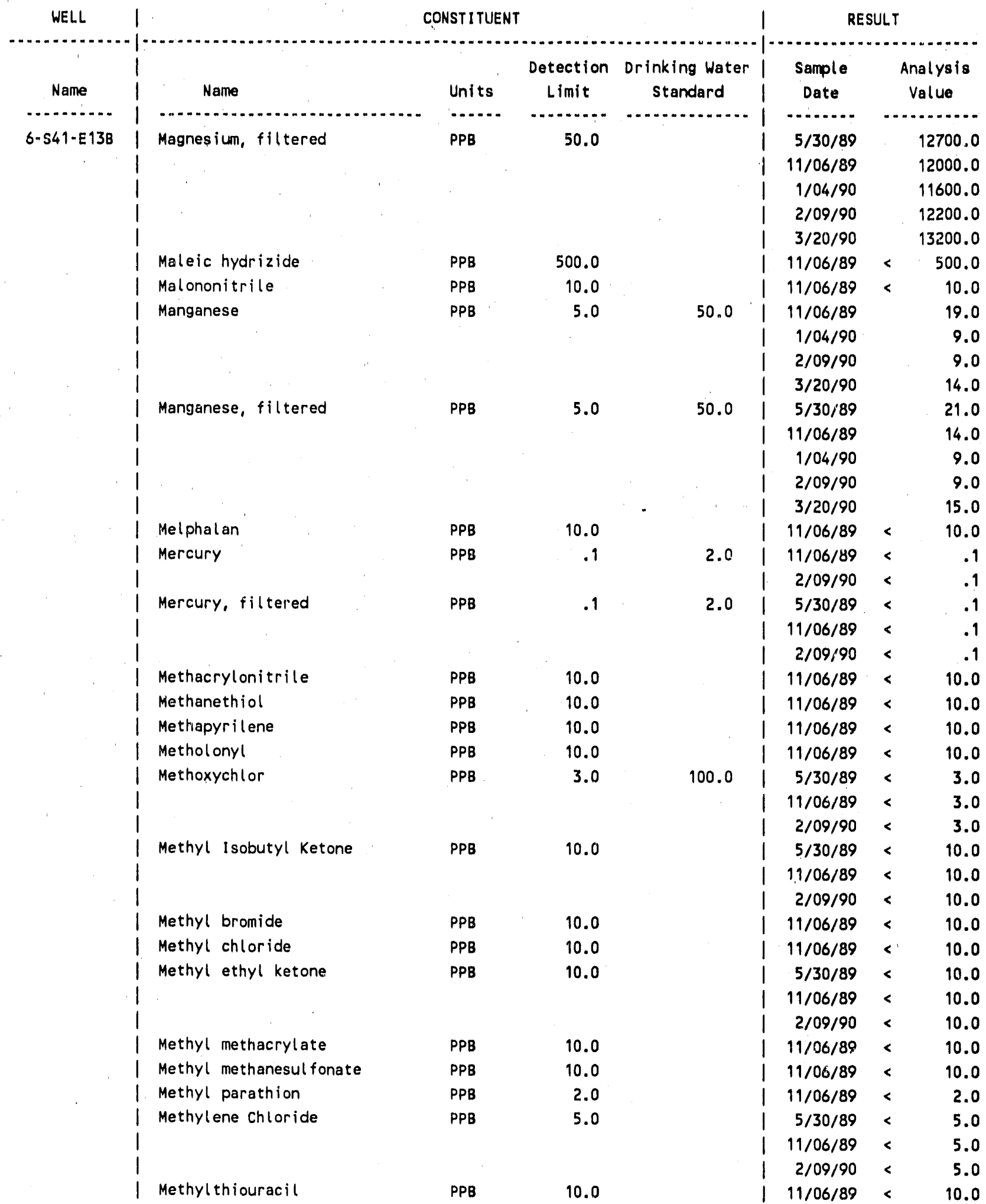




\section{WHC-MR. 0229}

Well Result Report

1100 Area Groundwater Monitoring

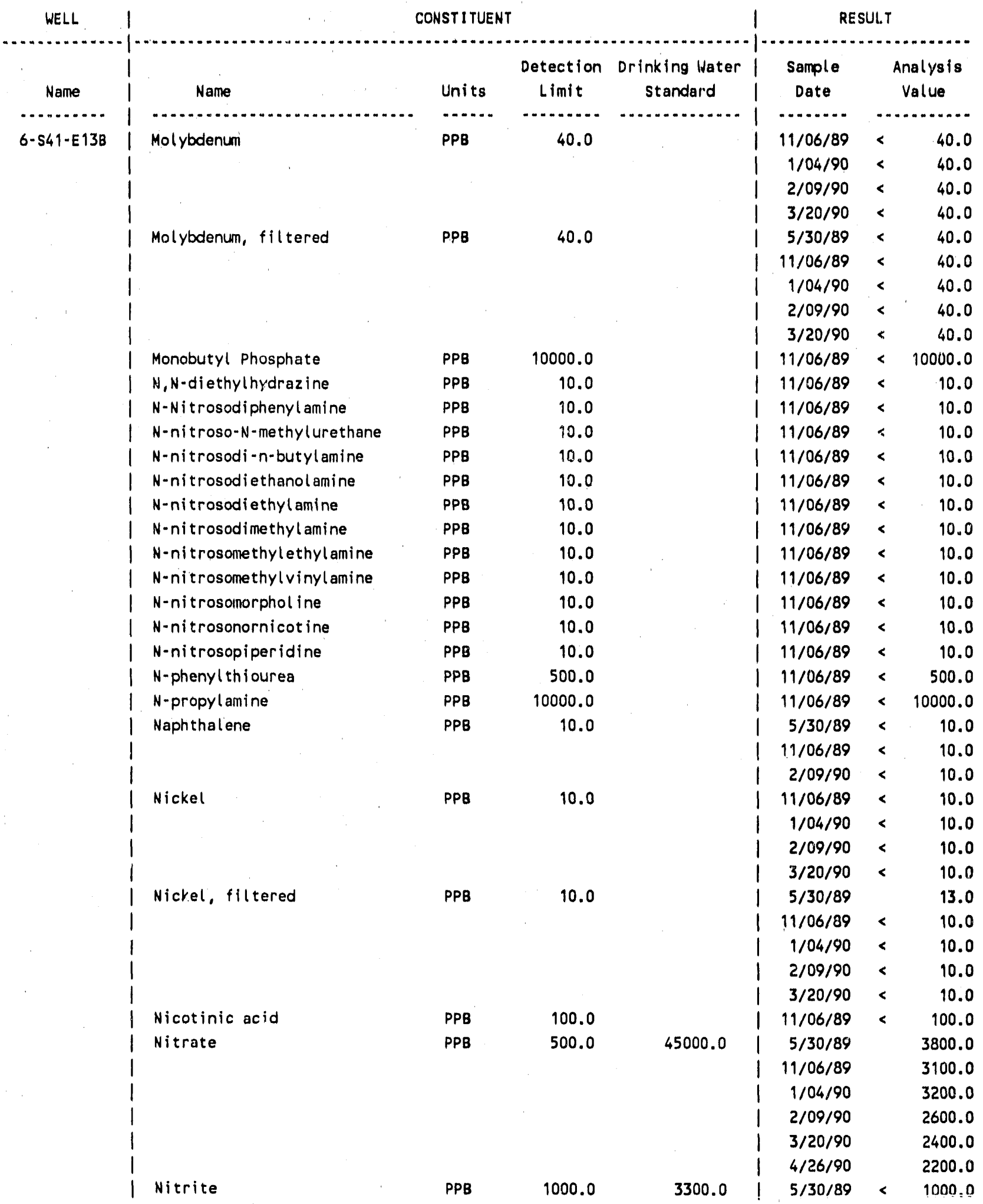


WHC-MR. 0229

$11 / 26 / 90$

Geosciences Group PARADOX Databuse

Page 74

Well Result Report

¿100 Area Groundwater Monitoring

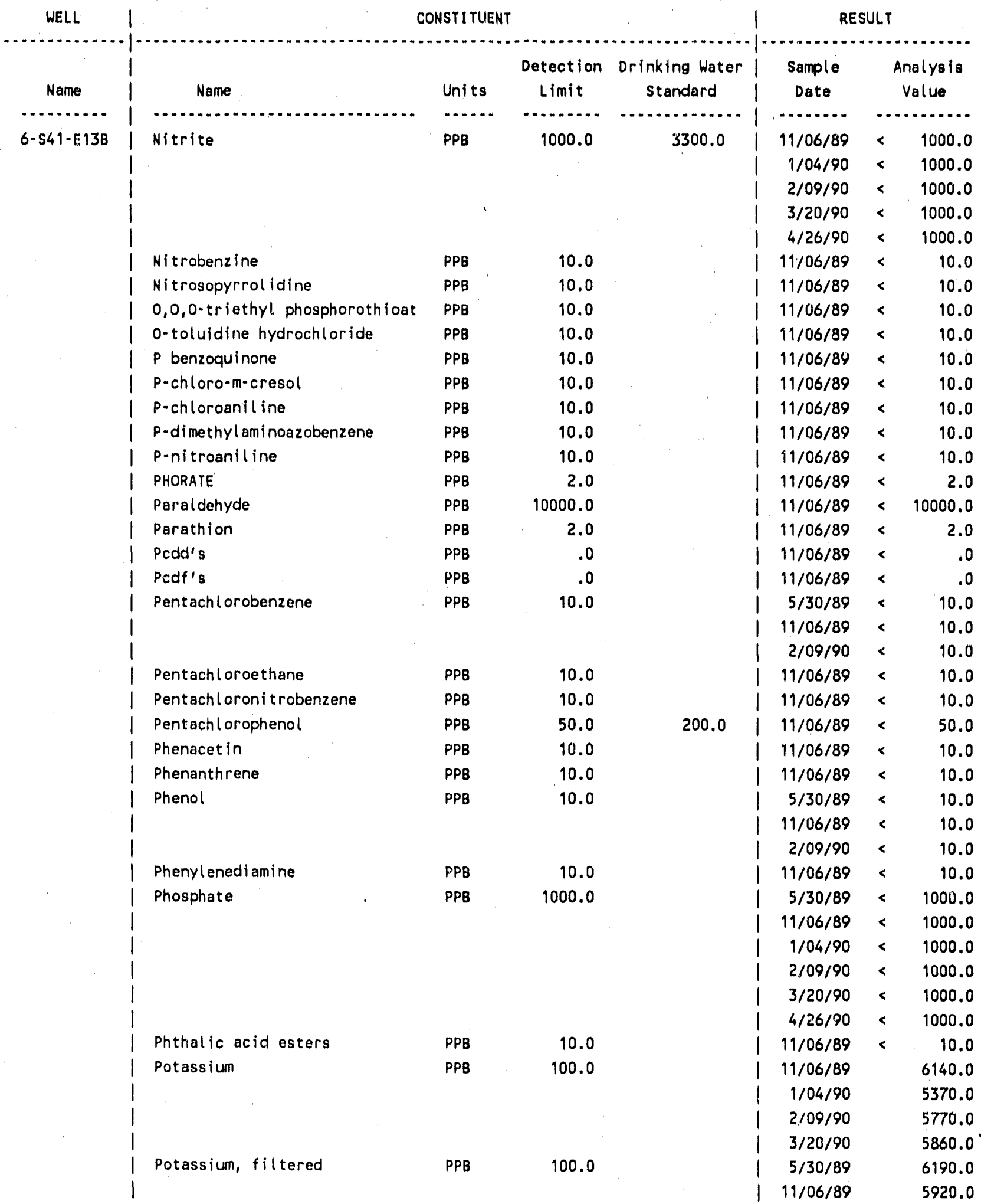


WHC-MR. 0229

$11 / 26 / 90$

Geosciences Group PARADOX Database

Well Result Report

1100 Area Groundwater Monitoring

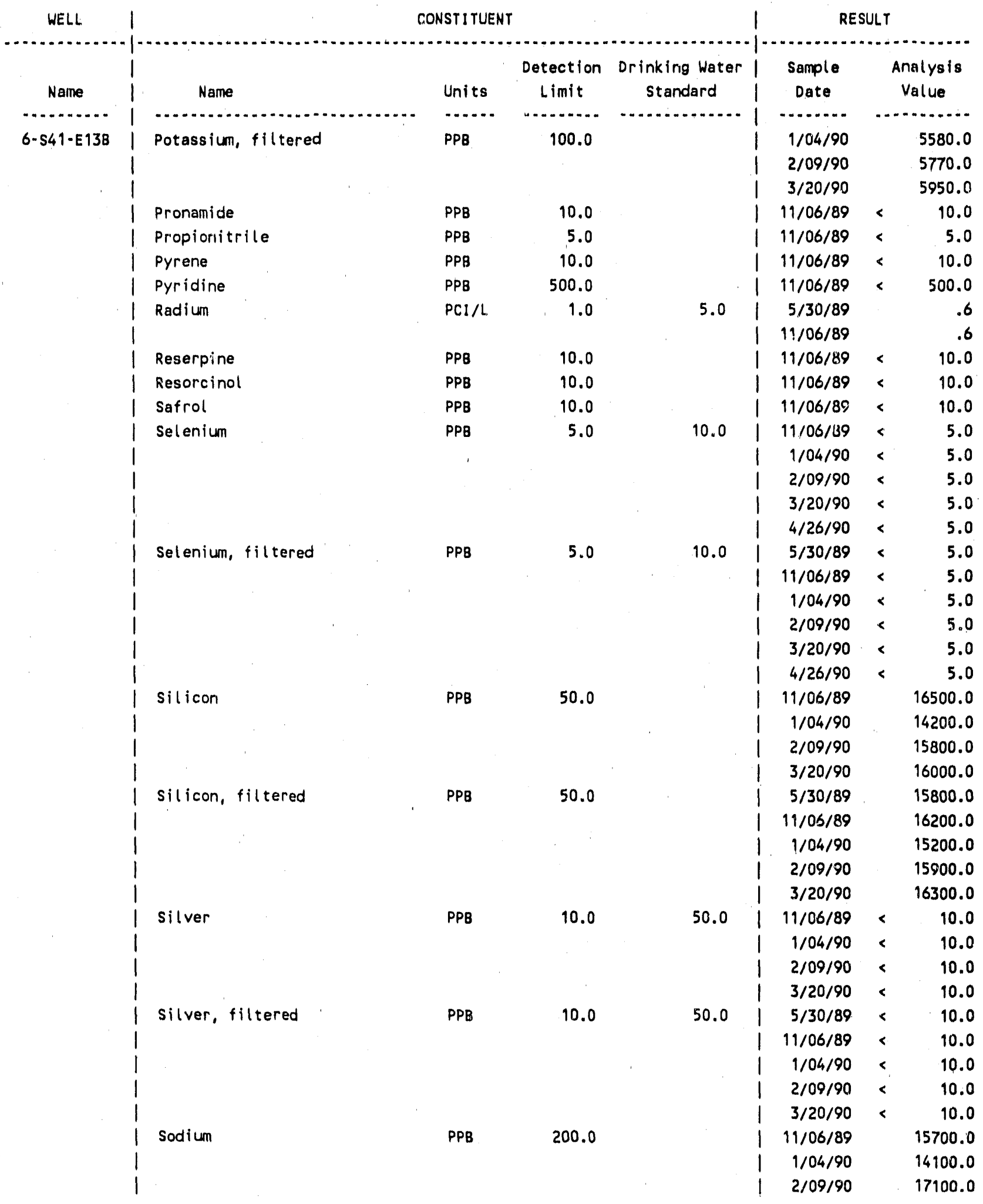


WHC-MR- 0229

$11 / 26 / 90$

Geosciences Group PARADOX Database

Well Result Report

Page 76

1100 Area Groundwater Monitoring

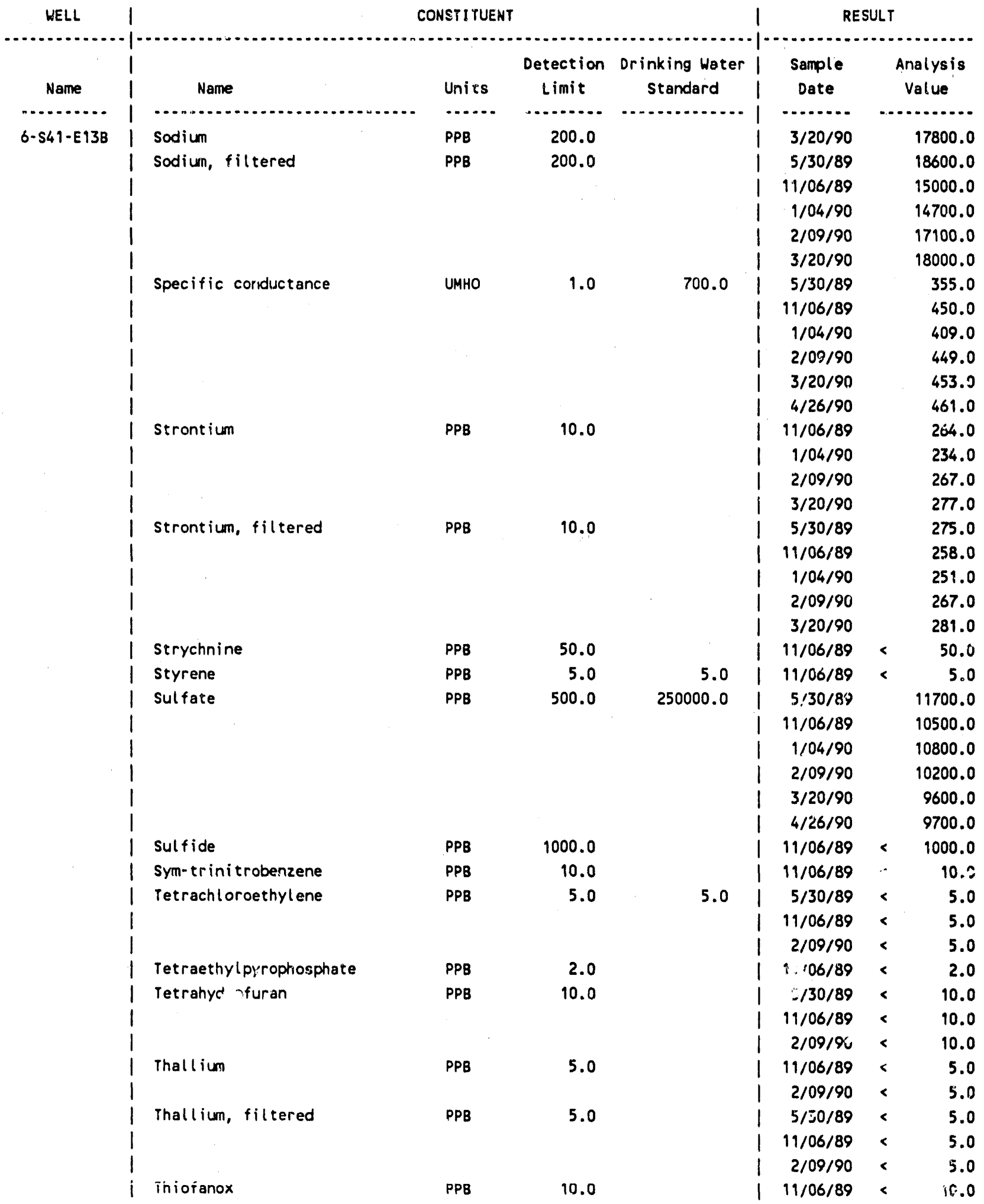


WHC-MR. 0229

Well Result Report

1100 Area Groundwater Monitoring

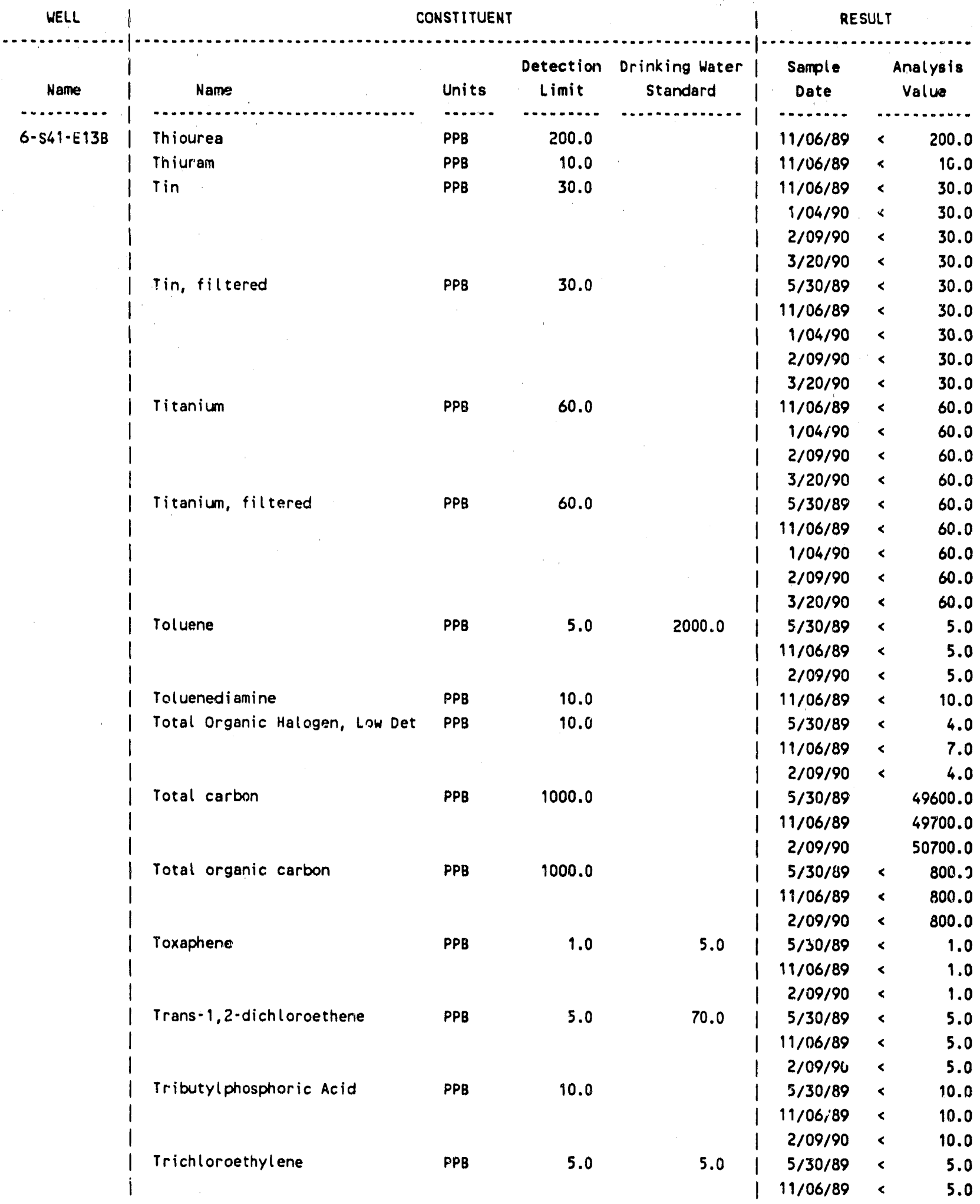




\section{WHC-MR. 0229}

$11 / 26 / 90$

Geosciences Group PARADOX Database

Well Result Report

1100 Area Groundwater Monitoring

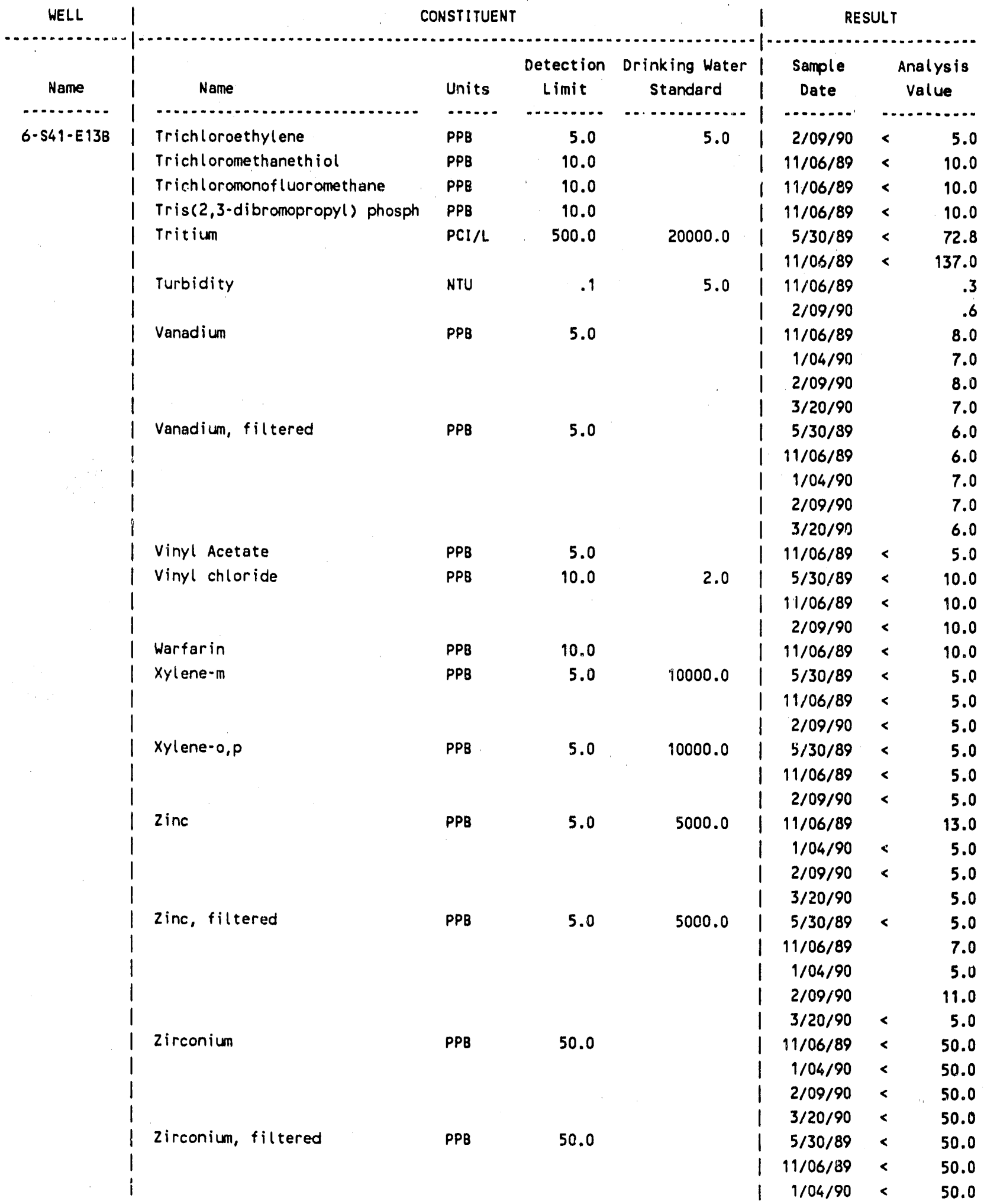


WHC-MR- 0229

$11 / 26 / 90$

Geosciences Group PARADOX Database

Well Result Report

1100 Area Groundwater Monitoring

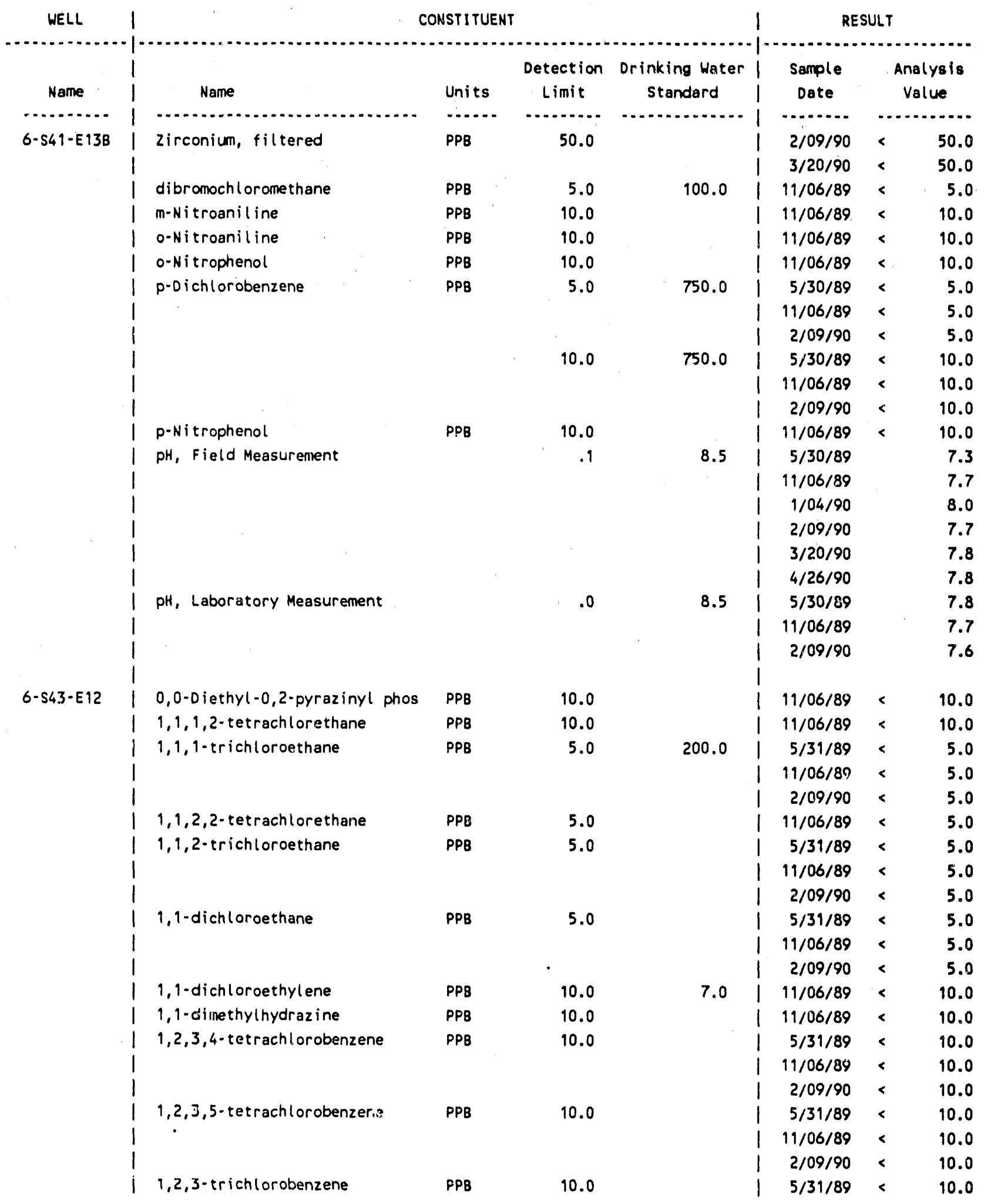




\section{WHC-MR- 0229}

Well Result Report

1100 Area Groundwater Monitoring

\begin{tabular}{|c|c|c|c|c|c|c|c|}
\hline \multirow{3}{*}{$\begin{array}{c}\text { WELL } \\
\text { Name }\end{array}$} & 1 & \multicolumn{3}{|c|}{ SONETITUENT } & \multicolumn{3}{|c|}{ RESULT } \\
\hline & 1 & Units & $\begin{array}{l}\text { Detection } \\
\text { Limit }\end{array}$ & $\begin{array}{c}\text { Drinking Water } \\
\text { standard }\end{array}$ & $\begin{array}{l}\text { Sample } \\
\text { Date }\end{array}$ & & $\begin{array}{l}\text { lalysis } \\
\text { lalue }\end{array}$ \\
\hline & ( & ....... & ............ & (.................. & ......... & & ........ \\
\hline \multirow[t]{44}{*}{$6-543-E 12$} & 1,2,3-trichlorobenzene & PPB & 10.0 & & $11 / 06 / 89$ & $<$ & 10.0 \\
\hline & $i$ & & & & 1. $2 / 09 / 90$ & $<$ & 10.0 \\
\hline & | 1,2,3-trichloropropane & PPB & 10.0 & & i 11/06/89 & $<$ & 10.0 \\
\hline & 1 1,2,4,5-tetrachlorobenzene & PPB & 10.0 & & 1 $5 / 31 / 89$ & $<$ & 10.0 \\
\hline & 1 & & & & I 11/06/89 & $<$ & 10.0 \\
\hline & 1 & & & & $12 / 09 / 90$ & $<$ & 10.0 \\
\hline & | 1,2,4-trichlorobenzene & PPB & 10.0 & & $5 / 31 / 89$ & $<$ & 10.0 \\
\hline & 1 & & & & I 11/06/89 & $<$ & 10.0 \\
\hline & 1 & & & & $2 / 09 / 90$ & $<$ & 10.0 \\
\hline & | 1,2-dibromo-3-chloropropane & PPB & 10.0 & .2 & | 11/06/89 & $<$ & 10.0 \\
\hline & 1 1,2-dibromoethane & PPB & 10.0 & & I $11 / 06 / 89$ & $<$ & 10.0 \\
\hline & | 1,2-dichlorobenzene & PPB & 10.0 & & 1. $5 / 31 / 89$ & $<$ & 10.0 \\
\hline & 1 & & & & I 11/06/89 & $<$ & 10.0 \\
\hline & 1 & & & & $1 \quad 2 / 09 / 90$ & $<$ & 10.0 \\
\hline & I 1,2-dichloroethane & PPB & 5.0 & 5.0 & $5 / 31 / 89$ & $<$ & 5.0 \\
\hline & 1 & & & & $11 / 06 / 89$ & $<$ & 5.0 \\
\hline & 1 & & & & $2 / 09 / 90$ & $<$ & 5.0 \\
\hline & I 9,2-dichloropropane & PPB & 5.0 & 5.0 & I 19/06/89 & $<$ & 5.0 \\
\hline & | 1,2-dimethylhydrazine & PPB & 10.0 & & $11 / 06 / 89$ & $<$ & 10.0 \\
\hline & | 1,2-diphenylhydrazine & PPB & 10.0 & & I 11/06/89 & $<$ & 10.0 \\
\hline & | 1,3,5-trichlorobenzene & PPB & 10.0 & & $5 / 31 / 89$ & $<$ & 10.0 \\
\hline & 1 & & & & $111 / 06 / 89$ & $<$ & 10.0 \\
\hline & 1 & & & & $1 \quad 2 / 09 / 90$ & $<$ & 10.0 \\
\hline & | 1,3-dichlorobenzene & PPB & 10.0 & & $5 / 31 / 89$ & $<$ & 10.0 \\
\hline & 1 & & & & I $11 / 06 / 89$ & $<$ & 10.0 \\
\hline & 1 & & & & $2 / 09 / 90$ & $<$ & 10.0 \\
\hline & 1,3-dichloropropene & PPB & 5.0 & & $11 / 06 / 89$ & $<$ & 5.0 \\
\hline & 1 1,4-dichloro-2-butene & PPB & 10.0 & & $111 / 06 / 89$ & $<$ & 10.0 \\
\hline & | 1,4-naphthoquinone & PPB & 10.0 & & | $11 / 06 / 89$ & $<$ & 10.0 \\
\hline & | 1-(o-chlorophenyl) thiourea & PPB & 200.0 & & $11 / 06 / 89$ & $<$ & 200.0 \\
\hline & 1 1-Butanol & PPB & 10000.0 & & I $11 / 06 / 89$ & $<$ & 10000.0 \\
\hline & | 1-Butynol & PPB & 10000.0 & & $11 / 06 / 89$ & $<$ & 10000.0 \\
\hline & | 1-Propanol & PPB & 10000.0 & & $11 / 06 / 89$ & $<$ & 10000.0 \\
\hline & | 1-acetyl-2-thiourea & PPB & 200.0 & & $11 / 06 / 89$ & $<$ & 200.0 \\
\hline & 1 1-chloro-2,3-epoxypropane & PPB & 10.0 & & $11 / 06 / 89$ & $<$ & 10.0 \\
\hline & I 1-naphthyl-2-thiourea & PPB & 200.0 & & $11 / 06 / 89$ & $<$ & 200.0 \\
\hline & | 1-naphthylamine & PPB & 10.0 & & $11 / 06 / 89$ & $<$ & 10.0 \\
\hline & | 2,3,4,6-tetrachlorophenol & PPB & 10.0 & & $11 / 06 / 89$ & $<$ & 10.0 \\
\hline & I $2,3,7,8 \mathrm{TCDD}$ & PPB & .0 & & $11 / 06 / 89$ & $<$ & .0 \\
\hline & I 2,4,5-T & PPB & 2.0 & & $5 / 31 / 89$ & $<$ & 2.0 \\
\hline & 1 & & & & $1 \quad 11 / 06 / 89$ & $<$ & 2.0 \\
\hline & 1 & & & & $1 \quad 2 / 09 / 90$ & $<$ & 2.6 \\
\hline & 2,4,5-TP silvex & PPB & 2.0 & 10.0 & $5 / 31 / 89$ & $<$ & 2.0 \\
\hline & $\mathrm{i}$ & & & & $11 / 06 / 89$ & $<$ & 2.0 \\
\hline
\end{tabular}


WHC-MR. 0229

$11 / 26 / 90$

Geosciences Group PARADOX Database

1100 Area Groundwater Monitoring

WELL $\quad 1, \quad 1 \%$ CONSTITUENT

(

\begin{tabular}{c|c} 
Name & Name \\
6-S43-E12 & $2,4,5-T P$ silvex
\end{tabular}

1 2,4,5-Trichlorophenol

2,4,6-trichlorophenol

\section{$P P B$}

I $2,4-0$

I

1

2,4-dichlorophenol

1 2,4-dinitrophenol

1 2,4-dinitrotoluene

I 2,6-dichlorophenol

1 2,6-dinitrotoluene

I 2-Hexanone

I 2-Methylnaphthalene

I 2-acetylaminofluorene

1. 2-chloroethyl vinyl ether

1 2-chloronaphthalene

1 2-chlorophenol

1 2-cyclohexyl-4,6-dinitrophenol

| 2-methyl-2-(methyt thio) propio

| 2-methylaziridine

I 2-methyllactonitrile

I 2-naphthylamine

I 2-picoline

1 2-propyn-1-ol

| 2-sec-butyl-4,6-dinitrophenol

1 3,31-Dichlorobenzidine

| 3,3'-dimethoxybenzidine

1 3,3'-dimethylbenzidine

I 3-chloropropionitrile

I 3-methylchol anthrene

I 4,4'-methylenebis (2-chloroanil

1 4,6-dinitro-o-cresol and salts

I 4-Nitroquinoline 1-oxide

| 4-aminobyphenyl

1 4-bromophenyt phenyt ether

| 5-(aminomethyl)-3-isoxazolol

15-nitro-o-toluidine

I 7,12-dimethylbenz [a] anthracene

| 7H-dibenzo[c,g]carbazole

I Acenaphthal ene

I Acenaphthene

I Acetone - by $A B N$

1 Acetone by VOA

i
....

Limit

2.0

10.0

10.0

2.0

PPB

PPB

$P P B$

PPB

PPB

PPB

PPB

$P P B$

PPB

$P P B$

PPB

PPB

PPB

PPB

PPB

PPB

PPB

PPB

PPB

PPB

PPB

PP8

PPB

PPB

PPB

PPB

PPB

PPR

$P P B$

PPB

PPB

PPB

PPB

PPB

PPB

$P P B$

PPB

PPB
10.0

10.0

10.0

10.0

10.0

50.0

10.0

10.0

5.0

10.0

10.0

10.0

$10 . .0$

10.0

10.0

10.0

10.0

10000.0

10.0

10.0

10.0

10.0

10000.0

10.0

10.0

10.0

10.0

10.0

10.0

10.0

$: 0.0$

10.0

10.0

10.0

10.0

10.0

10.0

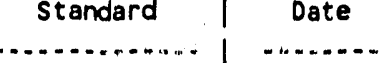

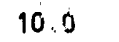

| $11 / 06 / 89$

| $11 / 06 / 89$

$100.0 \mid 5 / 3 i / 89$

$111 / 06 / 89<$

| $2 / 09 / 90<$

$11 / 06 / 89$

I $11 / 06 / 89$

| $11 / 06 / 89$

| $11 / 06 / 89$

| 11/06/89

$11 / 06 / 89$

| $11 / 06 / 89$

$11 / 06 / 89$

| $11 / 06 / 89$

| $11 / 06 / 89$

| $11 / 06 / 89$

| $11 / 06 / 89$

| $11 / 06 / 89$

| 11/06/89

| $11 / 06 / 89$

| $11 / 06 / 89$

| $11 / 06 / 89$

| $11 / 06 / 89$

| 11/06/89

| $11 / 06 / 89$

| $11 / 06 / 89$

| $11 / 06 / 89$

$11 / 06 / 89$

| 11/06/89

I 11/06/89

| $11 / 06 / 89$

| $11 / 06 / 89$

| $11 / 06 / 89$

| $11 / 06 / 89$

| $11 / 06 / 89$

| 11/06/89

| $11 / 06 / 89$

| $11 / 06 / 89$

| $11 / 06 / 89$

| $11 / 06 / 89$

| $11 / 06 / 89$

| 5/31/89

| 11/06/89
2.0

10.0

10.0

2.0

2.0

2.0

10.0

10.0

10.0

10.0

10.0

50.0

10.0

10.0

5.0

10.0

10.0

10.0

10.0

10.0

10.0

10.0

10.0

10000.0

10.0

10.0

10.0

10.0

10000.0

10.0

10.0

10.0

10.0

10.0

10.0

10.0

10.0

10.0

10.0

10.0

10.0

10.0

10.0

10.0 


\section{WHC-MR- 0229}

Well Result Report

1100 Area Groundwater Monitoring

\begin{tabular}{|c|c|c|c|c|c|c|c|c|}
\hline \multirow{2}{*}{\multicolumn{2}{|c|}{ WELL }} & \multicolumn{4}{|c|}{ CONSTITUENT } & \multicolumn{3}{|c|}{ RESULT } \\
\hline & & Name & Units & $\begin{array}{l}\text { Detection } \\
\text { Limit }\end{array}$ & $\begin{array}{c}\text { Drinking Water } \\
\text { standard }\end{array}$ & $\begin{array}{l}\text { Sample } \\
\text { Date }\end{array}$ & & $\begin{array}{l}\text { Analysis } \\
\text { Value }\end{array}$ \\
\hline 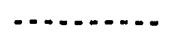 & 1 & (n) & $\cdots$ & $\ldots \ldots$ & 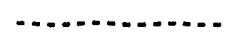 & | $\cdots m \ldots$ & & $\ldots . . . .$. \\
\hline \multirow[t]{44}{*}{$6-S 43-E 12$} & i & Acetone by VOA & PPB & 10.0 & & | $2 / 09 / 90$ & $<$ & 10.0 \\
\hline & 1 & Acetonitrile & PPB & 10.0 & & $111 / 06 / 89$ & $<$ & 10.0 \\
\hline & I & Acetophenone & PPB & 10.0 & & $11 / 06 / 89$ & $<$ & 10.0 \\
\hline & 1 & Acrolein & PPB & 10.0 & & | 11/06/89 & $<$ & 10.0 \\
\hline & 1 & Acrylamide & PPB & $10000: 0$ & & $111 / 06 / 89$ & $<$ & 10000.0 \\
\hline & 1 & Acrylonitrile & PPB & 10.0 & & $11 / 06 / 89$ & $<$ & 10.0 \\
\hline & I & Aldrin & PPB & .1 & & $11 / 06 / 89$ & $<$ & .1 \\
\hline & I & Alkalinity & & 20000.0 & & $15 / 31 / 89$ & & 205000.0 \\
\hline & 1 & & & & & $191,06 / 89$ & & 107000.0 \\
\hline & I & Allyl Chloride & PPB & 100.0 & & $111 / 06 / 89$ & $<$ & 100.0 \\
\hline & I & Allyl alcohol & PPB & 10000.0 & & $11 / 06 / 89$ & $<$ & 10000.0 \\
\hline & 1 & Alpha, High Detection Level & $\mathrm{PCI} / \mathrm{L}$ & 4.0 & 15.0 & $5 / 31 / 89$ & & 3.6 \\
\hline & 1 & & & & & $11 / 06 / 89$ & & 2.6 \\
\hline & 1 & & & & & $1 \quad 2 / 09 / 90$ & & 3.7 \\
\hline & I & Alpha, alpha-dimethylphenethyla & PPB & 10.0 & & | $11 / 06 / 89$ & $<$ & 10.0 \\
\hline & 1 & Alpha-BHC & PPG & .1 & 4.0 & $5 / 31 / 89$ & $<$ & .1 \\
\hline & 1 & & & & & $111 / 06 / 89$ & $<$ & .1 \\
\hline & 1 & & & & & 1 $2 / 09 / 90$ & $<$ & .1 \\
\hline & 1 & Aluminum & PPB & 150.0 & 50.0 & $111 / 06 / 89$ & $<$ & 150.0 \\
\hline & 1 & & & & & $12 / 09 / 20$ & $<$ & 150.0 \\
\hline & 1 & Aluminum, filtered & PPB & 150.0 & 50.0 & | 5/31/89 & $<$ & 150.0 \\
\hline & 1 & & & & & | $11 / 06 / 89$ & $<$ & 150.0 \\
\hline & 1 & & & & & $2 / 89 / 90$ & 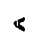 & 150.0 \\
\hline & 1 & Amitrole & PPB & 10.0 & & $11 / 06 / 89$ & $<$ & 10.0 \\
\hline & I & Ammonium ion & PPB & 50.0 & & $11 / 06 / 89$ & $<$ & 50.0 \\
\hline & 1 & Aniline & PPB & 10.0 & & $11 / 06 / 89$ & $<$ & 10.0 \\
\hline & 1 & Anthracene & PPB & 10.0 & & | 11/06/89 & $<$ & 10.0 \\
\hline & 1 & Ant imony & PPB & 100.0 & & | 11/06/89 & $<$ & 100.0 \\
\hline & 1 & & & & & | 2/09/90 & $<$ & 100.0 \\
\hline & 1 & Antimony, filtered & PPB & 100.0 & & $5 / 31 / 89$ & $<$ & 100.0 \\
\hline & 1 & & & & & $111 / 06 / 89$ & $<$ & 100.0 \\
\hline & 1 & & & & & | 2/09/90 & $<$ & 100.0 \\
\hline & 1 & Aramite & PPB & 10.0 & & I 11/06/89 & $<$ & 10.0 \\
\hline & 1 & Arochlor 1016 & PPB & 1.0 & .5 & $5 / 31 / 89$ & $<$ & 1.0 \\
\hline & 1 & & & & & | 11/06/89 & $<$ & 1.0 \\
\hline & 1 & & & & & | $2 / 09 / 90$ & $<$ & 1.0 \\
\hline & 1 & Arochlor 1221 & PPB & 1.0 & .5 & | $5 / 31 / 89$ & $<$ & 1.0 \\
\hline & 1 & & & & & | 11/06/89 & $<$ & 1.0 \\
\hline & 1 & & & & & I $2 / 09 / 90$ & $<$ & 1.0 \\
\hline & 1 & Arochlor 1232 & PPB & 1.0 & .5 & | 5/31/89 & $<$ & 1.0 \\
\hline & 1 & & & & & | 11/06/89 & $<$ & 1.0 \\
\hline & 1 & & & & & $2 / 09 / 90$ & $<$ & 1.0 \\
\hline & 1 & Arochlor 1242 & PPB & 1.0 & .5 & $5 / 31 / 89$ & $<$ & 1.0 \\
\hline & $\mathrm{i}$ & & & & & I 11/06/89 & $<$ & 1.0 \\
\hline
\end{tabular}




\section{WHC-MR- 0229}

$11 / 26 / 90$

Geosciences Group PARADOX Database

Well Result Repurt

Page 83

1100 Area Groundwater Monitoring

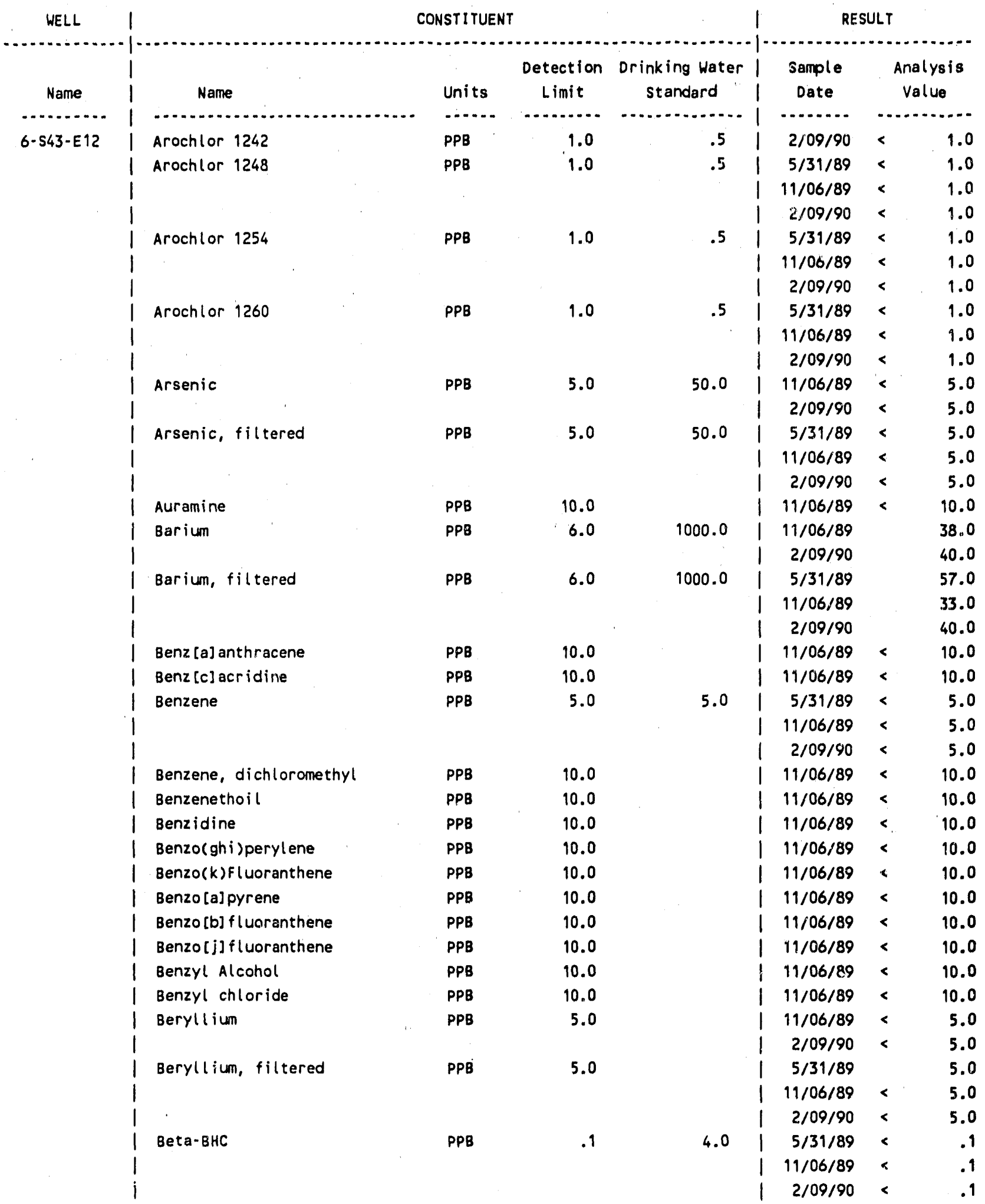




\section{WHC-MR. 0229}

Well Result Report

1100 Area Groundwater Monitoring

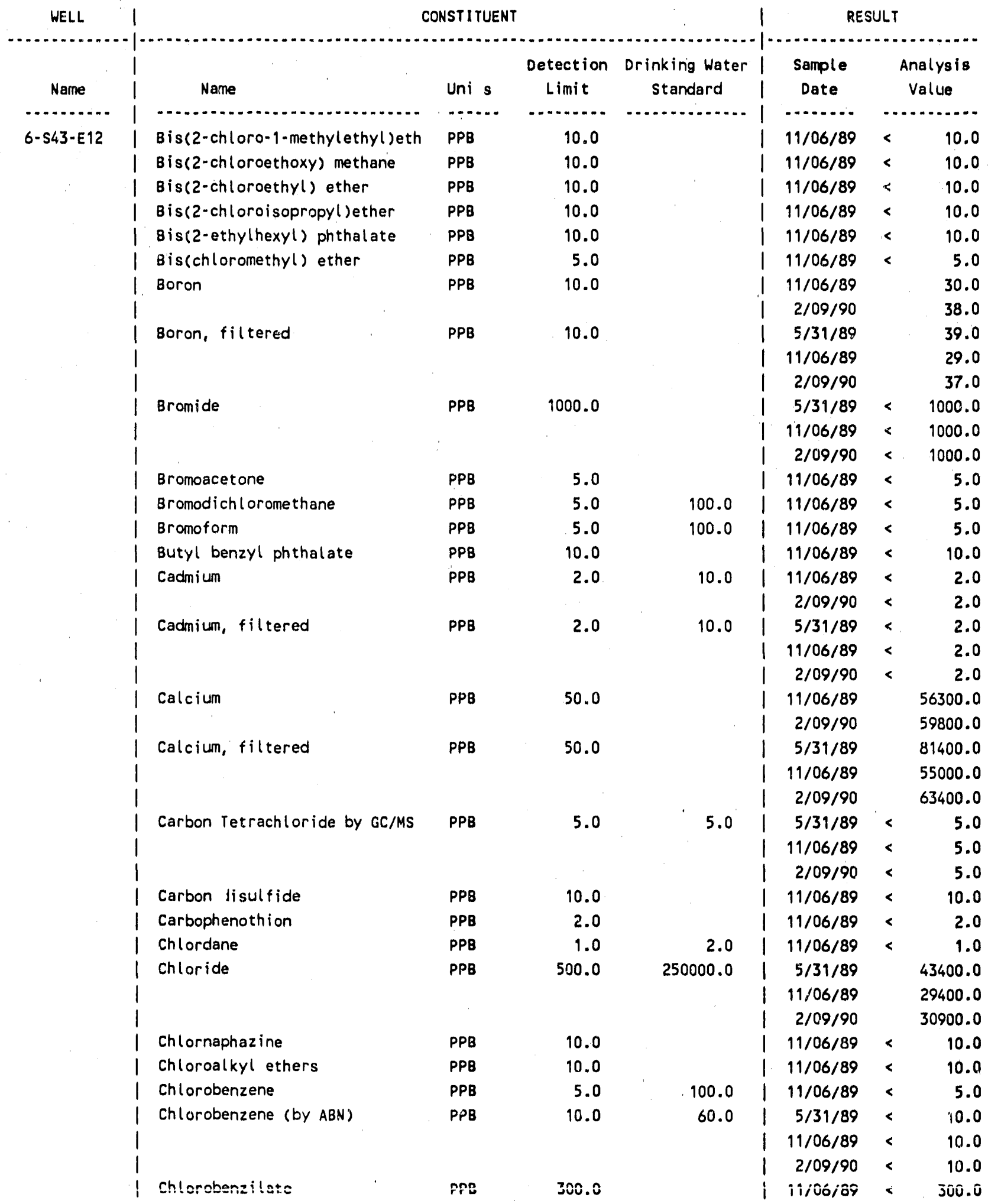




\section{WHC-MR- 0229}

Well Result Report

1100 Area Groundwater Monitoring

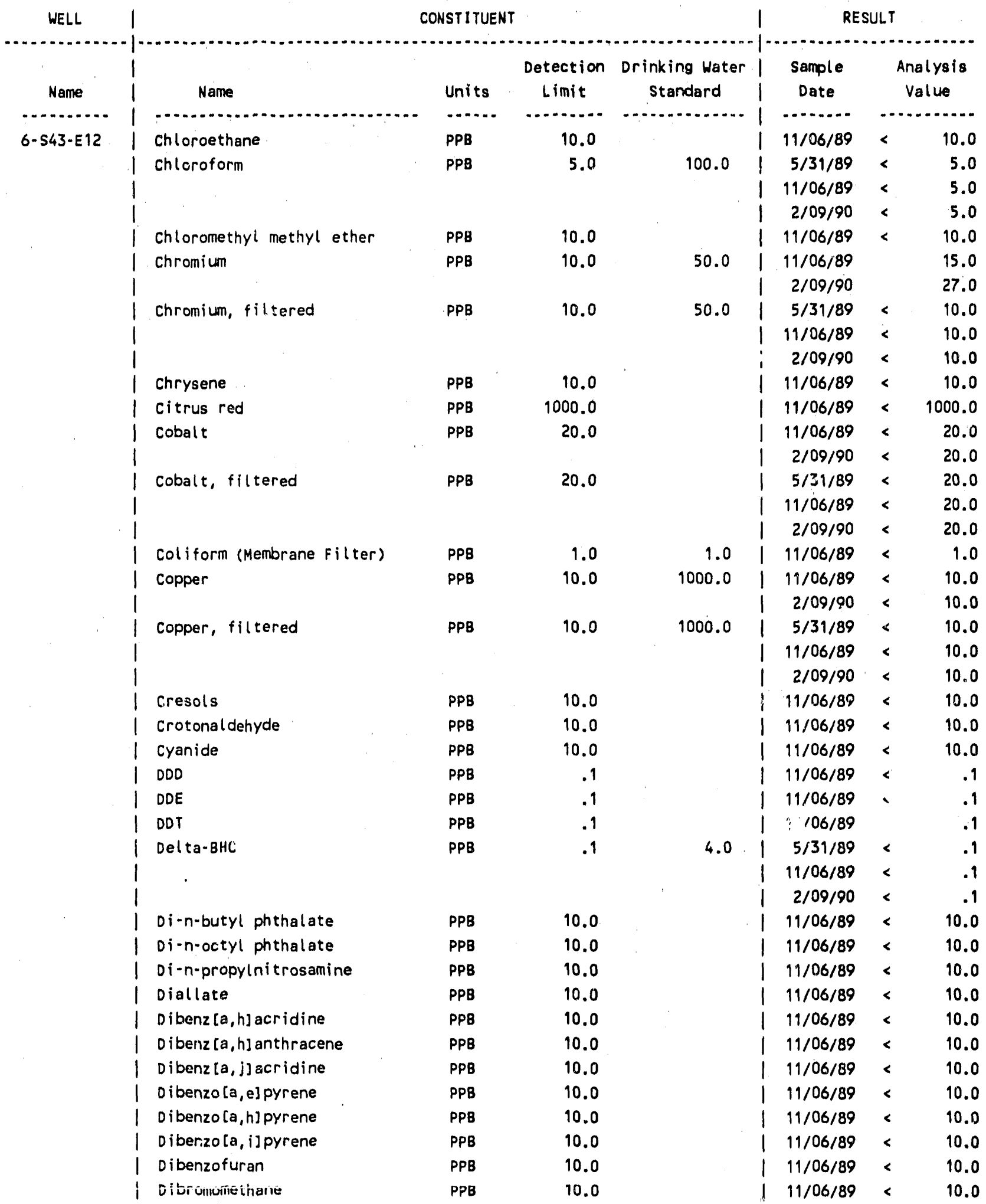


WHC-MR- 0229

$11 / 26 / 90$

Geosciences Group PARADOX Database

Well Result Report

Page 86

1100 Area Groundwater Monitoring

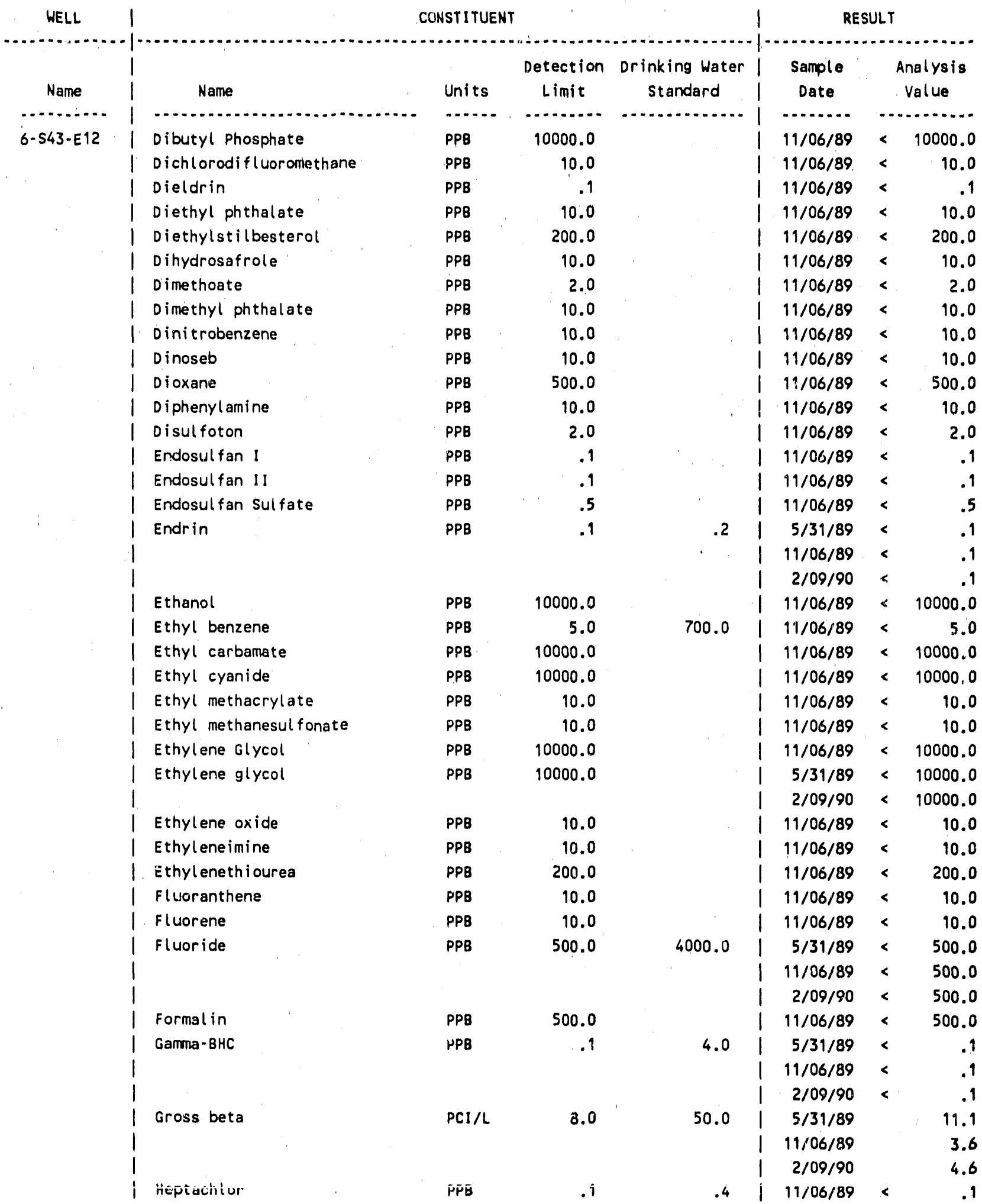


WHC-MR- 0229

$11 / 26 / 90$

Geosciences Group PhRADOX Database

Well Result Report

Page 87

1100 Area Groundwater Monitoring

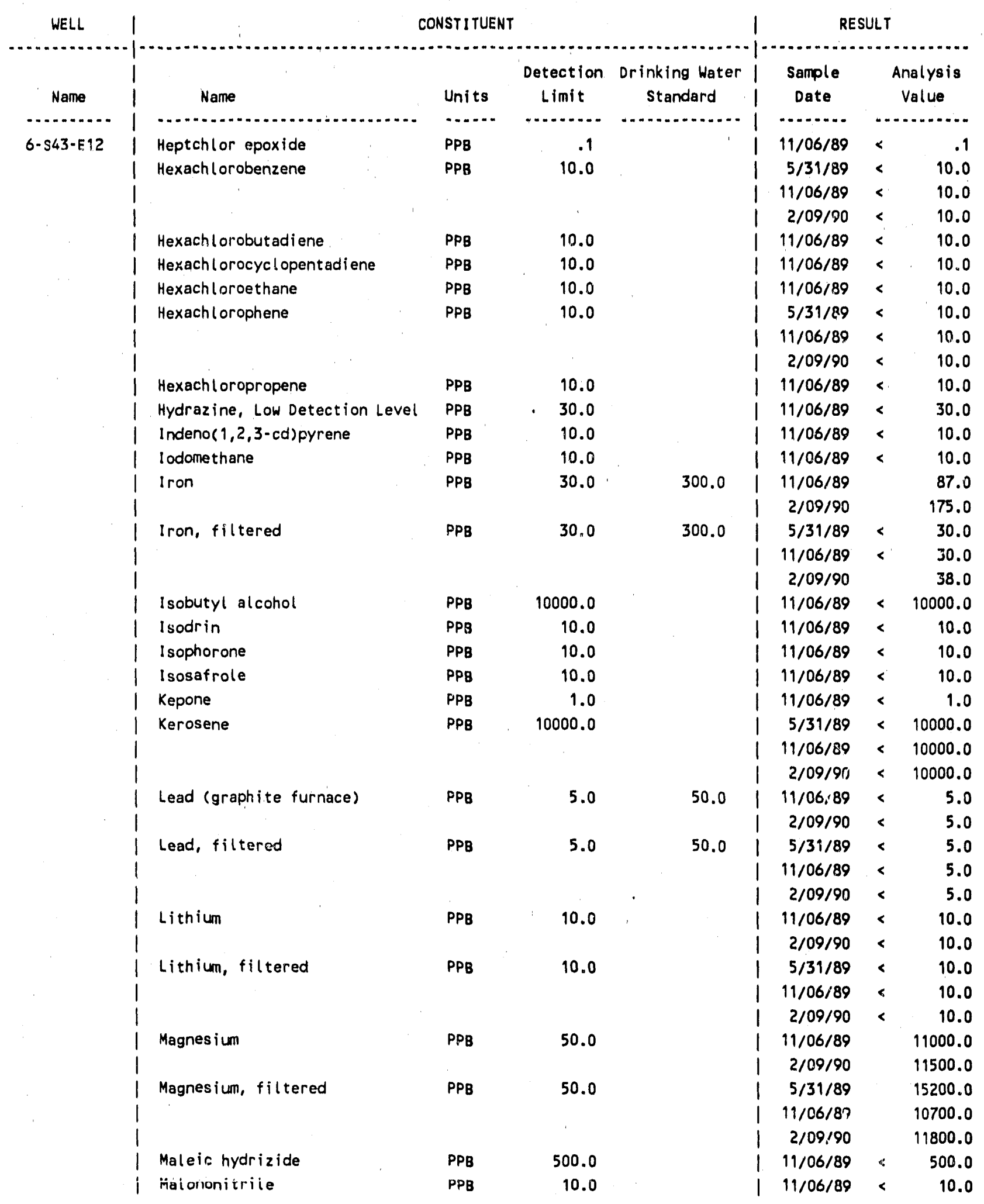


WHC-MR. 0229

$11 / 26 / 90$

Geosciences Group PARADOX Database

Page 88

Well Result Report

1100 Area Groundwater Monitoring

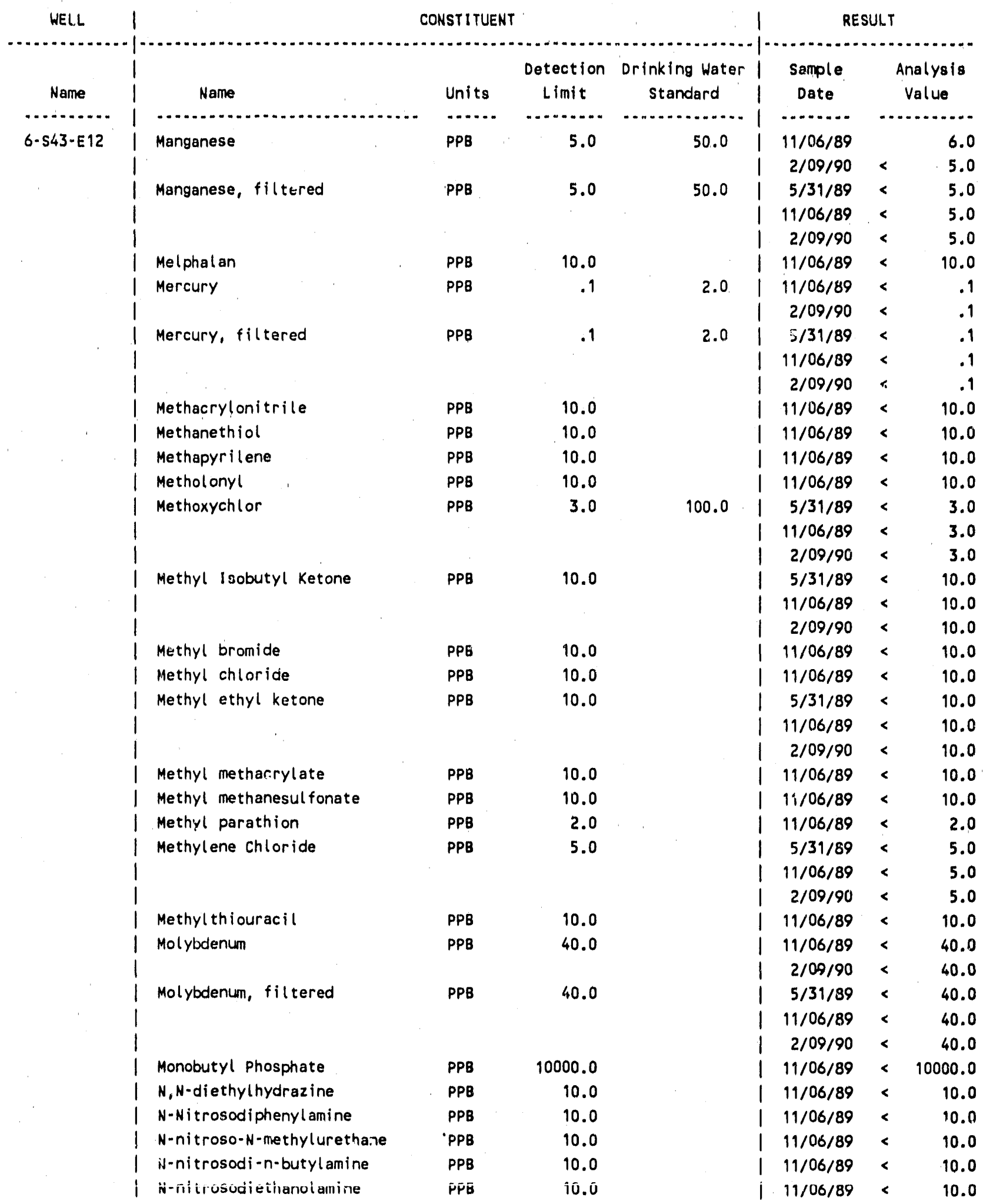




\section{WHC-MR- 0229}

$11 / 26 / 90$

Geosciences Group PARADOX Database

Well Result Report

Page 89

1100 Area Groundwater Monitoring

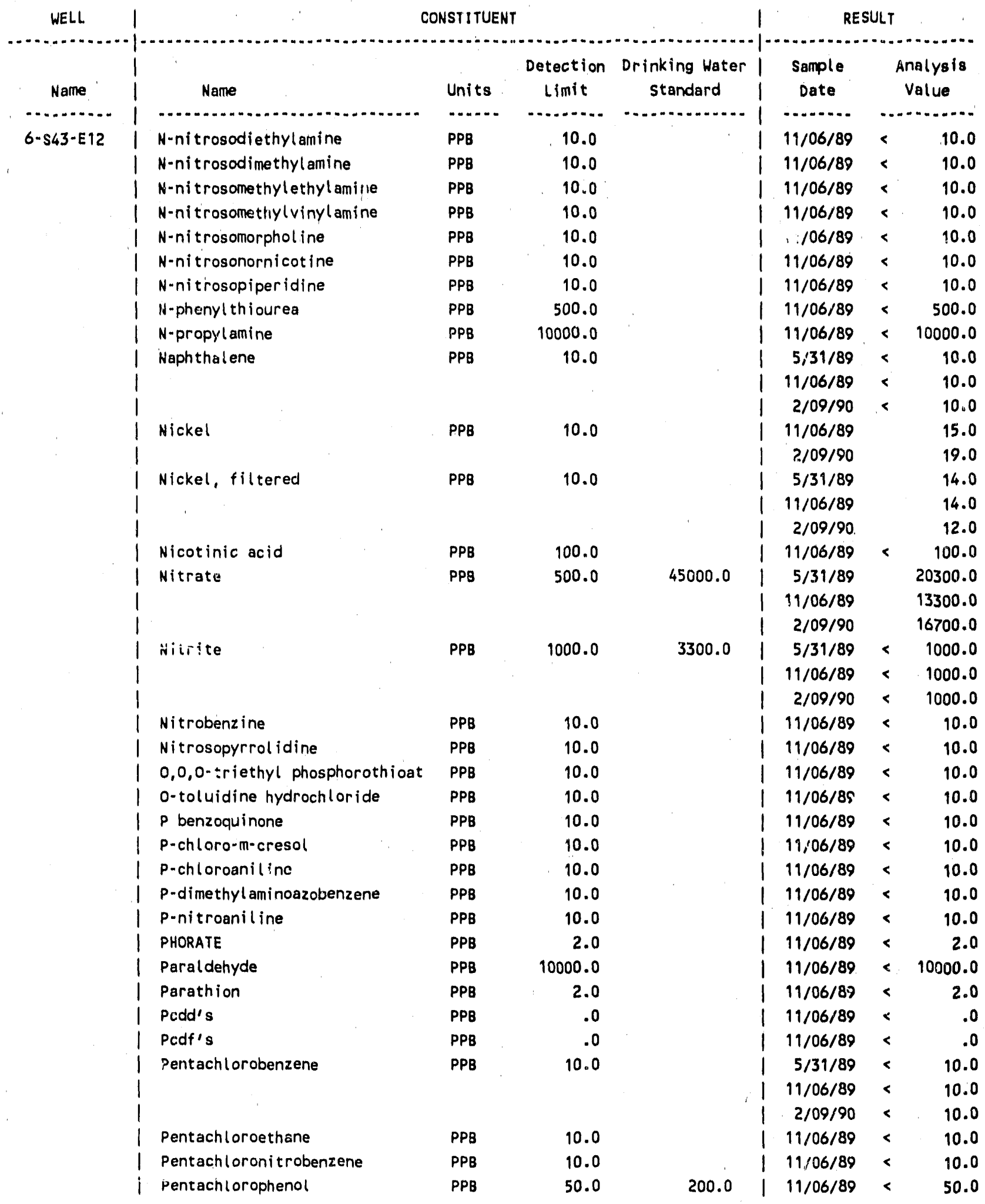


WHC.MR. 0229

$11 / 26 / 90$

Geosciences Group PARADOX Database

Hell Result Report

Page 90

1100 Area Groundwater Monitoring

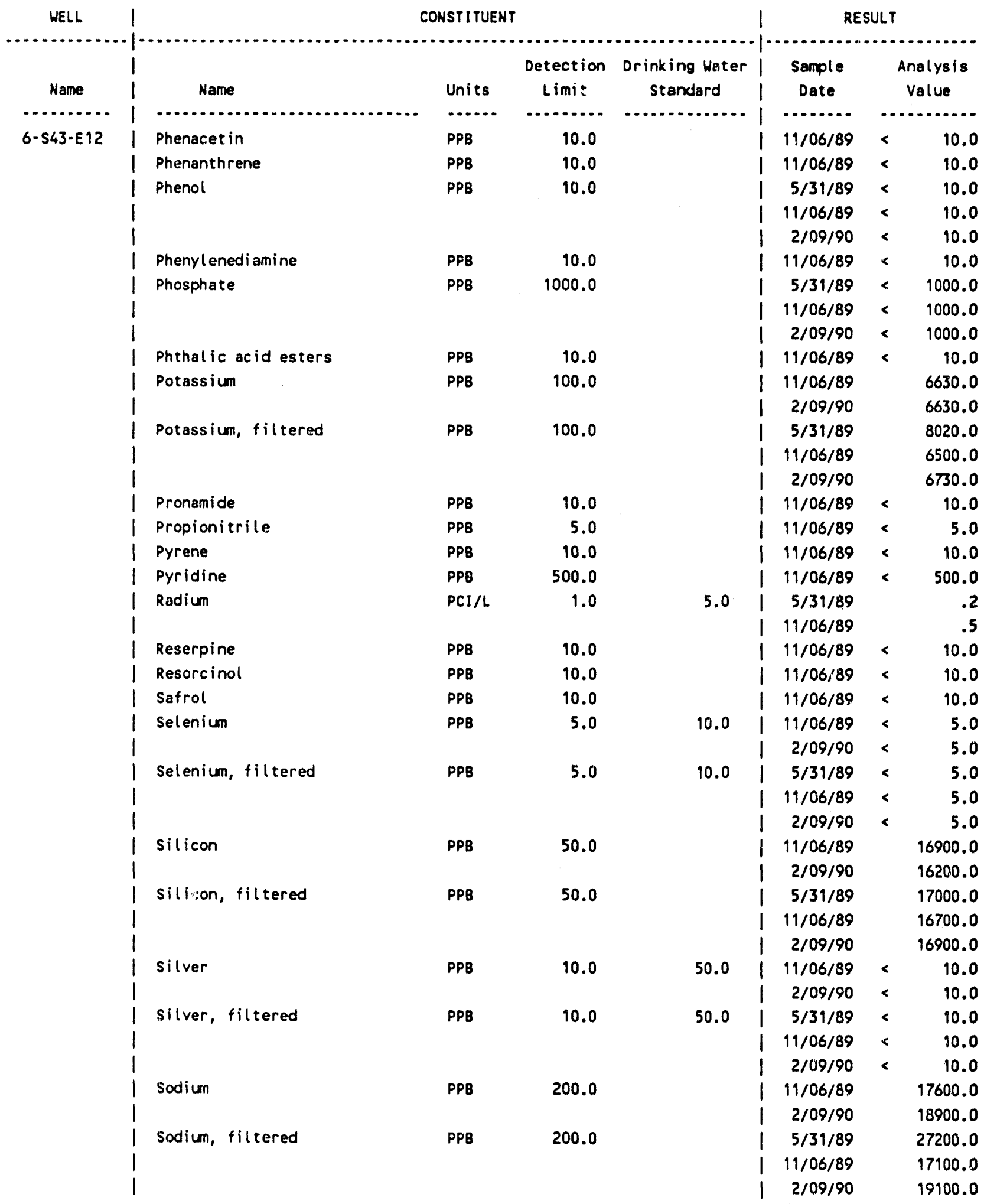


WHC-MR. 0229

$11 / 26 / 90$

Geosciences Group PARADOX Database

Well Result Report

Page 91

1100 Area Groundwater Monitoring

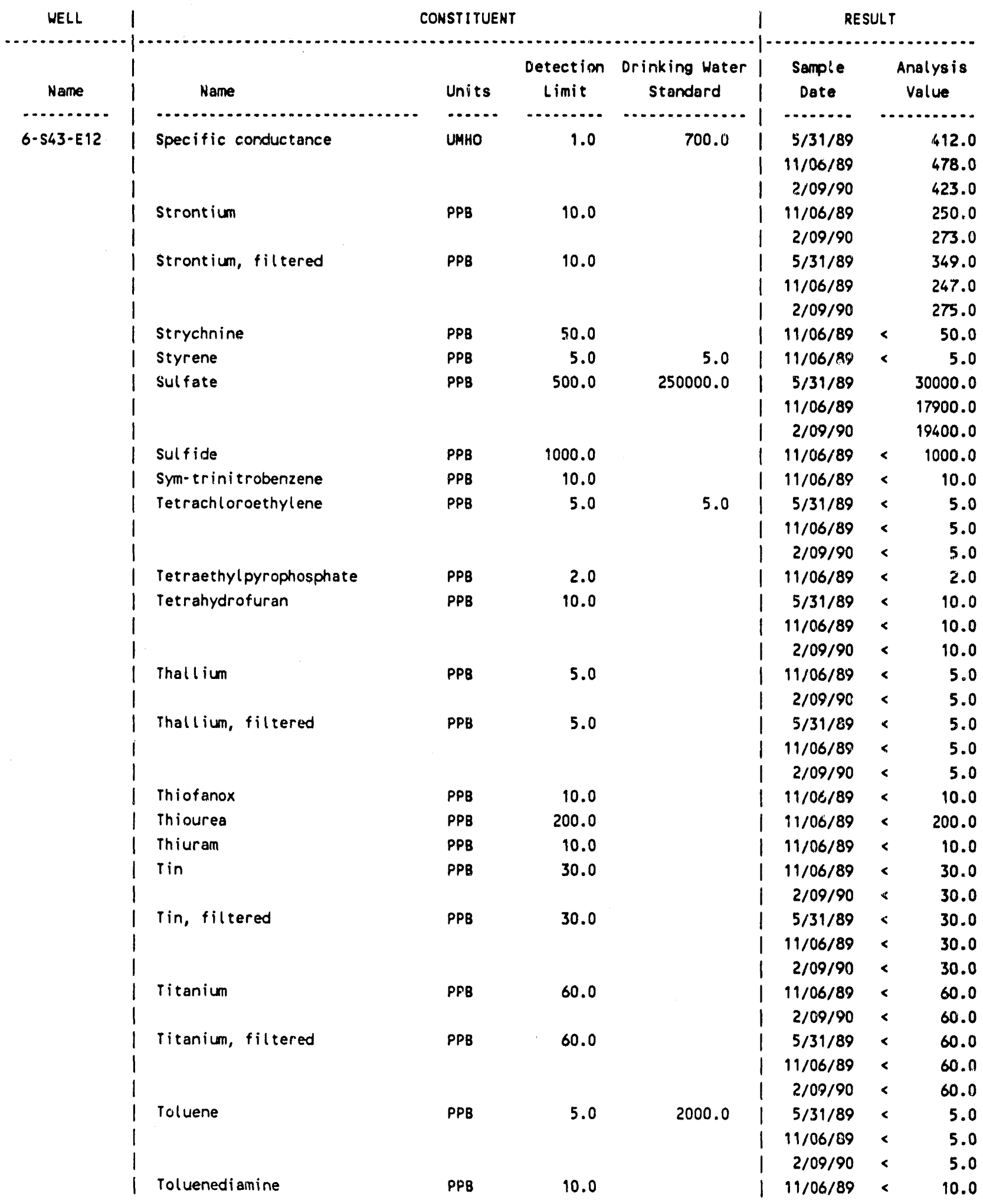


WHC.MR- 0229

$19 / 26 / 90$

Geosciences Group PARADOX Database

Well Result Report

1100 Area Groundwater Monitoring

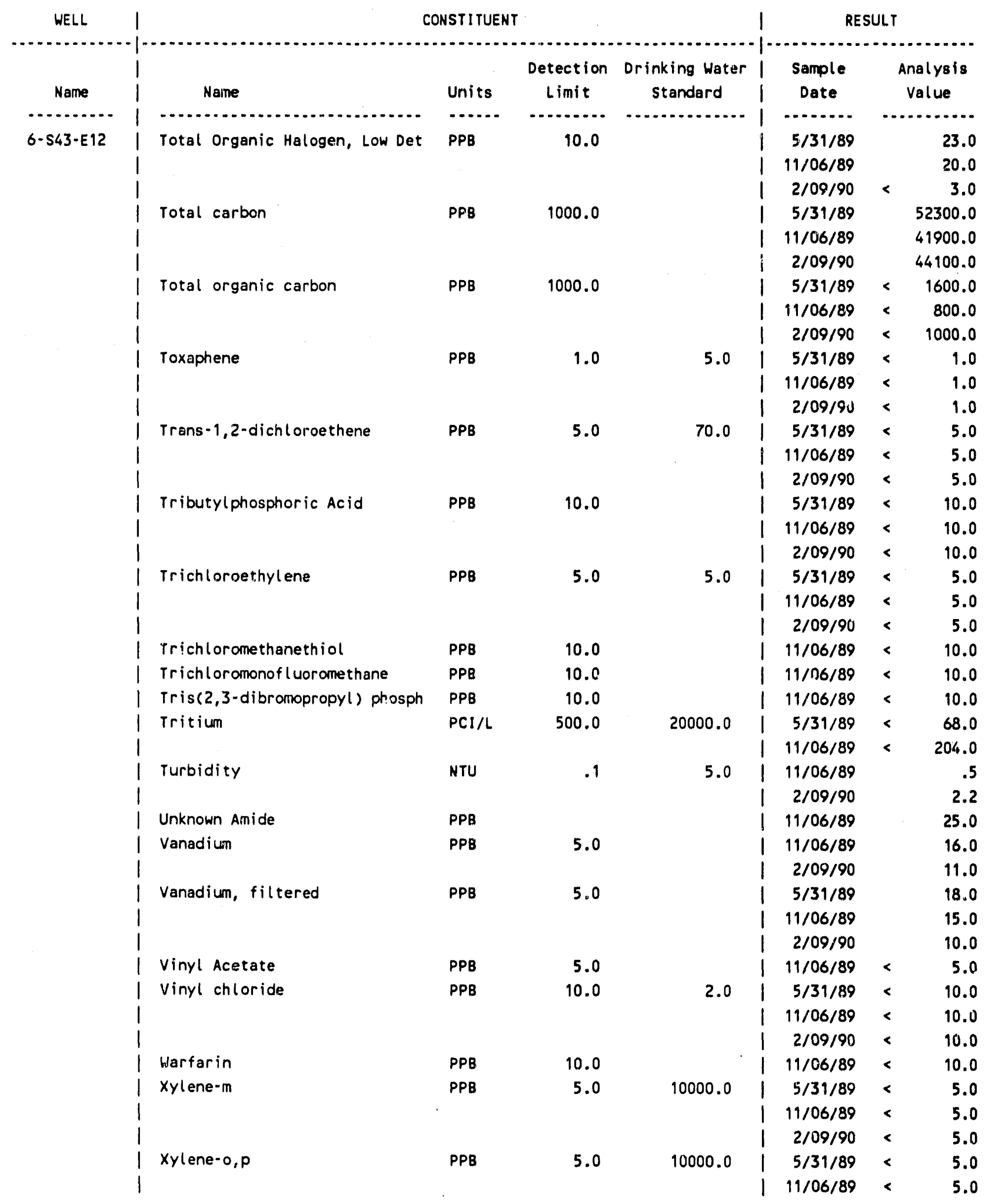




\section{WHC-MR. 0229}

$11 / 26 / 90$

Geosciences Group PARADOX Database

Well Result Report

Page 93

1100 Area Groundwater Monitoring

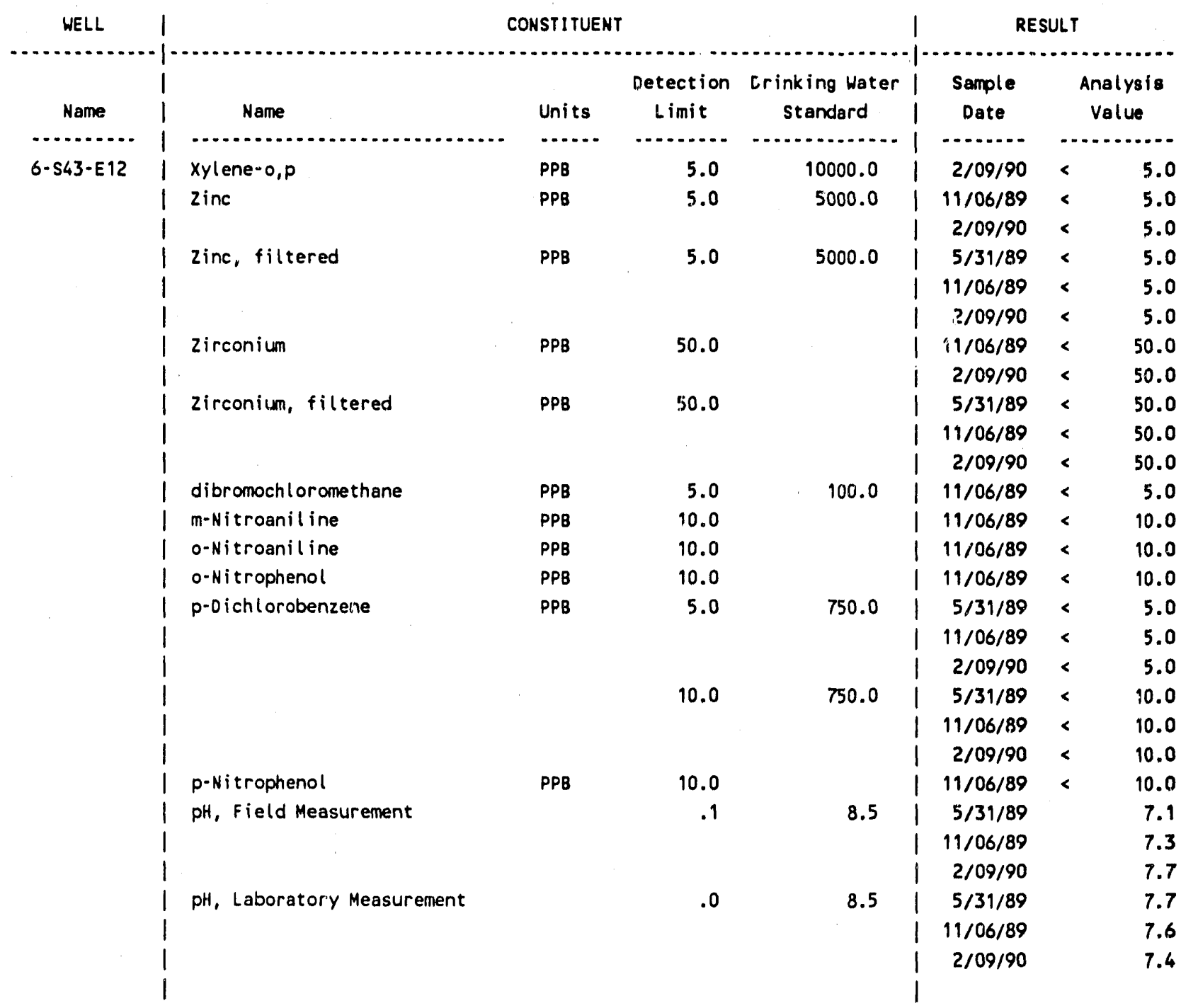



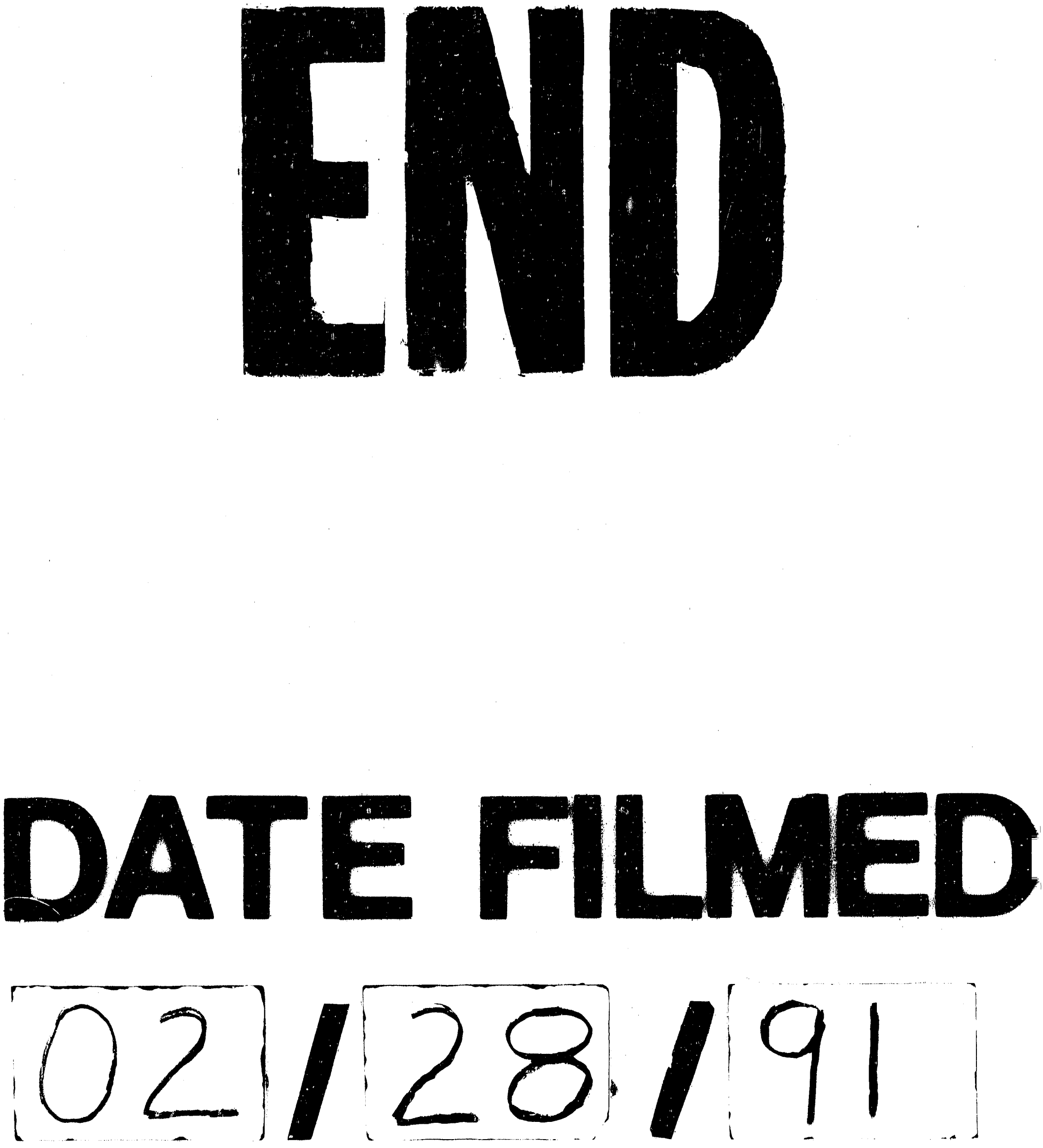
